\title{
Differing impacts of global and regional responses on SARS-CoV-2 transmission cluster dynamics
}

\author{
Brittany Rife Magalis, ${ }^{1,2 *}$ Andrea Ramirez-Mata, ${ }^{1,2}$ Anna Zhukova, ${ }^{3}$ Carla \\ Mavian, ${ }^{1,2}$ Simone Marini, ${ }^{2,4}$ Frederic Lemoine, ${ }^{3}$ Mattia Prosperi, ${ }^{4}$ Olivier \\ Gascuel, ${ }^{3}$ Marco Salemi ${ }^{1,2}$ \\ ${ }^{1}$ Department of Pathology, Immunology, and Laboratory Medicine, University of Florida, \\ Gainesville, Florida, 32610, USA \\ ${ }^{2}$ Emerging Pathogens Institute, University of Florida, Gainesville, Florida, 32610, USA \\ ${ }^{3}$ Department of Computational Biology, Institut Pasteur, Paris, 75015, France \\ ${ }^{4}$ Department of Epidemiology, University of Florida, Gainesville, Florida, 32610, USA \\ *To whom correspondence should be addressed; E-mail: salemi@ pathology.ufl.edu or \\ brittany.rife@epi.ufl.edu.
}

Although the global response to COVID-19 has not been entirely unified, the opportunity arises to assess the impact of regional public health interventions and to classify strategies according to their outcome. Analysis of genetic sequence data gathered over the course of the pandemic allows us to link the dynamics associated with networks of connected individuals with specific interventions. In this study, clusters of transmission were inferred from a phylogenetic tree representing the relationships of patient sequences sampled from December 30, 2019 to April 17, 2020. Metadata comprising sampling time and location were used to define the global behavior of transmission over this earlier sampling period, but also the involvement of individual regions in transmission cluster dynamics. Results demonstrate a positive impact of international travel restrictions and nationwide lockdowns on global cluster dynam- 


\section{ics. However, residual, localized clusters displayed a wide range of estimated initial secondary infection rates, for which uniform public health interventions are unlikely to have sustainable effects. Our findings highlight the presence of so-called "super-spreaders", with the propensity to infect a larger-than- average number of people, in countries, such as the USA, for which additional mitigation efforts targeting events surrounding this type of spread are urgently needed to curb further dissemination of SARS-CoV-2.}

Since its emergence from Wuhan, Hubei Province, China, in 2019 and its established humanto-human transmission, government and local bodies have been working to control the spread of coronavirus disease (COVID-19). Severe acute respiratory syndrome coronavirus 2 (SARS$\mathrm{CoV}-2$ ), the pathogen responsible for this disease, is a single-stranded RNA virus that likely emerged through recombination events within animal reservoirs infected by different strains $(1,2)$. Since December 2019, its rapid spread throughout the world has already resulted in more than ten million cases and hundreds of thousands deaths, with no vaccine or specialized medication currently available.

Prior to COVID-19, the most recent global pandemic that presented a serious public health emergency was caused by influenza A H1N1 strain in 2009. H1N1's threat exposed vulnerable public health capacities at the global, national and local levels, which we are facing once again, such as limitations of scientific knowledge, dilemmas in decision making, and communication among experts, policymakers and the public (3). The failure of preventive measures can lead to outbreaks crossing borders and exceeding national capacities (4). The likelihood of worldwide spread for pathogens characterized by human-to-human transmission, such as H1N1 and SARS$\mathrm{CoV}-2$, is extremely high with today's globalized economy and ease of international travel and requires additional, concerted efforts of governments and public health institutions to prevent or contain outbreaks. As individual nations have responded in various ways, and at varying times 
throughout the pandemic, understanding the impact of these efforts on the global and regional transmission dynamics of the virus are imperative in defining a strategy to assess the current situation and prevent similar scenarios in the future.

Genomic data sampled from viral epidemics offer a unique opportunity to evaluate not only the evolution of the virus over time but also the changing viral population dynamics imprinted in the evolutionary history. These population dynamics, though often reflective of the neutral processes of evolution (5), can at times be traced to significant ecological and epidemiological events (6). Sample collection dates are critical in these connections, as when combined with the assumption of relatively stable mutation rates over time, allow for genetic differences to be rescaled as differences in time. The timing of population changes, inferred from genetic changes, can then be compared with external, contextual data (e.g., (7)) to investigate potential links between public health interventions (or other relevant events) and viral population growth and spread. Global analyses of viral spread using genomic data can reveal important information regarding the emergence of a novel virus, such as the phylogenetic analysis of H1N1 (8), which informed the community of the adaptive process of H1N1 from its original host (swine) to humans and its subsequent challenges in escape from the human immune system. Since then, large projects aimed at facilitating and optimizing the sharing of data and results for real-time projections of viral spread have helped in tracking SARS-CoV-2 global dissemination (e.g., $(9,10))$. Yet, given that viruses evolve at a relatively rapid rate, considering separate isolated geographic areas as separate epidemics may also be warranted when attempting to understand how regional efforts drive viral evolutionary and population dynamic patterns. A virus from country $\mathrm{X}$ that seeds infection in country $\mathrm{Y}$ (founder event) becomes, over time, genetically distinct from the strains circulating in the country of origin, even in the absence of selection, due to genetic drift. On the other hand, in the era of globalization, analyses on regional epidemics limited to regional data can miss out on critical travel-mediated variables, such as separate, 
independent introductions of the virus (11).

Viral genetic data are not only useful in reconstructing the evolutionary and demographic history of a viral epidemic but also in identifying putative direct transmission events when $a$ priori knowledge, or estimates, of the maximum genetic distance that separates linked individuals exists (e.g., (12)). Transmission clusters are of interest to public health, as they represent groups of individuals related by a common denominator, or risk factor, such as locality, social network structure, or other behavior (e.g., (13)); the connectedness of these individuals is reflected in closely related genetic sequences. With advanced efforts in SARS-CoV-2 genomic sequencing, phylogenetic tools can help to identify and characterize clusters of transmission, as well as regions involved in those clusters. Using such transmission cluster data, we propose that patterns in cluster formation, growth, and connectedness among otherwise separate geographical regions offer insight not only into global components of the public health response, but can also help in identifying clusters and regions for which a more specific strategy to control local spread is required.

\section{Results}

Cluster size and composition Using a large genomic dataset collected from the Global Initiative on Sharing All Influenza Data (GISAID) aggregation of SARS-CoV-2 data, we hypothesized the existence of a relationship between international travel restrictions and overall transmission cluster dynamics, as well as sufficient variability among clusters in both space and time that would reveal the impact of varied local public health interventions. Based on the CDC definition of molecular transmission clusters for HIV, well-supported clades of viral sequences (one sequence per patient) comprised of at least 5 individuals with similar genetic distances were considered in this study to qualify as putative transmission clusters (also used in (14) for SARS-CoV-2). The criterion for similar genetic distances within these clades was a median 
patristic distance (branch length separating sequences within the tree) of $<0.009 \%$ (see Supplementary Materials) in a maximum likelihood (ML) phylogenetic tree inferred from 11,069 SARS-CoV-2 genomic sequences. This distance, representing the median genetic difference between two sampled individuals is less than the median difference observed within a single individual $(0.014 \%)$ in the study by Shen et al. (2020) (15) on patients admitted with COVID-19 pneumonia. By only considering individuals that share a genetic distance less than would be expected during the evolution of the virus within a single host, we have greater confidence in infections separated by a short amount of time and thus an epidemiological linkage or connection; increasing genetic distance leads to increased uncertainty as to the relationship of individuals within a cluster and a greater likelihood of the inclusion of multiple risk factors. The majority of clusters identified using this method included $<25$ individuals, with one large outlier cluster of 185 individuals (184 from the USA and 1 from Denmark) (Figure 1A). In terms of country representation within transmission clusters, the majority of the identified clusters included viral sequences isolated from patients in $1-5$ countries, with the majority country in clusters comprised of only two countries representing 50-99\% of the sequences (Figure 1B). Clusters including 6-10 countries were more evenly distributed, while the majority country represented $70 \%$ of the sequences in the only cluster with strains from 11 countries. The results collectively indicate a significant role for travel in connecting several countries through putative direct transmission events, rather than isolated epidemics seeded by single introductions, consistent with previous studies $(16,17)$.

As with conventional epidemiological analyses, sampling bias can impact results and interpretation and has been inherent to SARS-CoV-2 sample collection throughout the pandemic (18). In our analysis, the number of clusters involving each of the countries with available sequence data as of April 24, 2020, were distributed similarly to the number of available genomes indicating, unsurprisingly, that the number of clusters detected for a particular country is limited 
by the number of available samples from that locale (Figure 2A). Hence, no direct comparison could be made regarding the number of clusters involving different countries. This was not the case, however, for the average size (number of persons) of clusters associated with each country, since large clusters were not always detected in countries with more samples available, such as the United Kingdom (UK) and United States of America (USA) (Figure 2B). In other words, despite potential lack of information from countries with reduced datasets, a global perspective on transmission cluster patterns related to cluster size might nevertheless be investigated. It is important to keep in mind that low sequence presence is not necessarily an indicator of low infection rates, as the fraction of total infected individuals that were sampled may, in fact, be high for these countries, resulting in a different type of regional sampling bias. For example, although as of April 24, over 3,000 sequences were submitted for the UK, this comprised $<25 \%$ of total infections, whereas Hong Kong reportedly submitted at least one genome for every confirmed case (Figure 2C). Given that individuals linked through transmission in a small amount of time share a small genetic difference, on which phylogenetic cluster inference relies, missed sampling can prevent the inclusion of individuals within a cluster. Hence, we anticipated that sampled individuals from countries with sequencing more representative of the infected population (i.e., genome per confirmed case value closer to 1) would be more likely to cluster, resulting in a larger fraction of clustered individuals. However, there was no clear relationship (linear regression $R^{2}<0.0084$ ) between the percentage of individuals within a country that are included in clusters and the number of retrieved genomes per confirmed cases (Figure 2C). This finding indicates that country-specific contributions to clustering were not biased toward countries with larger fractions of sampled individuals from the infected population. Therefore, sub-sampling as an effort to mitigate the effects of sampling bias at the country level, though often performed for phylogenetic analyses of viral geographical spread (19), was not deemed necessary for our study. On the other hand, it is important to notice that due to the lack of information on sam- 
pling strategies used to gather available sequence data, we could not rule out possible effects of selection bias (i.e., preferential sampling). For example, in certain countries for which representative sequencing was low but clustering rate was high, we cannot exclude that sampling efforts were focused on presumed contact networks in order to locate and quarantine infections.

Cluster origin and epidemiology In order to derive epidemiological information for each detected cluster, branch lengths within the tree were scaled in time by enforcing a molecular clock, which assumes the accumulation of substitutions has occurred at a constant rate over time. The time to the most recent common ancestor (TMRCA) of all sequence data was estimated to be December 6 [25 Nov - 10 Dec] 2019 (Figure S1, consistent with previous demographic modelbased estimate by Andersen et al. (2020) (20). The result was a good indication that date estimates for remaining internal nodes within the tree were also reliable.

For the majority of the transmission clusters detected in the tree, TMRCAs (i.e., a cluster's temporal origin) dated back prior to Feb 20, 2020, with a peak observed between the first week of February until the first week of March (Figure 3A). These results were robust to genetic distance thresholds of $0.006 \%$ and $0.013 \%$ (Figure S2), the latter value representing the 2.5 th percentile of tree-wide distances and maximum diversity observed in Shen et al. (2020) (15), as described above. Following this time, a sharp decline in number of newly formed clusters was evident. Furthermore, despite differing patterns in the number and size of clusters across individual countries, the global peak in clusters size coincided with the peak in number of new clusters, around the end of February, as did the number of countries represented in each cluster (Figure 3A). The overlapping peaks in cluster number and size, as well as number of countries/cluster in time, suggest a common underlying factor responsible for the decrease in the rate of cluster formation, growth, and connectivity at the global level. Thus, it appeared reasonable to hypothesize that efforts to reduce international travel at the beginning of the epidemic 
played a role.

Data on reported travel restrictions (Tables S1 and S2) were obtained from various sources (see Supplementary Materials), and the cumulative number of reports involving all international travel, as well as those specifically involving China, were plotted over time to provide context into travel-related events that would potentially result in the global cluster behavior described above. The early, elevated rate of accumulation of travel bans, largely comprised of restriction on immigration from China began to slow on February 15 (slowed accumulation for Chinaspecific bans on the 6th) (Figure 3B). Accumulation in the number of restrictions remained low until the onset of nationwide lockdowns (first reported March 08), at which time the steep decline in overall cluster TMRCA, size, and geographical range began. These results strongly suggest that reduced international travel slowed the formation, growth, and connectivity of transmission clusters, whereas more local interventions were necessary, and more effective, in preventing local virus spread.

The median lifespan, or duration, of a transmission cluster was estimated to be approximately 2 weeks, with the largest cluster extending for over six weeks (Figure 4A). Such a short duration time (on the order of the longer end of the incubation period (21)), combined with average cluster sizes of up to 25 sampled individuals, is indicative of SARS-CoV-2 rapid transmission (22). The expected number of secondary cases directly generated by a primary case in the population, otherwise known as the basic reproductive number $\left(R_{0}\right)$, was calculated as a function of early changes in the estimated viral effective population size (23) and a normally distributed infectious period of approximately $2-8$ days). $R_{0}$ for the entire pandemic was estimated at 5.65 [95\% credible interval (CI): 4.37-6.68], consistent with previously reported $R_{0}$ s by Shen et al. (2020) (15) and Tang et al. (2020) (24), as well as other published (but not peer-reviewed) estimates reviewed in Liu et al. (2020) (22). However, in contrast to previous epidemiological studies, we utilized phylogenetic methods at an increased resolution to 
estimate the transmission potential for individual clusters, which we have already shown can vary in size and composition over time. In the majority of clusters, $R_{0}$ ranged from $<1$ to 2.32 , though $R_{0}$ values of up to 12 were reported (Figure 4B), indicative of clusters formed through "super-spreading" events (SSEs), or cases of larger-than-average transmissibility (25). While it is important to note that accuracy of $R_{0}$ estimation is reduced for outbreaks characterized by true $R_{0} \geq 5$ (23), clusters representing increased secondary infection rates as compared to the majority population are of importance in the control of infection. As $R_{0}>1$ is indicative of sustainable transmission and vice versa, we investigated the timing and duration of both $\operatorname{low}(<1)$ and high $(>1)-R_{0}$ clusters. High- $R_{0}$ clusters were more frequently observed between February and early March (Figure 4C), consistent with the temporal peak in global cluster size. There was no relationship between $R_{0}$ and duration $\left(R^{2}<3.69 E-06\right)$, suggesting clusters with $R_{0}<1$ were still sustained for various lengths of time. Although seemingly counter-intuitive, this finding can be explained by dynamic transmission patterns, such as a change in the contact network, or even a later introduction of unsampled super-spreaders. As the $R_{0}$ calculation is derived from early estimates of the viral effective population size, this value does not depict the full transmission potential of the group of individuals within the cluster.

Our results point to a drastic reduction in cluster formation, growth, and connectivity following the first week of March. Yet, clusters with $R_{0}>2$ (earlier estimates of SARS-CoV-2 $R_{0}$ ) were observed during this time (Figure 4C). We next sought to investigate which countries were involved in the few, albeit seemingly rapidly spreading, clusters that formed following the onset of lockdown measures (e.g., social distancing). $R_{0}$ values across clusters for each country were averaged after being scaled based on the percentage of sequences belonging to that country, resulting in a weighted mean $R_{0}$ (Figure 5A). Belgium, Luxembourg, and the UK and USA (alphabetical order only) were estimated to have a weighted mean $R_{0}>2$ in March, whereas Australia, Iceland, and the Netherlands were approximately 1 or less $\left(R_{0}<1.1,1.2\right.$, and 1 , 
respectively) (Figure 5A). In line with global reduction in connectivity, clusters formed after March 08 consisted of 2 countries or less Figure 5B. The USA formed two clusters comprised of only US individuals, both with $R_{0}>2$, one of which was estimated as the highest $R_{0}$ value ( $>$ 10, CI: 7.57-11.94) of all clusters at this time. The three clusters following in ranking comprised either Belgium alone (2) or Belgium and neighboring Luxembourg (2), with $R 0>4$. All of these clusters exhibited evidence of sustained transmission, with a duration of greater than 2 weeks, though it is important to note that the addition of more up-to-date sequences may extend duration times for clusters initiated during this time period. The UK formed three separate clusters - one isolated, one with primarily UK individuals, and one with primarily Australian individuals. The two clusters comprised of majority UK sequences were both estimated to have $R 0>2$, whereas the cluster with primarily Australian sequences was characterized as $R 0<1$. Similarly, the second Australian cluster (Australian sequences only) was also characterized as having a relatively low $R_{0}$ of $<1.3$ (CI:1.20-1.32). Results suggest that the virus was already spreading rapidly in the USA, Belgium and Luxembourg, and the UK at the time of implementation of regional mobility restriction efforts and that, despite efforts to restrict international travel, the UK and Australia maintained travel sufficient to sustain inter-regional transmission.

Recent evidence implicating a mutation in residue 614 of the spike protein of SARS-CoV-2 in increased infectivity (26) and higher mortality (27) offered a possible explanation for increased transmission potential of certain clusters identified in this study. However, despite the clear advantages at the cellular level of glycine in place of aspartic acid at this site, there was no relationship between prevalence of glycine (\% of individual sequences) within individual transmission clusters and $R_{0}$ (linear regression $\left.R^{2}<0.00011\right)$ or cluster size $\left(R^{2}<0.0083\right)$ (Figure S3), though generation time was not explored. 


\section{Discussion}

Unlike conventional epidemiological surveillance data, viral genetic data can be used to link infected individuals involved in direct transmission events even when the contact structure is unknown. Identification of these putative transmission clusters, coupled with phylogenetic inference of cluster dynamics and relevant epidemiological parameters, has provided evidence of a global pattern in cluster formation, growth, connectivity, as well as transmission potential over time. The slow rise and subsequent rapid fall in the number and size of forming clusters over the period of December 30 to April 20, 2020, may have been the results of a change in global transmission patterns and/or a rate of sampling that did not adequately capture, as recently reported (28), the increased rate in the number of infected individuals. According to the latter scenario, missed sampling of individuals involved in a transmission cluster can prevent the detection of links via genetic data, or even conventional surveillance data. However, the similar temporal pattern observed for the number of countries connected by individual clusters also suggests a relationship between cluster formation, growth, and connectivity and that an outside force was responsible for changing global transmission patterns, rather than problematic sampling over time.

When placed in the context of the timing and accumulation of public health interventions, the data provide evidence supporting the benefits of both global and regional response efforts. Given an incubation period extending to up to 18 days within an individual (29), it is plausible that specific restriction on international travel involving China, peaking on February 06, could have resulted in the slowed rate of cluster activity beginning the first week of February. It is also possible that the rapid increase in overall international travel restrictions, peaking around February 15, was partially, if not equally, responsible. Whereas additional modeling using recorded travel data would be highly beneficial in teasing apart the effects of international and 
China-specific travel restrictions on transmission characteristics, the drastic halt in cluster activity beginning the first week of March directly coincides with the date of onset of nationwide lockdowns. This particular finding speaks to the effectiveness of additional non-pharmaceutical measures taken at the regional level to curb the spread of infection, consistent with previous reports $(30,31)$.

Whereas a global reduction in cluster activity in response to efforts to reduce mobility might be expected given the known role of travel in pathogen spread (32), we anticipated cluster- and region-specific variation in transmission characteristics, consistent with known difficulties in controlling regional epidemics. Using viral sequence data and the phylogenetic relationships among sampled individuals, particularly for an exponentially growing epidemic of limited data availability (23), we can model relevant epidemiological parameters of interest used in determining the transmission potential for clusters involving individual countries. It is important to keep in mind that the results of any phylogenetic study that are dependent on a single tree assume that that a tree best describes the underlying phylogenetic relationships. While it is often best to summarize results across a sample of similarly plausible trees using Bayesian methods $(33,34)$, Bayesian tree reconstruction methods are highly parametric and have difficulty converging on a reliable distribution of trees and related evolutionary parameters for datasets as large as that of SARS-CoV-2. For this reason, we only focused this study on portions of the maximum likelihood tree that were considered to be well supported. Similarly, invaluable methods exist for the detection and epidemiological characterization of transmission clusters within the Bayesian framework, such as the multi-state birth death model (bdmm) (35); however, even the bdmm is limited to less than 1000 sequences (unpublished work by Scire et al. ( (36)). While sub-sampling strategies used to reduce dataset size and potential sampling biases are widely appreciated in the field of phylogenetic epidemiology (e.g, (19)), they inherently result in loss of smaller clusters, which play an important role in assessing the effect of social distancing in- 
terventions. As our study is based on hypotheses regarding not only cluster characteristics but also cluster size distribution, a skew towards detection of larger clusters as a result of this loss of information was undesirable. Post hoc transmission characterization of individual clusters using a Bayesian framework for population dynamics estimates that has demonstrated accuracy for low-signal sequence data (i.e., small clusters) (23) was thus ideal for an in-depth investigation of transmission cluster dynamics at the global and regional scales. Using this method, four countries (the USA, Belgium and neighboring Luxembourg, and the UK) were identified as harboring isolated clusters with elevated transmission potential following the global initiation of nationwide lockdowns, potentially fostered by super-spreaders. Federal policies regarding lockdowns were put into place for Belgium, Luxembourg, and the UK beginning mid-March, which were likely necessary in absolving highly active clusters such as those observed in this study; though federal guidelines were issued at a similar time in the USA (March 16th), mandatory US policy regarding lockdowns was not put into motion, allowing individual states to adopt their own policies at different times. Depending on the location of transmission clusters within the USA, delayed lockdowns could have resulted in continued rapid transmission of the virus. It is also important to keep in mind that at the time of this study, a dramatic rebound was being observed in the number of detected daily cases in the USA, whereas the UK, Belgium and Luxembourg were demonstrating a consistent decline with occasional peaks (9). Moreover, the epidemic at the time was exponentially spreading in Brazil and India, as well as steadily in Russia - all countries that were not captured by our analysis based on sequence data up to the last week in April, 2020. In this context, our results emphasize the importance of additional country-specific transmission cluster analysis for data collected more recently than April 20.

It would be reasonable to suspect that the identification of the USA, Belgium, Luxembourg, and the UK as problematic countries was aided by the availability of sequence data from these locations (i.e., attributed to a sufficient number of individuals and genetic diversity to classify 
and characterize corresponding clusters), which at first points to a potential problem with sampling bias. Although these countries were indeed at the high end of the spectrum in terms of available sequence data, our analyses relied on averaged values across clusters, which we show is unrelated to genome availability, unlike number of clusters per country. These countries exhibited a wide range of clustering frequency (20-60\% of individuals) with no relation to sampling representation (fraction of the infected population sampled), which varied from 1-30\%, indicating with a high degree of confidence, that they were not identified as a result of sampling bias. Moreover, countries (Iceland, Australia, and the Netherlands) identified as having low transmission potential after March 08 ( $R_{0}$ less than or approximately 1$)$ had a comparable number of available genomes within the distribution, lending support to the conclusion. This is not to say that selection bias, a form of sampling bias, may not be associated with the results. For example, Iceland's low $R_{0}$ during this time may not be surprising, given that the country's genetic powerhouse, deCODE, began screening high-risk (symptomatic) individuals and those returning, or in contact with an individual, from high-risk countries as early as January 31 (37). Therefore, while in our study, concerns for the sampling representation of the overall infected population is negligible, more sophisticated quantitative measures to assess the impact of sampling biases, specifically selection bias, will be necessary in future investigations.

In summary, we propose that phylogenetic identification and characterization of transmission clusters using the vast resources of viral genomic data currently available can provide both global and regional perspectives on viral spread. When collected early in the course of an epidemic, as was the the case for the SARS-CoV-2, this approach may help to pinpoint locations for which increased efforts at the level of local government might be necessary to mitigate growth on a pandemic scale. At the time of the submission of these results, relaxation of these efforts was on the rise, particularly in the USA. The detection of isolated transmission clusters with elevated transmission potential in March, despite the rapid decline in global patterns of clus- 
ter activity, points to an important role played by super-spreaders in the current pandemic that likely pose a threat to relaxation (38) unless measures are taken to quickly recognize and predict these events. A better understanding of the underlying risk factors associated with related super-spreading events, including host, environmental, and behavioral factors (39), is necessary for targeting efforts aimed in avoiding recurrent rebounds in epidemic waves. Given the increased efforts in testing and sampling in the USA, as well as other countries, transmission cluster dynamic inference can help to identify these events and underlying risk networks for more precise intervention strategies.

\section{Acknowledgements}

We would also like to acknowledge the health workers and researchers who generated the data,without whom this work would not have been possible. Funding for this work was provided by the National Science Foundation (DEB 2028221) and National Institutes of Health (R21AI138815).

\section{Author contributions statement}

M.S., M.P., and B.R.M. conceived of the analyses, S.M. and C.M. retrieved the data, A.Z. and F.L. used their expertise (with guidance from O.G.) in sequence data quality control and tree reconstruction to produce the trees, M.P. used his expertise in Phylopart to identify transmission clusters, B.R.M. analyzed the clusters and prepared the manuscript, A.R. monitored and gathered data regarding region-specific public health interventions, and all authors helped to craft the discussion and review the the manuscript. The authors report no competing interests at the time of manuscript preparation and submission. All data is available in the manuscript or the supplementary materials. 


\section{References}

1. Li, X. et al. Emergence of sars-cov-2 through recombination and strong purifying selection. Science Advances (2020). URL https://advances. sciencemag.org/content/early/2020/05/28/sciadv.abb9153.

https://advances.sciencemag.org/content/early/2020/05/28/ sciadv.abb9153. full.pdf.

2. Xiao, K. et al. Isolation of SARS-CoV-2-related coronavirus from Malayan pangolins. Nature (2020).

3. Fineberg, H. V. Pandemic preparedness and response-lessons from the H1N1 influenza of 2009. N. Engl. J. Med. 370, 1335-1342 (2014).

4. Moon, S. et al. Will Ebola change the game? Ten essential reforms before the next pandemic. The report of the Harvard-LSHTM Independent Panel on the Global Response to Ebola. Lancet 386, 2204-2221 (2015).

5. Frost, S. D. W., Magalis, B. R. \& Kosakovsky Pond, S. L. Neutral Theory and Rapidly Evolving Viral Pathogens. Mol. Biol. Evol. 35, 1348-1354 (2018).

6. Rife, B. D. et al. Phylodynamic applications in 21 st century global infectious disease research. Glob Health Res Policy 2, 13 (2017).

7. Mavian, C. et al. Emergence of recombinant Mayaro virus strains from the Amazon basin. Sci Rep 7, 8718 (2017).

8. Su, Y. C. F. et al. Phylodynamics of H1N1/2009 influenza reveals the transition from host adaptation to immune-driven selection. Nat Commun 6, 7952 (2015). 
9. Dong, E., Du, H. \& Gardner, L. An interactive web-based dashboard to track COVID-19 in real time. Lancet Infect Dis 20, 533-534 (2020).

10. Shu, Y. \& McCauley, J. GISAID: Global initiative on sharing all influenza data - from vision to reality. Euro Surveill. 22 (2017).

11. Rhee, S.-Y. et al. National and International Dimensions of Human Immunodeficiency Virus-1 Sequence Clusters in a Northern California Clinical Cohort. Open Forum Infectious Diseases 6 (2019). URL https://doi.org/10.1093/ofid/ ofz135. Ofz135, https://academic.oup.com/ofid/article-pdf/6/4/ ofz135/28530447/ofz135.pdf.

12. Smith, D. M. et al. A public health model for the molecular surveillance of hiv transmission in san diego, california. AIDS (London, England) 23, 225-232 (2009). URL https: //pubmed.ncbi.nlm.nih.gov/19098493. 19098493[pmid].

13. Aldous, J. L. et al. Characterizing hiv transmission networks across the united states. Clinical infectious diseases : an official publication of the Infectious Diseases Society of America 55, 1135-1143 (2012). URL https : / /pubmed.ncbi.nlm.nih.gov/ $22784872.22784872[$ pmid].

14. Furuse, T. et al. Clusters of coronavirus disease in communities, Japan, January-April 2020. Emerg Infect Dis (2020). URL https://doi.org/10.3201/eid2609. 202272 .

15. Shen, Z. et al. Genomic diversity of sars-cov-2 in coronavirus disease 2019 patients. Clinical infectious diseases : an official publication of the Infectious Diseases Society of America ciaa203 (2020). URL https://pubmed.ncbi.nlm.nih.gov/32129843. 32129843 [pmid]. 
16. Deng, X. et al. Genomic surveillance reveals multiple introductions of sars-cov2 into northern california. Science 369, 582-587 (2020). URL https:// science.sciencemag.org/content/369/6503/582. https://science. sciencemag.org/content/369/6503/582.full.pdf.

17. Gonzalez-Reiche, A. S. et al. Introductions and early spread of sars-cov-2 in the new york city area. Science 369, 297-301 (2020). URL https:// science.sciencemag.org/content/369/6501/297. https://science. sciencemag.org/content/369/6501/297.full.pdf.

18. Mavian, C., Marini, S., Prosperi, M. \& Salemi, M. A Snapshot of SARS-CoV-2 Genome Availability up to April 2020 and its Implications: Data Analysis. JMIR Public Health Surveill 6, e19170 (2020).

19. Hong, S. L. et al. In search of covariates of hiv-1 subtype b spread in the united states-a cautionary tale of large-scale bayesian phylogeography. Viruses 12 (2020).

20. Andersen, K. G., Rambaut, A., Lipkin, W. I., Holmes, E. C. \& Garry, R. F. The proximal origin of SARS-CoV-2. Nat. Med. 26, 450-452 (2020).

21. The incubation period of coronavirus disease 2019 (covid-19) from publicly reported confirmed cases: Estimation and application. Annals of Internal Medicine 172, 577582 (2020). URL https://doi.org/10.7326/M20-0504. PMID: 32150748, https://doi.org/10.7326/M20-0504.

22. Liu, Y., Gayle, A. A., Wilder-Smith, A. \& Rockl?v, J. The reproductive number of COVID19 is higher compared to SARS coronavirus. J Travel Med 27 (2020). 
23. Volz, E. M. \& Didelot, X. Modeling the Growth and Decline of Pathogen Effective Population Size Provides Insight into Epidemic Dynamics and Drivers of Antimicrobial Resistance. Syst. Biol. 67, 719-728 (2018).

24. Tang, B. et al. Estimation of the Transmission Risk of the 2019-nCoV and Its Implication for Public Health Interventions. J Clin Med 9 (2020).

25. Cave, E. Covid-19 super-spreaders: Definitional quandaries and implications. Asian bioethics review 1-8 (2020). URL https://pubmed.ncbi.nlm.nih.gov/ 32427202.32427202 [pmid].

26. Zhang, L. et al. The d614g mutation in the sars-cov-2 spike protein reduces s 1 shedding and increases infectivity. bioRxiv 2020.06.12.148726 (2020). URL http: / / biorxiv . org/content/early/2020/06/12/2020.06.12.148726.abstract.

27. Becerra-Flores, M. \& Cardozo, T. Sars-cov-2 viral spike g614 mutation exhibits higher case fatality rate. International Journal of Clinical Practice n/a, e13525. URL https: //onlinelibrary.wiley.com/doi/abs/10.1111/ijcp.13525. https: //onlinelibrary.wiley.com/doi/pdf/10.1111/ijcp.13525.

28. FU, X. Global analysis of daily new covid-19 cases reveals many static-phase countries including us and uk potentially with unstoppable epidemics. medRxiv 2020.05.08.20095356 (2020). URL http://medrxiv.org/content/early/2020/05/29/2020. 05.08 .20095356$. abstract.

29. Verity, R. et al. Estimates of the severity of coronavirus disease 2019: a model-based analysis. The Lancet Infectious Diseases 20, 669-677 (2020). URL https://doi . org/10.1016/S1473-3099(20)30243-7. 
30. Alfano, V. \& Ercolano, S. The efficacy of lockdown against covid-19: A cross-country panel analysis. Applied health economics and health policy 1-9 (2020). URL https: //pubmed.ncbi.nlm.nih.gov/32495067. 32495067[pmid].

31. Prem, K. et al. The effect of control strategies to reduce social mixing on outcomes of the covid-19 epidemic in wuhan, china: a modelling study. The Lancet Public Health 5, e261e270 (2020). URL https: / / doi .org/10.1016/s2468-2667 (20) 30073-6.

32. Findlater, A. \& Bogoch, I. I. Human mobility and the global spread of infectious diseases: A focus on air travel. Trends in Parasitology 34, 772-783 (2018). URL https : / / doi . org/10.1016/j.pt.2018.07.004.

33. Huelsenbeck, J. P. \& Ronquist, F. MRBAYES: Bayesian inference of phylogenetic trees. Bioinformatics 17, 754-755 (2001). URL https://doi.org/ 10.1093/bioinformatics/17.8.754. https://academic.oup.com/ bioinformatics/article-pdf/17/8/754/8201380/170754.pdf.

34. Drummond, A. J., Nicholls, G. K., Rodrigo, A. G. \& Solomon, W. Estimating mutation parameters, population history and genealogy simultaneously from temporally spaced sequence data. Genetics 161, 1307-20 (2002).

35. Barido-Sottani, J., Vaughan, T. G. \& Tanja, S. Detection of hiv transmission clusters from phylogenetic trees using a multi-state birth-death model. J. R. Soc. Interface 15 (2018). URL http://doi.org/10.1098/rsif.2018.0512.

36. Scire, J., Barido-Sottani, J., Kühnert, D., Vaughan, T. G. \& Stadler, T. Improved multi-type birth-death phylodynamic inference in beast 2 . bioRxiv (2020). URL https:// www.biorxiv.org/content/early/2020/01/06/2020.01.06.895532. 
https://www.biorxiv.org/content/early/2020/01/06/2020.01.06. 895532.full.pdf.

37. Gudbjartsson, D. F. et al. Spread of sars-cov-2 in the icelandic population. New England Journal of Medicine 382, 2302-2315 (2020). URL https://doi.org/10 . 1056 / NEJMoa2006100. https://doi .org/10.1056/NEJMoa2006100.

38. Riou, J. \& Althaus, C. L. Pattern of early human-to-human transmission of wuhan 2019 novel coronavirus (2019-ncov), december 2019 to january 2020. Euro surveillance : bulletin Europeen sur les maladies transmissibles = European communicable disease bulletin 25, 2000058 (2020). URL https://pubmed.ncbi.nlm.nih.gov/32019669. 32019669 [pmid].

39. Frieden, T. \& Lee, C. Identifying and interrupting superspreading events-implications for control of severe acute respiratory syndrome coronavirus 2. Emerging Infectious Disease journal 26, 1059 (2020). URL https : / / doi.org/10.3201/eid2606.200495.

40. Lemoine, F., Blassel, L., Voznica, J. \& Gascuel, O. Covid-align: Accurate online alignment of hcov-19 genomes using a profile hmm (2020). URL https : / / doi . org / 10 . 1101 / 2020.05 .25 .114884$.

41. Kozlov, A. M., Darriba, D., Flouri, T., Morel, B. \& Stamatakis, A. RAxML-NG: a fast, scalable and user-friendly tool for maximum likelihood phylogenetic inference. Bioinformatics 35, 4453-4455 (2019). URL https://doi.org/10.1093/bioinformatics/ btz305. https://academic.oup.com/bioinformatics/article-pdf/ $35 / 21 / 4453 / 30330793 /$ btz305.pdf.

42. Lefort, V., Desper, R. \& Gascuel, O. Fastme 2.0: A comprehensive, accurate, and fast distance-based phylogeny inference program. Molecular biology and evolution 
32, 2798-2800 (2015). URL https: / /pubmed.ncbi.nlm.nih.gov/26130081. $26130081[\mathrm{pmid}]$.

43. Anisimova, M. \& Gascuel, O. Approximate likelihood-ratio test for branches: A fast, accurate, and powerful alternative. Syst. Biol. 55, 539-552 (2006).

44. Minh, B. Q. et al. IQ-TREE 2: New Models and Efficient Methods for Phylogenetic Inference in the Genomic Era. Mol. Biol. Evol. 37, 1530-1534 (2020).

45. To, T. H., Jung, M., Lycett, S. \& Gascuel, O. Fast Dating Using Least-Squares Criteria and Algorithms. Syst. Biol. 65, 82-97 (2016).

46. Prosperi, M. C. F. et al. A novel methodology for large-scale phylogeny partition. Nature communications 2, 321-321 (2011). URL https : / / pubmed.ncbi.nlm.nih.gov/ $21610724.21610724[\mathrm{pmid}]$.

47. R Core Team. R: A Language and Environment for Statistical Computing. R Foundation for Statistical Computing, Vienna, Austria (2017). URL https : / /www.R-project . org/.
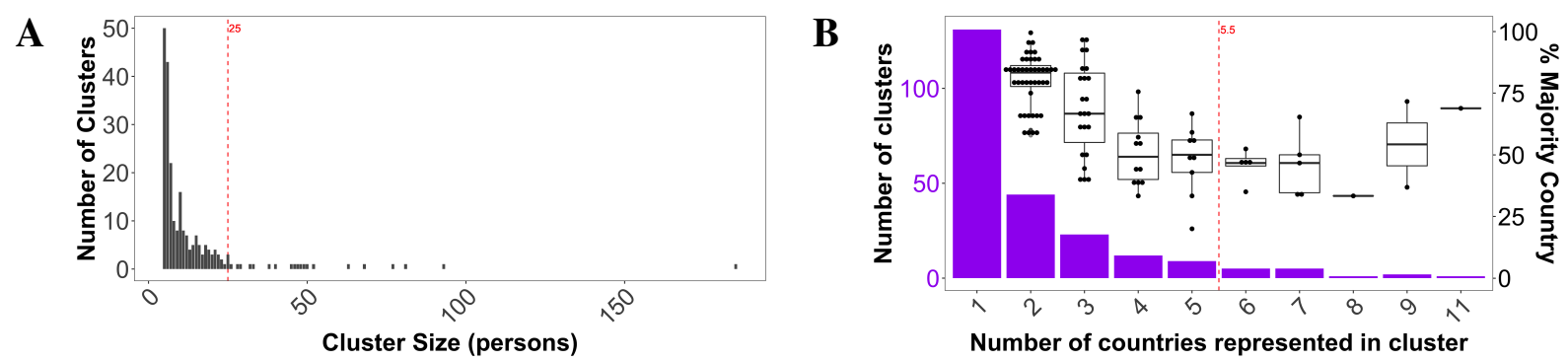

Figure 1: Transmission cluster distributions against cluster size (A) and number of countries represented in each cluster (B). Percentage of sequences comprising the majority represented country has also been plotted (box plots) in (B). Red, dashed lines indicate median values. 
A

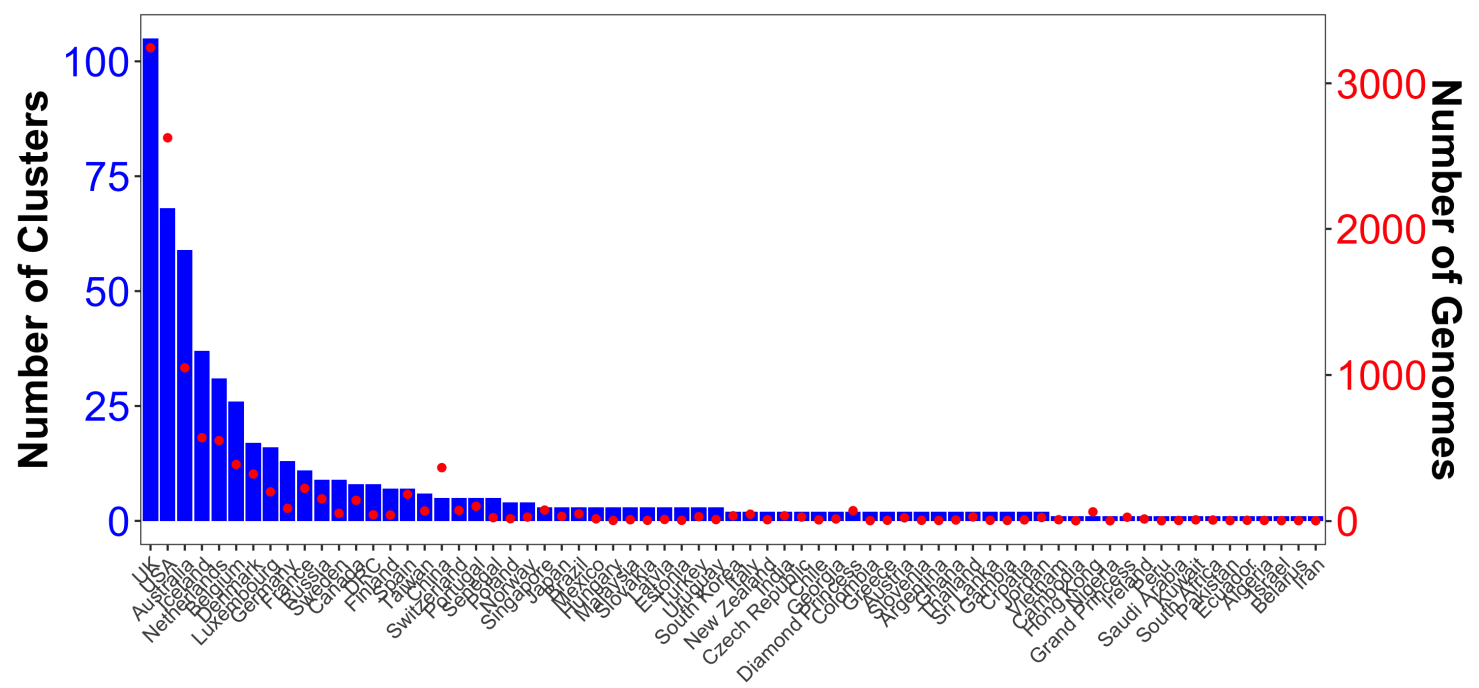

B

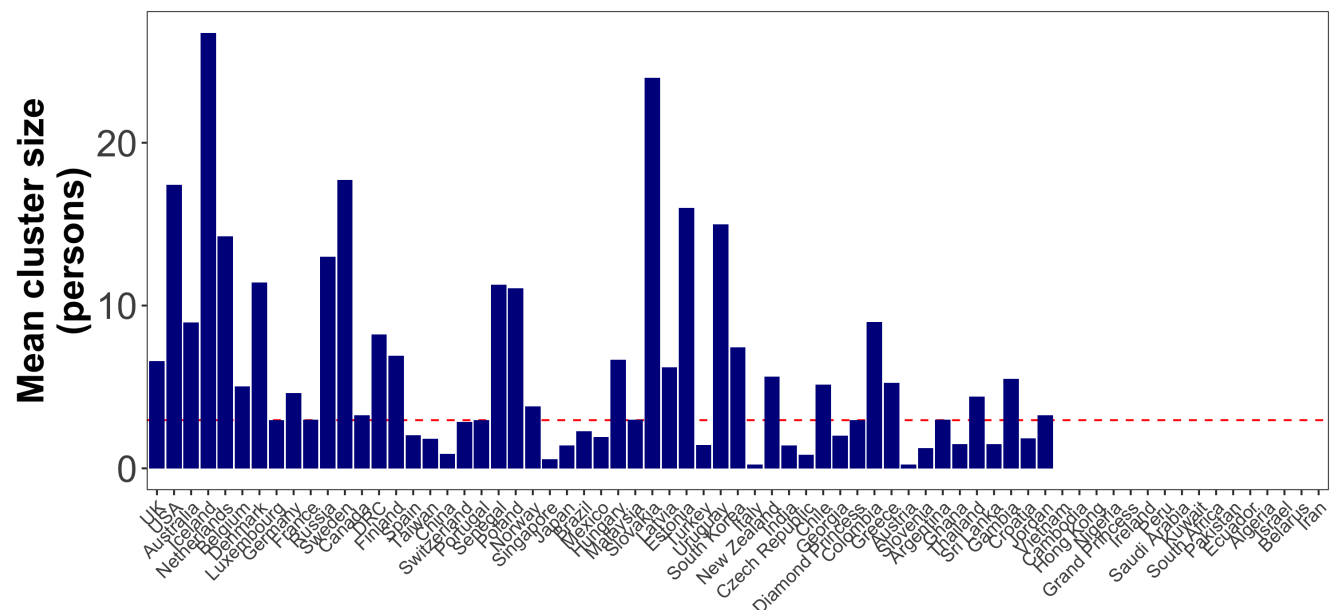

C

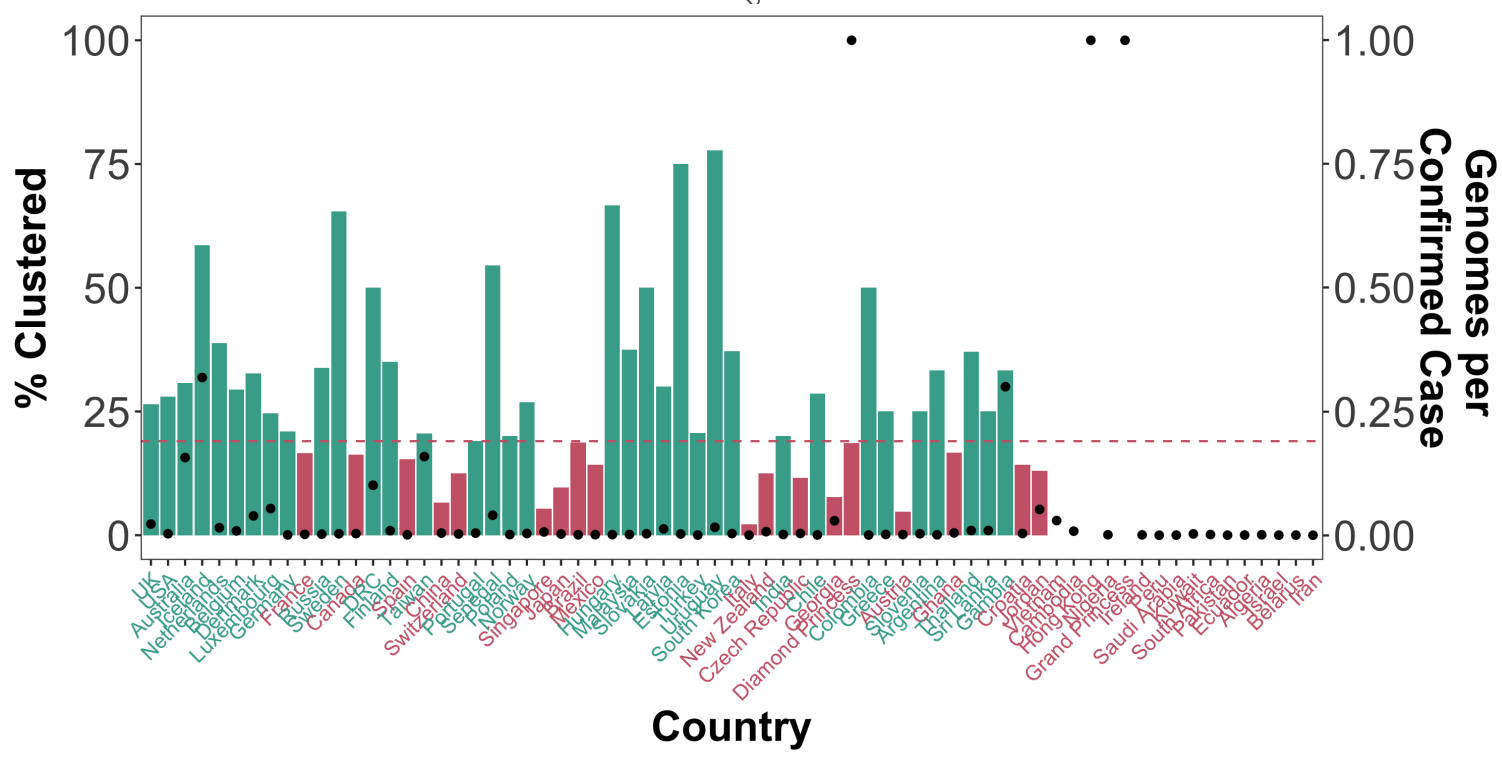

Figure 2: Transmission cluster characteristics for each country involved. Number of clusters (A), mean cluster size (B), and percentage of individuals that 2 Bister (C) are plotted for each country, as well as the number of available genomes (A) and genomes per confirmed case reports (C) for comparison. 
A

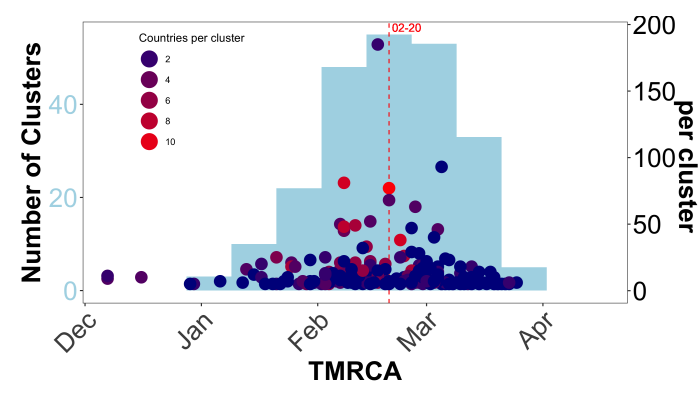

B

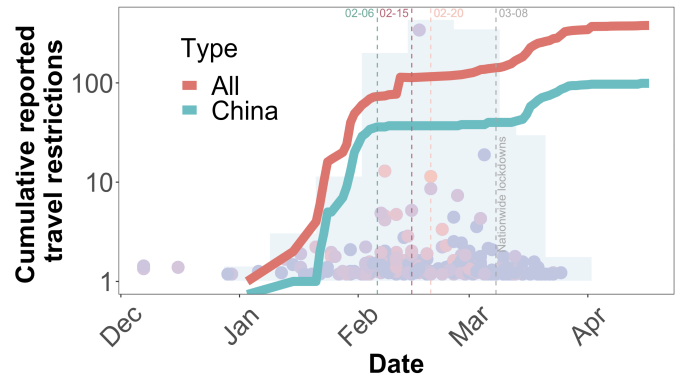

Figure 3: Timing of transmission cluster characteristics. (A) Number of clusters (light blue) sharing a similar temporal origin, as inferred from the time to the most recent common ancestor(TMRCA) of corresponding cluster sequences. Dots correspond to the size (number of individuals) within the corresponding clusters at individual time points. Dots are colored according to the number of countries represented in each cluster. (B) Number of cumulative reported international (red) and China (teal) travel restrictions over time superimposed onto (A), ending in the first reported case of local travel restrictions (grey) for visual clarity. Red, dashed lines indicated median values. 
A

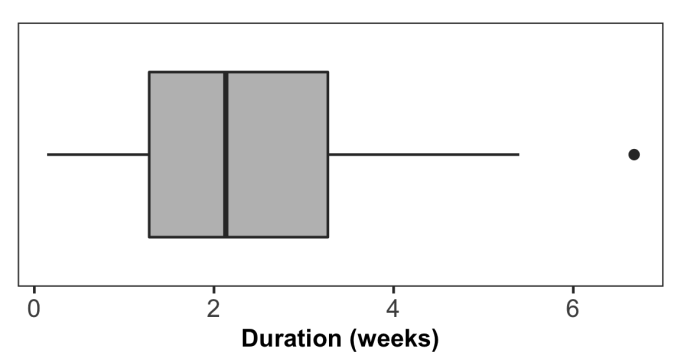

B

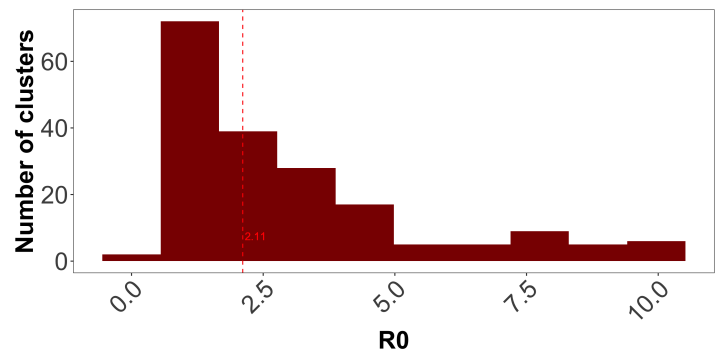

C

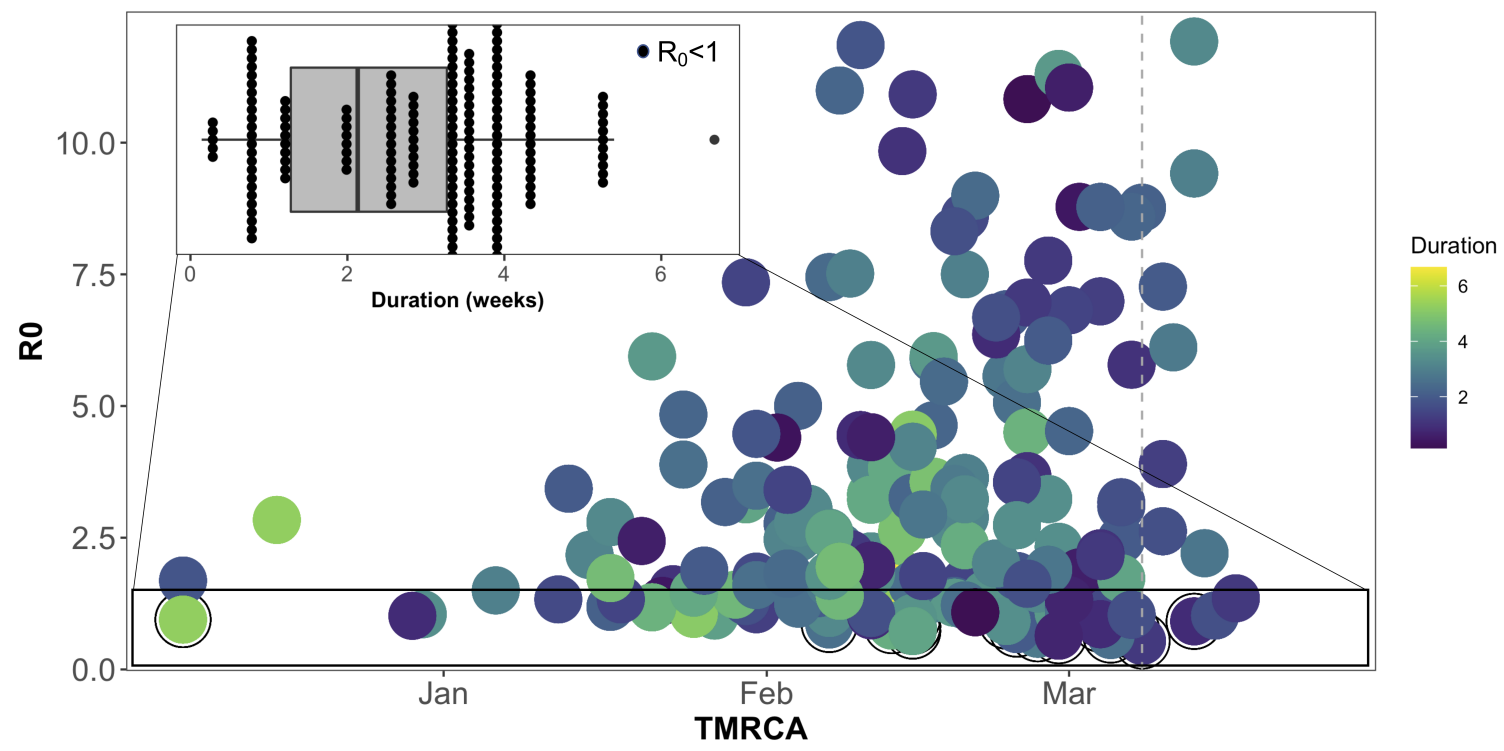

Figure 4: Estimated transmission cluster duration and $R_{0}$. (A) Duration was defined as the time from TMRCA to most recent sampling date for each cluster. (B) $R_{0}$ was derived from estimates of changes in effective population size, as described in (23). (C) Relationship of $R_{0}$ with time of cluster formation and cluster duration. Inset focuses on the distribution of $R_{0}<1$ clusters within the overall distribution of cluster duration. 
$\mathbf{A}$

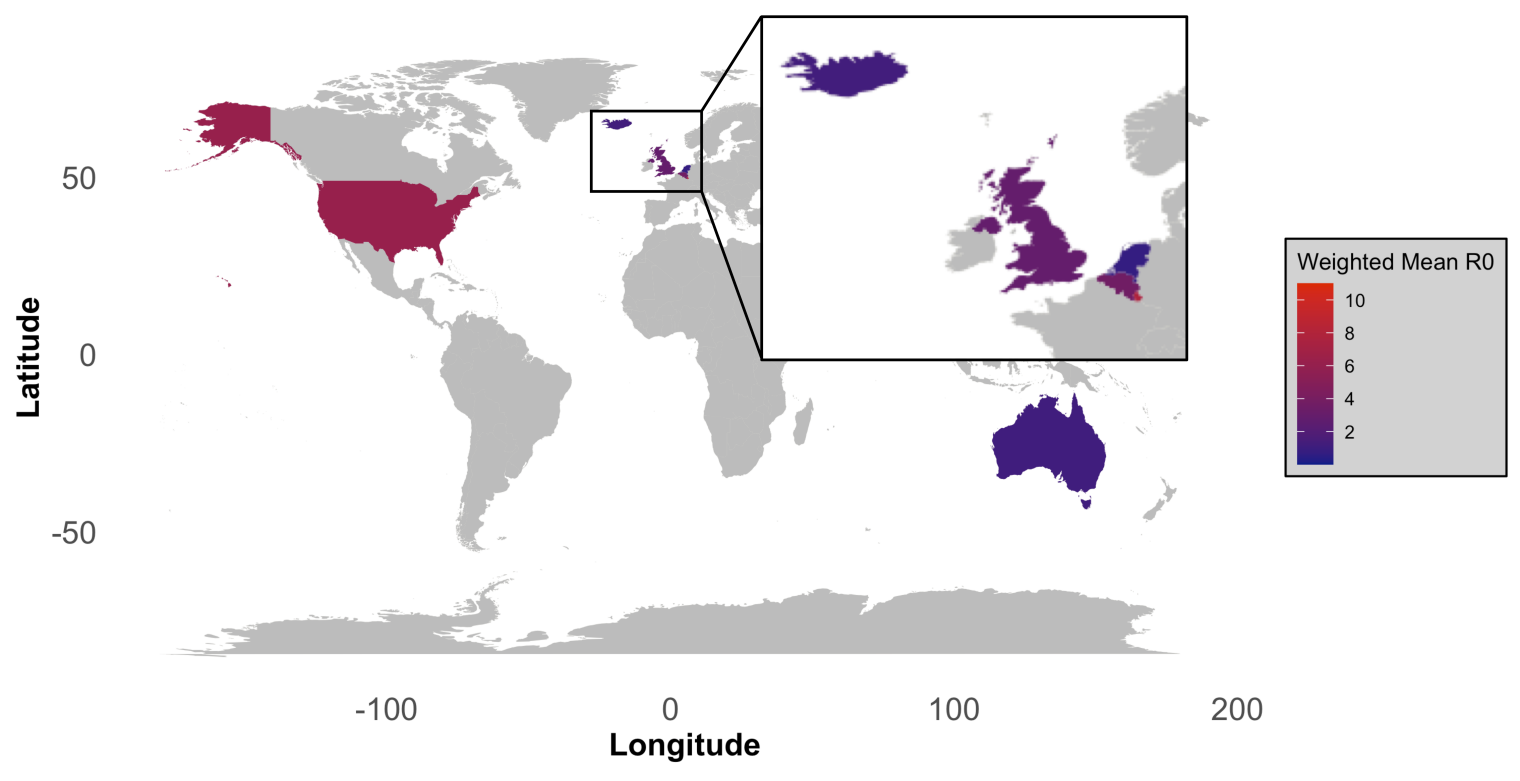

B

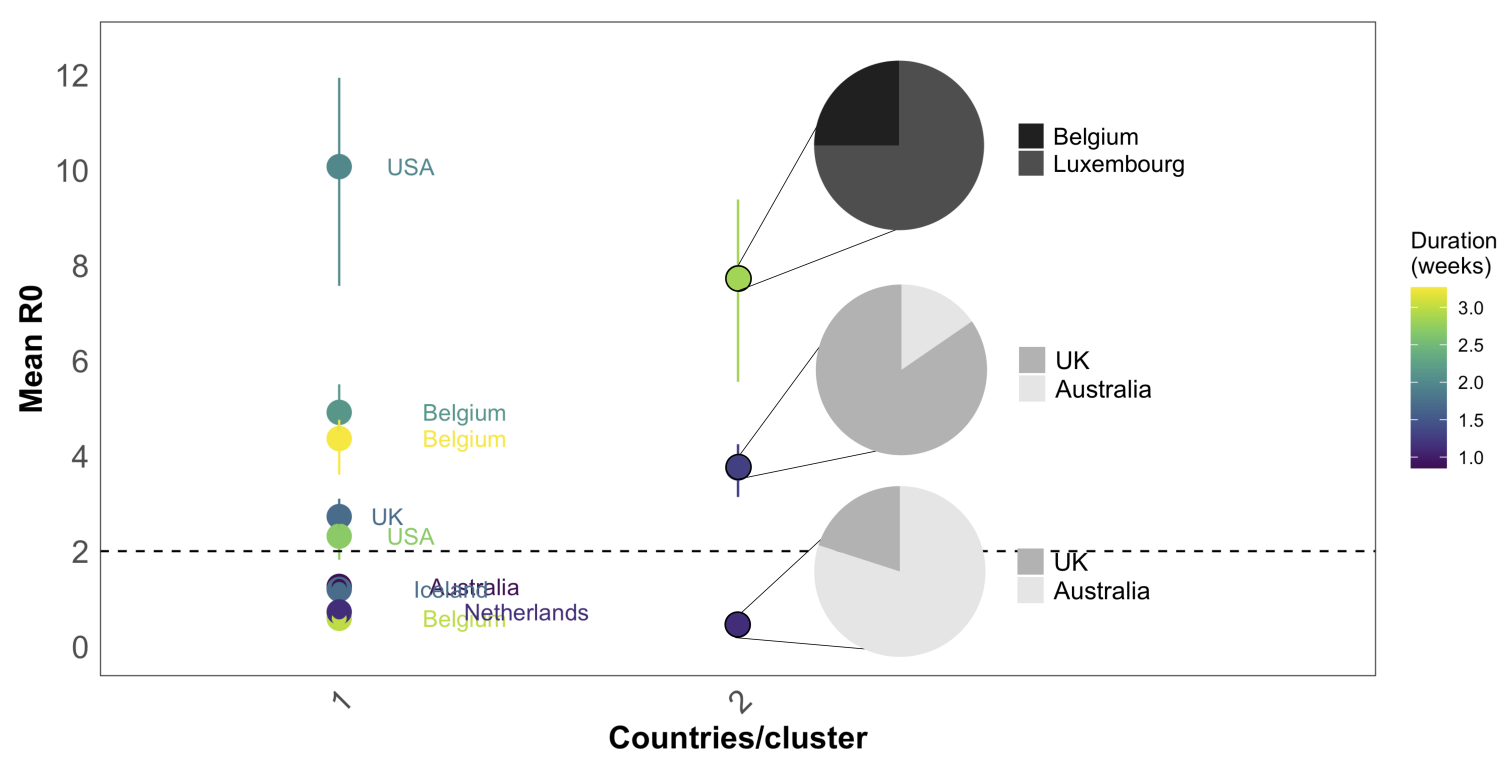

Figure 5: Summarized transmission patterns for countries involved in clusters formed after March 08, 2020. (A) Mean $R_{0}$ for each country was calculated by weighting each cluster-specific $R_{0}$ according to the fraction of sequences belonging to the country.(B) Estimated mean $R_{0}$ and $95 \%$ confidence intervals for each cluster (dot). For clusters with more than one country, the fraction of sequences belonging to each country is depicted in the corresponding pie chart. Clusters are also colored according to duration. Dashed line depicts $R_{0}=2$ 


\section{Supplementary Materials}

\section{Materials and Methods}

Sequence data, metadata, and phylogenetic reconstruction A total of 11,262 sequences and associated metadata (time and country of sampling) were downloaded from GISAID (GISAID) on April 25, 2020. Sequence IDs can be found in Table S3 The number of confirmed cases for each corresponding country was retrieved from European Centre for Disease Prevention and Control. Cruise ships Diamond Princess and Grand Princess were treated as separate geographical regions for all analyses. Data regarding travel restrictions were obtained from WorldAware, COVID-19 Travel Restrictions Database, and Trip.com on June 4th, 2020; data on border closures from The New York Times on June 1st, 2020; airline restrictions from Bloomberg and Business Insider on June 4th, 2020. Data specifically for the Diamond Princess was retrieved from Business Insider on June 5th, 2020. Data on nationwide lockdowns, health screening measures, closure of non-essential business, the use of masks and restrictions of mass gatherings were obtained from different online newspapers, including CNN, South China Morning Post, The New York Times, The Miami Herald, and Reuters on June 2nd.

Sequences were quality filtered and aligned, as described in (40), keeping one representative per identical sequence group and excluding sequences without precise dates (month and day), resulting in 11,316 sequences (29,726 nucleotides in length). We reconstructed a maximum likelihood tree with RAxML-NG (41) (GTR+I+G6 model, with all parameters optimized) starting from a distance tree (reconstructed using mimimum evolution in FASTME (42)), rooted it at the most recent common ancestor of the likely first-generation strains from (l), and put back the identical sequences (as zero-branch polytomies with the corresponding representative sequence tip). We then collapsed the branches without phylogenetic signal (i.e., of length $\leq 1 / 2$ mutation per genome). Support for branching events within the tree were calculated using the the 
Shimodaira-Hasegawa approximate likelihood ratio test (43), performed in IQ-TREE v2 (44) on the final, fixed RAxML-NG tree topology.

Least squares dating in LSD2 (45) was used to date internal nodes of the tree (given sampling dates of taxa) and to identify, and remove, outlier sequences. Outlier sequences were defined as taxa whose mutation rate (estimated as the distance to the root divided by time since the first sequence) was larger than 3 standard deviations from the median. A strict molecular clock was assumed, resulting in an estimated mutation rate of $2.3[2.0-2.4] \cdot 10^{4}$ mutations per site per year.

Transmission cluster identification Clades comprised of $\geq 5$ distinct sequences and a reliability of $\geq 90 \%$ were recognized as potential transmission clusters when the median pairwise patristic distance (i.e., branch length separating sequences) within the clade was below a pre-specified percentile threshold of the whole-tree patristic distance distribution. A range of percentile thresholds spanning $0.0005 \%-25 \%$ of the whole-tree distance distribution was used to choose an optimal threshold point and to verify robustness of cluster composition. The minimum percentile threshold that maximized the number of clusters was chosen as the optimal threshold by performing multiple clustering runs on randomly sampled patristic distance distributions (1 million for each run) in Phylopart v2 (46). Clusters and corresponding information (including sequence IDs) can be found in Table S4.

Potential clusters with more than two time points were used for the calculation of the basic reproductive number $\left(R_{0}\right)$ from the viral effective population size $\left(N_{e}\right)$ estimated using the skygrowth (23) package in R (47). Viral effective population size $\left(N_{e}\right)$ estimates were allowed to vary weekly, and the default smoothing parameter (tau) of 0.1 was used. As described in Volz Didelot (2018) (23), the mean effective reproductive number $\left(R_{e}\right)$ was calculated as a function of the change in $N e$ and 2-8 day (mean=5.475, SE=1.825) infectious period (psi). $R_{0}$ 
was defined as the first $R_{e}$ value in time. Clusters with outlying $R_{0}$ values $(>3.4$ standard deviations above or below the mean over all clusters) were discarded as unreliable.

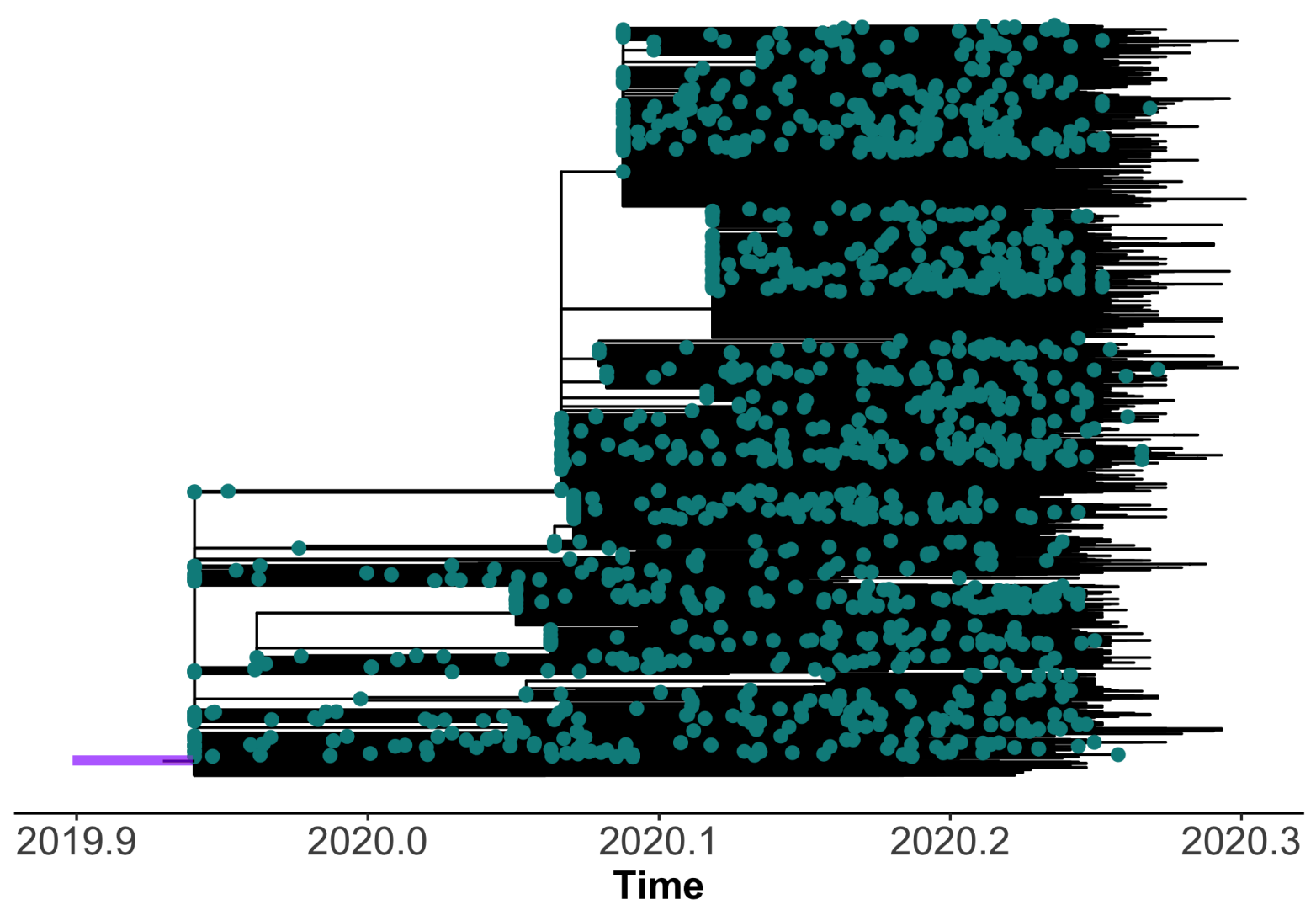

Figure S1: Maximum likelihood tree for 11,069 SARS-CoV-2 sequences scaled in time using least squares dating (45). Purple bar represents the $95 \%$ credible interval for estimate of the time to the most recent common ancestor for all sequences. Outlier sequences have been pruned and were excluded from downstream analyses (see Materials and Methods). 


\section{A}
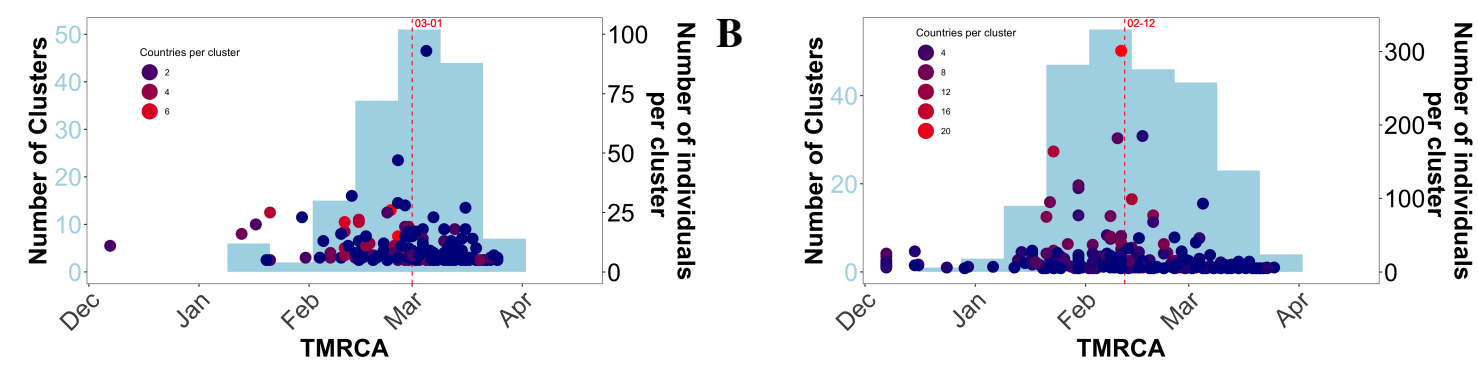

Figure S2: Timing of transmission cluster characteristics for additional distance thresholds defining transmission clusters. (A) Clusters with median patristic distances within $0.5 \%$ of the whole-tree patristic distance distribution. (B) Clusters with median patristic distances within $2.5 \%$ of the whole-tree patristic distance distribution. Bars represent the number of clusters (light blue) sharing a similar temporal origin, as inferred from the time to the most recent common ancestor (TMRCA) of corresponding cluster sequences. Dots correspond to the size (number of individuals) within the corresponding clusters at individual time points. Dots are colored according to the number of countries represented in each cluster. Red, dashed lines indicated median values.
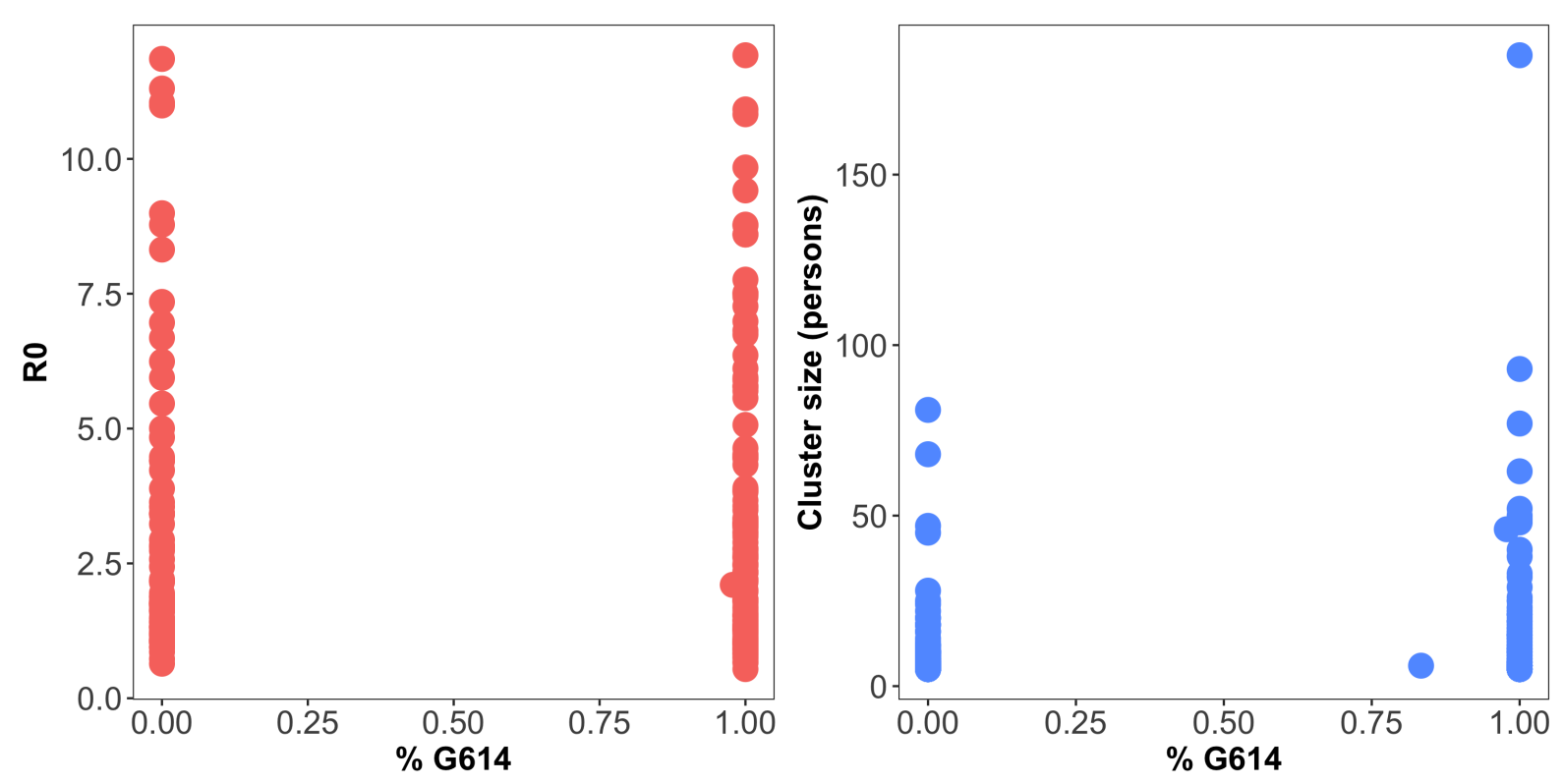

Figure S3: Relationship of $R_{0}(\mathrm{~A})$ and (B) cluster size with fraction of individuals with D614G mutation for each cluster. Each dot represents an individual cluster. Clusters in (A) represent a subset of clusters in (B) (see Materials and Methods). 
Table S1: Travel restriction data by country derived from various sources (see Materials and Methods)

\begin{tabular}{|c|c|c|c|c|c|c|c|c|c|c|}
\hline Country & Lockdown & $\begin{array}{l}\text { Travel ban } \\
\text { entering } \\
\text { from China }\end{array}$ & $\begin{array}{l}\text { Travel ban } \\
\text { entering } \\
\text { from Europe }\end{array}$ & $\begin{array}{l}\text { Travel ban } \\
\text { entering } \\
\text { from other } \\
\text { countries }\end{array}$ & $\begin{array}{c}\text { Non-citizens } \\
\text { banned/ } \\
\text { Travel } \\
\text { across } \\
\text { borders }\end{array}$ & $\begin{array}{c}\text { Health } \\
\text { screening } \\
\text { measures at } \\
\text { airports }\end{array}$ & $\begin{array}{l}\text { Education } \\
\text { closed or } \\
\text { moved } \\
\text { online }\end{array}$ & $\begin{array}{c}\text { Non } \\
\text { essential } \\
\text { business } \\
\text { closed }\end{array}$ & Masks & $\begin{array}{c}\text { Mass } \\
\text { gatherings }\end{array}$ \\
\hline Australia & $\begin{array}{c}\text { 3/23/2020 } \\
\text { nationwide }\end{array}$ & $2 / 2 / 20$ & $\begin{array}{l}3 / 12 / 2020 \\
\text { from Italy }\end{array}$ & $\begin{array}{c}3 / 1 / 2020 \\
\text { Iran and } \\
\text { South Korea }\end{array}$ & $3 / 25 / 20$ & $1 / 21 / 20$ & $3 / 16 / 20$ & $3 / 23 / 20$ & & $\begin{array}{c}3 / 20 / 2020 \\
\text { over } 100 \\
\text { people }\end{array}$ \\
\hline Belgium & $\begin{array}{c}\text { 3/17/2020 } \\
\text { nationwide }\end{array}$ & & & & $3 / 20 / 20$ & $1 / 30 / 20$ & $3 / 16 / 20$ & $3 / 13 / 20$ & $5 / 4 / 20$ & $\begin{array}{l}3 / 13 / 2020 \\
\text { all public } \\
\text { gatherings }\end{array}$ \\
\hline UK & $\begin{array}{c}\text { 3/23/2020 } \\
\text { nationwide }\end{array}$ & & & & $3 / 17 / 20$ & $1 / 30 / 20$ & $3 / 18 / 20$ & $3 / 21 / 20$ & & $3 / 17 / 20$ \\
\hline US & $\begin{array}{l}\text { 03/19/2020 } \\
\text { in California }\end{array}$ & $2 / 2 / 20$ & $\begin{array}{c}\text { 3/13/2020 } \\
\text { from } \\
\text { Schengen } \\
\text { area, } \\
\text { Austria, } \\
\text { Belgium } \\
\text { 3/17/2020 } \\
\text { from } \\
\text { Ireland, UK }\end{array}$ & $\begin{array}{c}2 / 29 / 2020 \\
\text { from Iran } \\
5 / 26 / 2020 \\
\text { from Brazil }\end{array}$ & $\begin{array}{c}3 / 21 / 2020 \\
\text { with Mexico } \\
\text { and Canada }\end{array}$ & $1 / 24 / 20$ & $\begin{array}{c}1 / 3 / 2020 \text { in } \\
\text { Florida }\end{array}$ & $\begin{array}{c}\text { 3/17/2020 in } \\
\text { California }\end{array}$ & $\begin{array}{c}\text { 4/17/2020 in } \\
\text { New York }\end{array}$ & $\begin{array}{l}3 / 11 / 2020 \text { in } \\
\text { Seattle over } \\
250 \text { people }\end{array}$ \\
\hline Brazil & $\begin{array}{c}\text { 3/17/2020 in } \\
\text { Santa } \\
\text { Catarina }\end{array}$ & $3 / 20 / 20$ & $\begin{array}{c}\text { 3/25/2020 } \\
\text { from } \\
\text { Norway, } \\
\text { Schengen } \\
\text { Area, } \\
\text { Switzerland, } \\
\text { UK, }\end{array}$ & $\begin{array}{l}\text { 3/25/2020 } \\
\text { from } \\
\text { Australia, } \\
\text { Iceland, } \\
\text { Japan, } \\
\text { Korea } \\
\text { (Rep.), } \\
\text { Malaysia, } \\
\text { Iran }\end{array}$ & $3 / 30 / 20$ & & $3 / 25 / 20$ & & $4 / 22 / 20$ & \\
\hline Canada & & & & & $3 / 16 / 20$ & $1 / 24 / 20$ & $3 / 23 / 20$ & $\begin{array}{c}3 / 17 / 2020 \text { in } \\
\text { Ottawa }\end{array}$ & $5 / 19 / 20$ & $\begin{array}{c}3 / 16 / 2020 \\
\text { over } 50 \\
\text { people } \\
\text { banned }\end{array}$ \\
\hline
\end{tabular}


Table S1: Travel restriction data by country derived from various sources (see Materials and Methods)

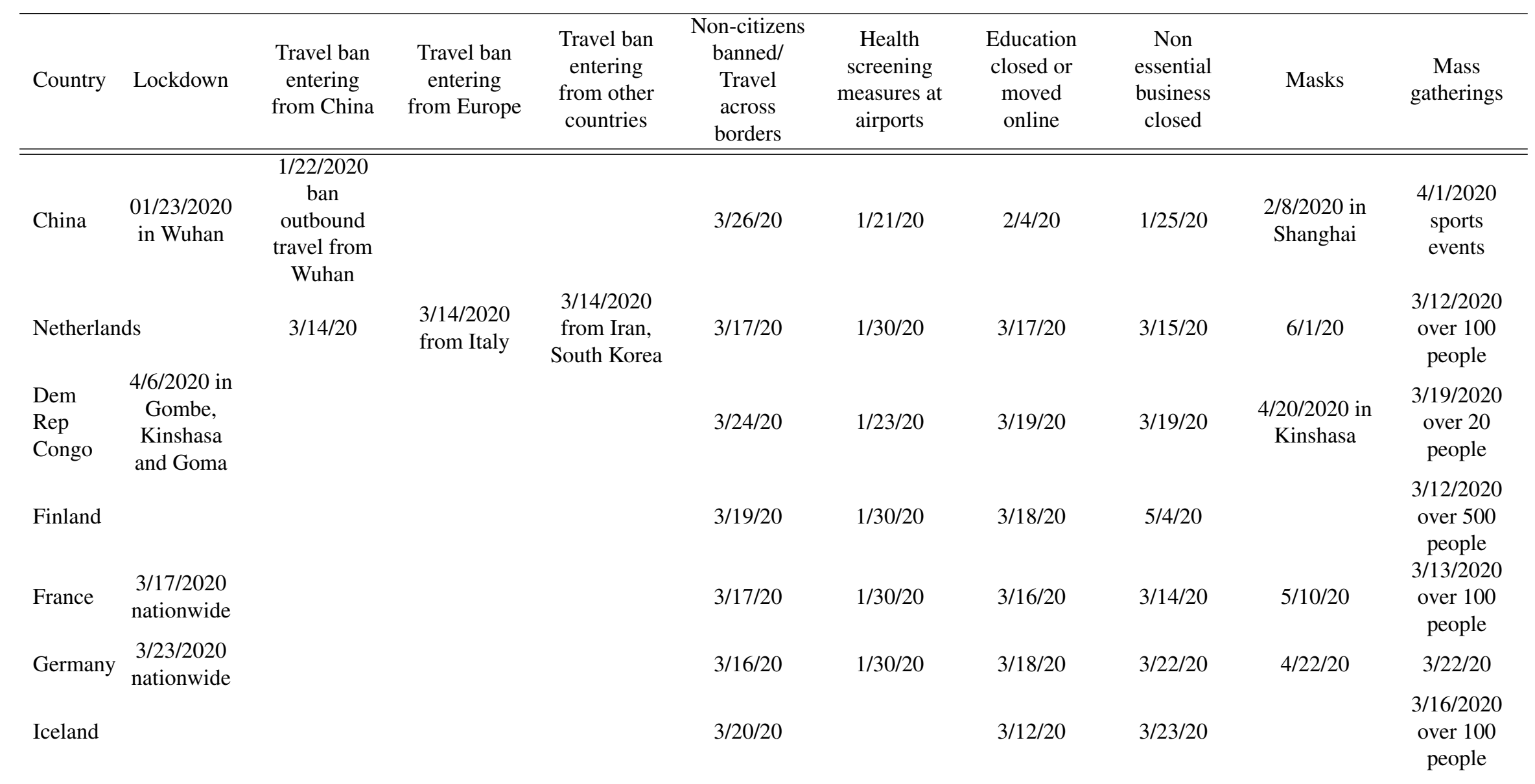


Table S1: Travel restriction data by country derived from various sources (see Materials and Methods)

\begin{tabular}{|c|c|c|c|c|c|c|c|c|c|c|}
\hline Country & Lockdown & $\begin{array}{l}\text { Travel ban } \\
\text { entering } \\
\text { from China }\end{array}$ & $\begin{array}{l}\text { Travel ban } \\
\text { entering } \\
\text { from Europe }\end{array}$ & $\begin{array}{l}\text { Travel ban } \\
\text { entering } \\
\text { from other } \\
\text { countries }\end{array}$ & $\begin{array}{c}\text { Non-citizens } \\
\text { banned/ } \\
\text { Travel } \\
\text { across } \\
\text { borders }\end{array}$ & $\begin{array}{l}\text { Health } \\
\text { screening } \\
\text { measures at } \\
\text { airports }\end{array}$ & $\begin{array}{c}\text { Education } \\
\text { closed or } \\
\text { moved } \\
\text { online }\end{array}$ & $\begin{array}{c}\text { Non } \\
\text { essential } \\
\text { business } \\
\text { closed }\end{array}$ & Masks & $\begin{array}{c}\text { Mass } \\
\text { gatherings }\end{array}$ \\
\hline
\end{tabular}


Table S1: Travel restriction data by country derived from various sources (see Materials and Methods)

\begin{tabular}{|c|c|c|c|c|c|c|c|c|c|c|}
\hline Country & Lockdown & $\begin{array}{l}\text { Travel ban } \\
\text { entering } \\
\text { from China }\end{array}$ & $\begin{array}{l}\text { Travel ban } \\
\text { entering } \\
\text { from Europe }\end{array}$ & $\begin{array}{l}\text { Travel ban } \\
\text { entering } \\
\text { from other } \\
\text { countries }\end{array}$ & $\begin{array}{c}\text { Non-citizens } \\
\text { banned/ } \\
\text { Travel } \\
\text { across } \\
\text { borders }\end{array}$ & $\begin{array}{c}\text { Health } \\
\text { screening } \\
\text { measures at } \\
\text { airports }\end{array}$ & $\begin{array}{l}\text { Education } \\
\text { closed or } \\
\text { moved } \\
\text { online }\end{array}$ & $\begin{array}{c}\text { Non } \\
\text { essential } \\
\text { business } \\
\text { closed }\end{array}$ & Masks & $\begin{array}{c}\text { Mass } \\
\text { gatherings }\end{array}$ \\
\hline Italy & $\begin{array}{c}3 / 8 / 2020 \\
\text { nationwide }\end{array}$ & $2 / 2 / 20$ & & & $3 / 28 / 20$ & $1 / 30 / 20$ & $3 / 10 / 20$ & $3 / 11 / 20$ & $5 / 4 / 20$ & $3 / 8 / 20$ \\
\hline
\end{tabular}


Table S1: Travel restriction data by country derived from various sources (see Materials and Methods)

\begin{tabular}{|c|c|c|c|c|c|c|c|c|c|c|}
\hline Country & Lockdown & $\begin{array}{l}\text { Travel ban } \\
\text { entering } \\
\text { from China }\end{array}$ & $\begin{array}{l}\text { Travel ban } \\
\text { entering } \\
\text { from Europe }\end{array}$ & $\begin{array}{l}\text { Travel ban } \\
\text { entering } \\
\text { from other } \\
\text { countries }\end{array}$ & $\begin{array}{c}\text { Non-citizens } \\
\text { banned/ } \\
\text { Travel } \\
\text { across } \\
\text { borders }\end{array}$ & $\begin{array}{c}\text { Health } \\
\text { screening } \\
\text { measures at } \\
\text { airports }\end{array}$ & $\begin{array}{l}\text { Education } \\
\text { closed or } \\
\text { moved } \\
\text { online }\end{array}$ & $\begin{array}{c}\text { Non } \\
\text { essential } \\
\text { business } \\
\text { closed }\end{array}$ & Masks & $\begin{array}{l}\text { Mass } \\
\text { gatherings }\end{array}$ \\
\hline
\end{tabular}

$3 / 26 / 2020$
from Iran

Andorra,

Austria,

Belgium,

Denmark,

Estonia,

France,

Germany,

Liechten-

stein,

Luxem-

bourg,

Malta,

Monaco,

Netherlands,

Norway,

Poland, San

Marino,

Slovenia,

Sweden,

Vatican City

State

4/2/2020

from

Albania,

Armenia,

Australia,

Bolivia,

Bosnia and

Herzegov-

ina, Brazil,

Canada,

Chile, Côte

d'Ivoire

(Ivory

Coast), 
Table S1: Travel restriction data by country derived from various sources (see Materials and Methods)

\begin{tabular}{|c|c|c|c|c|c|c|c|c|c|c|}
\hline Country & Lockdown & $\begin{array}{l}\text { Travel ban } \\
\text { entering } \\
\text { from China }\end{array}$ & $\begin{array}{l}\text { Travel ban } \\
\text { entering } \\
\text { from Europe }\end{array}$ & $\begin{array}{l}\text { Travel ban } \\
\text { entering } \\
\text { from other } \\
\text { countries }\end{array}$ & $\begin{array}{c}\text { Non-citizens } \\
\text { banned/ } \\
\text { Travel } \\
\text { across } \\
\text { borders }\end{array}$ & $\begin{array}{c}\text { Health } \\
\text { screening } \\
\text { measures at } \\
\text { airports }\end{array}$ & $\begin{array}{l}\text { Education } \\
\text { closed or } \\
\text { moved } \\
\text { online }\end{array}$ & $\begin{array}{c}\text { Non } \\
\text { essential } \\
\text { business } \\
\text { closed }\end{array}$ & Masks & $\begin{array}{c}\text { Mass } \\
\text { gatherings }\end{array}$ \\
\hline \multicolumn{2}{|c|}{ Luxembourg } & & & & $\begin{array}{c}3 / 16 / 2020 \\
\text { with } \\
\text { Germany }\end{array}$ & $1 / 24 / 20$ & $3 / 16 / 20$ & $3 / 16 / 20$ & $4 / 20 / 20$ & \multirow[t]{2}{*}{$\begin{array}{c}3 / 13 / 2020 \\
\text { over } 100 \\
\text { people }\end{array}$} \\
\hline Mexico & & & & & $\begin{array}{l}3 / 21 / 2020 \\
\text { with USA }\end{array}$ & $1 / 24 / 20$ & $3 / 23 / 20$ & $3 / 30 / 20$ & & \\
\hline Portugal & $\begin{array}{c}\text { 3/19/2020 } \\
\text { nationwide }\end{array}$ & & $\begin{array}{c}3 / 11 / 2020 \\
\text { from } \\
\text { Italy3/18/2020 } \\
\text { from Spain }\end{array}$ & & & & $3 / 16 / 20$ & $3 / 13 / 20$ & $5 / 4 / 20$ & $\begin{array}{c}3 / 15 / 2020 \\
\text { over } 100 \\
\text { people }\end{array}$ \\
\hline Russia & $\begin{array}{l}3 / 28 / 2020 \\
\text { nationwide }\end{array}$ & $1 / 28 / 20$ & $\begin{array}{l}\text { 3/13/2020 } \\
\text { Italy }\end{array}$ & $\begin{array}{l}\text { 3/4/2020 } \\
\text { from Iran, } \\
\text { South Korea }\end{array}$ & $\begin{array}{c}3 / 14 / 2020 \\
\text { with Poland } \\
\text { and Norway }\end{array}$ & $1 / 30 / 20$ & $3 / 16 / 20$ & $3 / 28 / 20$ & $5 / 11 / 20$ & $\begin{array}{c}3 / 10 / 2020 \\
\text { over } 5000 \\
\text { people in } \\
\text { Moscow }\end{array}$ \\
\hline Singapore & $\begin{array}{c}\text { 4/7/2020 } \\
\text { nationwide }\end{array}$ & $\begin{array}{c}\text { 1/31/2020 } \\
\text { 2/3/2020 } \\
\text { from Hong } \\
\text { Kong, } \\
\text { Macau }\end{array}$ & $\begin{array}{c}3 / 4 / 2020 \\
\text { from Italy, } \\
\text { Iran, Korea } \\
\text { (Rep) } \\
3 / 15 / 2020 \\
\text { from } \\
\text { France, } \\
\text { Germany, } \\
\text { and Spain }\end{array}$ & & & & & $4 / 7 / 20$ & $4 / 14 / 20$ & $\begin{array}{c}3 / 24 / 2020 \\
\text { over } 10 \\
\text { people }\end{array}$ \\
\hline Spain & $\begin{array}{l}\text { 3/14/2020 } \\
\text { nationwide }\end{array}$ & & $\begin{array}{l}3 / 13 / 2020 \\
\text { from Italy }\end{array}$ & & $3 / 17 / 20$ & $1 / 30 / 20$ & $3 / 16 / 20$ & $3 / 14 / 20$ & $5 / 4 / 20$ & $\begin{array}{c}2 / 12 / 2020 \\
\text { over } 1000 \\
\text { people in } \\
\text { Madrid }\end{array}$ \\
\hline Sweden & & & & & $3 / 19 / 20$ & $1 / 30 / 20$ & $\begin{array}{c}3 / 18 / 2020 \\
\text { some locally }\end{array}$ & & & $\begin{array}{c}3 / 27 / 2020 \\
\text { over } 50 \\
\text { people }\end{array}$ \\
\hline
\end{tabular}


Table S1: Travel restriction data by country derived from various sources (see Materials and Methods)

\begin{tabular}{|c|c|c|c|c|c|c|c|c|c|c|}
\hline Country & Lockdown & $\begin{array}{l}\text { Travel ban } \\
\text { entering } \\
\text { from China }\end{array}$ & $\begin{array}{l}\text { Travel ban } \\
\text { entering } \\
\text { from Europe }\end{array}$ & $\begin{array}{l}\text { Travel ban } \\
\text { entering } \\
\text { from other } \\
\text { countries }\end{array}$ & $\begin{array}{c}\text { Non-citizens } \\
\text { banned/ } \\
\text { Travel } \\
\text { across } \\
\text { borders }\end{array}$ & $\begin{array}{c}\text { Health } \\
\text { screening } \\
\text { measures at } \\
\text { airports }\end{array}$ & $\begin{array}{l}\text { Education } \\
\text { closed or } \\
\text { moved } \\
\text { online }\end{array}$ & $\begin{array}{l}\text { Non } \\
\text { essential } \\
\text { business } \\
\text { closed }\end{array}$ & Masks & $\begin{array}{c}\text { Mass } \\
\text { gatherings }\end{array}$ \\
\hline Switzerlan & & $2 / 28 / 20$ & $\begin{array}{l}\text { 3/17/2020 } \\
\text { with Italy, } \\
\text { Austria, } \\
\text { France, } \\
\text { Germany }\end{array}$ & & $3 / 26 / 20$ & & $3 / 16 / 20$ & $3 / 16 / 20$ & & $\begin{array}{c}3 / 2 / 2020 \\
\text { over } 1000 \\
\text { people }\end{array}$ \\
\hline Taiwan & & $2 / 4 / 20$ & & & $3 / 19 / 20$ & $1 / 22 / 20$ & $2 / 21 / 20$ & & $4 / 1 / 20$ & $\begin{array}{c}\text { 2/29/2020 in } \\
\text { Taipei }\end{array}$ \\
\hline $\begin{array}{l}\text { Diamond } \\
\text { Princess } \\
\text { Grand } \\
\text { Princess }\end{array}$ & $\begin{array}{l}2 / 1 / 2020 \\
\text { quarantined, } \\
\text { docked in } \\
\text { Okinawa } \\
3 / 4 / 2020 \\
\text { CDC orders } \\
\text { some } \\
\text { passengers } \\
\text { self- } \\
\text { quarantine }\end{array}$ & & & & & & & & & \\
\hline
\end{tabular}


Table S2: Airline travel data obtained from various sources (see Materials and Methods).

\begin{tabular}{|c|c|c|c|}
\hline Airline & Base & Date & Ban \\
\hline Cabo Verde Airlines & Africa & $3 / 18 / 20$ & Suspending all flights \\
\hline SA express & Africa & $3 / 18 / 20$ & Suspending all flights \\
\hline Aerolineas Argentina & Argentina & $3 / 18 / 20$ & Cut capacity to Rome, Madrid, Miami, New York and Orlando (various dates) \\
\hline Qantas Airways & Australia & 2/9/20 & $\begin{array}{l}\text { Flights to Beijing and Shanghai; cut capacity to Hong Kong, Sapporo, Auck- } \\
\text { land, Tokyo and Osaka }\end{array}$ \\
\hline Tigerair Australia & Australia & $3 / 31 / 20$ & Suspending all flights \\
\hline Virgins Australia & Australia & $2 / 11 / 20$ & $\begin{array}{l}\text { Permanent cancellation of routes to Hong Kong from Melbourne (from Feb. } \\
\text { 11) and Sydney }\end{array}$ \\
\hline Austrian Airlines & Austria & $1 / 29 / 20$ & $\begin{array}{l}\text { Flights to Venice, Bologna, Milan, Beijing, Shanghai, Tel Aviv and Iran; cut } \\
\text { capacity to European routes by } 20 \%\end{array}$ \\
\hline Bahamas Air & Bahamas & $3 / 23 / 20$ & Suspending all flights \\
\hline Gulf Air & Bahrain & $2 / 27 / 20$ & Flights to Dubai, Iraq and Lebanon; cut capacity to several other destinations \\
\hline Air Antwerp & Belgium & $3 / 22 / 20$ & Canceling flights until April 22 \\
\hline Brussels Airline & Belgium & $3 / 12 / 20$ & All flights to and from Italy \\
\hline LATAM Airlines Brasil & Brazil & $3 / 2 / 20$ & Flights between Sao Paulo and Milan \\
\hline Air Canada & Canada & $1 / 30 / 20$ & $\begin{array}{l}\text { All flights to and from mainland China and Italy; flights between Toronto and } \\
\text { Hong Kong; maintaining flights between Vancouver and Hong Kong }\end{array}$ \\
\hline Air Transat & Canada & $3 / 13 / 20$ & Gradually suspending all flights \\
\hline Harbour Air & Canada & $3 / 27 / 20$ & Suspending all flights \\
\hline Porter Airlines & Canada & $3 / 21 / 20$ & Suspending all flights \\
\hline Sunwing Airlines & Canada & $3 / 17 / 20$ & Suspending all flights \\
\hline Winair & Caribbean & $3 / 19 / 20$ & Suspending all flights \\
\hline Avianca & Colombia & $3 / 25 / 20$ & Suspending all flights \\
\hline CSA Czech Airlines & Czech & $3 / 16 / 20$ & Suspending all flights \\
\hline TAME & Ecuador & $3 / 17 / 20$ & Suspending all flights \\
\hline EgyptAir & Egypt & $3 / 31 / 20$ & Suspending all flights \\
\hline Ethiopian Airlines & Ethiopia & $2 / 3 / 20$ & $\begin{array}{l}\text { Reduced capacity and total number of flights to Beijing, Shanghai, } \\
\text { Guangzhou and Hong Kong }\end{array}$ \\
\hline
\end{tabular}


Table S2: Airline travel data obtained from various sources (see Materials and Methods).

\begin{tabular}{|c|c|c|c|}
\hline Airline & Base & Date & Ban \\
\hline Finnair & Finland & $3 / 6 / 20$ & $\begin{array}{l}\text { All flights to mainland China; cut capacity to Guangzhou, Hong Kong, Osaka, } \\
\text { Seoul, Milan; cut capacity across European network by } 20 \% \text {; new route to } \\
\text { Busan postponed }\end{array}$ \\
\hline Air France & France & $1 / 30 / 20$ & $\begin{array}{l}\text { All scheduled flights to mainland China, Hong Kong, Taipei, Italy, Tel Aviv; } \\
\text { will gradually resume operations to Beijing, Shanghai, Hong Kong and Taipei } \\
\text { after March 29; cut capacity to Seoul, Singapore, Japan, and across Europe }\end{array}$ \\
\hline La Champagnie & France & $3 / 18 / 20$ & Suspending all flights \\
\hline Transavia & France & $3 / 23 / 20$ & Suspending all flights \\
\hline Lufthansa & Germany & $1 / 30 / 20$ & $\begin{array}{l}\text { Flights to Beijing, Shanghai, Nanjing, Shenyang, Qingdao, Tehran and Israel; } \\
\text { cut capacity to Hong Kong, Seoul, Italy, various locations throughout Europe } \\
\text { and domestically; cut flight capacity by up to } 50 \%\end{array}$ \\
\hline Cathay Pacific & $\begin{array}{l}\text { Hong } \\
\text { Kong }\end{array}$ & $1 / 30 / 20$ & $\begin{array}{l}\text { Flights to Japan, Tel Aviv, Jeju, Busan, Kaohsiung, Taichung, Seoul, Wash- } \\
\text { ington, London Gatwick, Rome; cut capacity of flights to and from mainland } \\
\text { China by } 90 \% \text {; cut global capacity by about } 40 \%\end{array}$ \\
\hline Wizz Air & Hungary & $3 / 11 / 20$ & $\begin{array}{l}\text { All flights to Italy; flights between Luton and Tel Aviv; cut capacity to various } \\
\text { destinations }\end{array}$ \\
\hline Air India & India & $1 / 31 / 20$ & Flights between Delhi and Shanghai, and between Delhi and Hong Kong \\
\hline IndiGO & India & $2 / 1 / 20$ & $\begin{array}{l}\text { Flights between Bengaluru and Hong Kong, between Delhi and Chengdu, and } \\
\text { between Kolkata and Guangzhou (various suspension dates) }\end{array}$ \\
\hline Spice Jet & India & $3 / 25 / 20$ & Suspending all flights \\
\hline Vistara & India & $3 / 25 / 20$ & Suspending all flights \\
\hline Iran Air & Iran & $3 / 8 / 20$ & $\begin{array}{l}\text { Flights to Vienna, Stockholm and Gothenburg; temporarily suspended all } \\
\text { flights to Europe }\end{array}$ \\
\hline Aer Lingus & Ireland & $3 / 11 / 20$ & All flights to and from Italy \\
\hline Lauda & Ireland & $3 / 16 / 20$ & Gradually suspending all flights \\
\hline Ryanair & Ireland & $3 / 17 / 20$ & All flights to and from Italy \\
\hline El Al Israel & Israel & $3 / 27 / 20$ & Suspending all flights \\
\hline Neos & Italy & $1 / 30 / 20$ & All flights to and from China \\
\hline
\end{tabular}


Table S2: Airline travel data obtained from various sources (see Materials and Methods).

\begin{tabular}{|c|c|c|c|}
\hline Airline & Base & Date & Ban \\
\hline All Nippon Airways & Japan & $1 / 23 / 20$ & $\begin{array}{l}\text { Flights to nine cities in China, including Beijing, Shanghai and Guangzhou, } \\
\text { from Tokyo and Osaka; flights between Osaka and Hong Kong; cut capacity } \\
\text { to four cities in China and various domestic routes }\end{array}$ \\
\hline Japan Airlines & Japan & $2 / 6 / 20$ & $\begin{array}{l}\text { Flights from Haneda to Guangzhou, Narita to Hong Kong Kong and Hon- } \\
\text { olulu, Tokyo to Beijing, Shanghai and Dalian; cut capacity to some South } \\
\text { Korea, Taiwan and domestic routes }\end{array}$ \\
\hline $\begin{array}{l}\text { Royal Jordanian Air- } \\
\text { lines }\end{array}$ & Jordan & $3 / 17 / 20$ & Suspending all flights \\
\hline Air Astana & Kazakhstan & $4 / 15 / 20$ & Restrict flights in the country \\
\hline Qazaq Airlines & Kazakhstan & $4 / 15 / 20$ & Suspending all flights \\
\hline Kenya Airlines & Kenya & $1 / 31 / 20$ & Flights to and from Guangzhou \\
\hline Kuwait & Kuwait & $3 / 6 / 20$ & Suspending all flights \\
\hline Air Baltic & Latvia & $3 / 17 / 20$ & Suspending all flights Latvia, Lithuania, and Estonia \\
\hline Middle East Airlines & Lebanon & $3 / 19 / 20$ & Suspending all flights \\
\hline Luxair & Luxembourg & $3 / 24 / 20$ & Suspending all flights \\
\hline Air Madagascar & Madagascar & $3 / 20 / 20$ & Suspending all flights \\
\hline Air Asia & Malaysia & $1 / 24 / 20$ & $\begin{array}{l}\text { All flights to Wuhan and selected flights to mainland China; all flights be- } \\
\text { tween the Philippines and mainland China, Hong Kong and Macau }\end{array}$ \\
\hline Air Malta & Malta & $4 / 2 / 20$ & Suspending all flights \\
\hline Air Moldova & Moldova & $4 / 1 / 20$ & Suspending all flights \\
\hline FlyOne & Moldova & $3 / 17 / 20$ & Suspending all flights \\
\hline Montenegro Airlines & Montenegro & $3 / 16 / 20$ & Suspending all flights \\
\hline Royal Air Maroc & Morocco & $1 / 31 / 20$ & Direct flights between Casablanca and Beijing \\
\hline KLM & Netherlands & $1 / 30 / 20$ & $\begin{array}{l}\text { All flights to Italy; flights to Chengdu, Hangzhou and Xiamen and to Beijing } \\
\text { and Shanghai; cut capacity to Hong Kong }\end{array}$ \\
\hline Air New Zealand & $\begin{array}{l}\text { New } \\
\text { Zealand }\end{array}$ & $2 / 9 / 20$ & $\begin{array}{l}\text { Flights between Auckland and Shanghai; cut capacity to Shanghai and Hong } \\
\text { Kong }\end{array}$ \\
\hline
\end{tabular}


Table S2: Airline travel data obtained from various sources (see Materials and Methods).

\begin{tabular}{|c|c|c|c|}
\hline Airline & Base & Date & Ban \\
\hline Norwegian Air Shuttles & Norway & $3 / 15 / 20$ & $\begin{array}{l}\text { All flights to U.S. from Rome, Paris, Barcelona, Madrid, Amsterdam, Athens, } \\
\text { Oslo; all flights to Italy; majority of flights to U.S. from Amsterdam and } \\
\text { Stockholm; large share of flights within Scandinavia; maintaining London to } \\
\text { U.S. route }\end{array}$ \\
\hline Copa Airlines & Panama & $3 / 23 / 20$ & Suspending all flights \\
\hline Cebu Airline & Philippines & $2 / 2 / 20$ & Flights to mainland China and Taiwan, cut capacity to Hong Kong and Macau \\
\hline Philippine Airlines & Philippines & $3 / 26 / 20$ & Suspending all flights \\
\hline TAP Air Portugal & Portugal & $3 / 5 / 20$ & Cut 2,500 flights, mostly to European destinations like Italy, Spain and France \\
\hline Qatar Airways & Qatar & $2 / 3 / 20$ & All flights to mainland China \\
\hline Blue Air & Romania & $3 / 21 / 20$ & Suspending all flights \\
\hline Rwandair & Rwanda & $1 / 31 / 20$ & Flights between Kigali and Guangzhou \\
\hline Saudia & $\begin{array}{l}\text { Saudi } \\
\text { Arabia }\end{array}$ & $2 / 2 / 20$ & All flights to China \\
\hline Air Seychelles & Seychelles & $3 / 9 / 20$ & Flights to Tel Aviv, Mumbai, Johannesburg and Mauritius \\
\hline Jetstar Airways & Singapore & $3 / 23 / 20$ & Suspending all flights \\
\hline Scoot & Singapore & $2 / 23 / 20$ & $\begin{array}{l}\text { Flights to } 19 \text { cities to China including Wuhan, Guangzhou and Xi' an; ad-hoc } \\
\text { cancellations of flights to Hong Kong and Macau }\end{array}$ \\
\hline SilkAir & Singapore & $2 / 4 / 20$ & Flights to Shenzhen, Xiamen, Chongqing and Hiroshima \\
\hline Singapore Airlines & Singapore & $2 / 3 / 20$ & $\begin{array}{l}\text { Flights to Beijing, Shanghai and Guangzhou; cut capacity to Hong Kong, Los } \\
\text { Angeles, London, Paris and dozens of other cities }\end{array}$ \\
\hline Airlink & $\begin{array}{l}\text { South } \\
\text { Africa }\end{array}$ & $3 / 28 / 20$ & Suspending all flights \\
\hline
\end{tabular}


Table S2: Airline travel data obtained from various sources (see Materials and Methods).

\begin{tabular}{|c|c|c|c|}
\hline Airline & Base & Date & Ban \\
\hline Kulula & $\begin{array}{l}\text { South } \\
\text { Africa }\end{array}$ & $3 / 26 / 20$ & Suspending all flights \\
\hline Air Seoul & $\begin{array}{l}\text { South } \\
\text { Korea }\end{array}$ & $1 / 28 / 20$ & $\begin{array}{l}\text { Flights between Incheon and Zhangjiajie and Linyi in China; between In- } \\
\text { cheon and Hanoi }\end{array}$ \\
\hline Asiana Airlines & $\begin{array}{l}\text { South } \\
\text { Korea }\end{array}$ & $2 / 4 / 20$ & $\begin{array}{l}\text { Flights to dozens of Asian cities including Beijing, Guangzhou and Hong } \\
\text { Kong; between Daegu and Jeju; flights to Venice, Rome and Barcelona; cut } \\
\text { capacity to } 15 \text { cities, including Shanghai and Shenzhen (various suspension } \\
\text { dates) }\end{array}$ \\
\hline Korean Air & $\begin{array}{l}\text { South } \\
\text { Korea }\end{array}$ & $1 / 24 / 20$ & $\begin{array}{l}\text { Flights between Incheon and Wuhan, Huangshan, Zhangjiajie, Changsha, } \\
\text { Kunming, Tel Aviv, Daegu; to Hanoi, Danang, Phu Quoc, Bangkok, Siem } \\
\text { Reap, Taipei, Chubu, Aomori and Ulaanbaatar; between Busan and Bei- } \\
\text { jing/Nanjing; between Jeju and Daegu. Also reduced frequency to } 15 \text { more } \\
\text { routes }\end{array}$ \\
\hline T’way Air & $\begin{array}{l}\text { South } \\
\text { Korea }\end{array}$ & $2 / 5 / 20$ & Flights between South Korea and Hanoi \\
\hline Iberia & Spain & $1 / 31 / 20$ & Flights between Madrid and Shanghai \\
\hline $\begin{array}{l}\text { Swiss International Air- } \\
\text { lines }\end{array}$ & Switzerland & $1 / 29 / 20$ & Flights to Beijing and Shanghai \\
\hline China Airlines & Taiwan & $1 / 23 / 20$ & Flights to and from Wuhan \\
\hline Starlux & Taiwan & $3 / 21 / 20$ & Suspending all flights \\
\hline Thai Lion Air & Thailand & $3 / 25 / 20$ & Suspending all flights \\
\hline Pegasus Airlines & Turkey & $2 / 25 / 20$ & Flights between Istanbul and Tehran \\
\hline Sunexpress & Turkey & $3 / 27 / 20$ & Suspending all flights \\
\hline Turkish Airlines & Turkey & $1 / 31 / 20$ & $\begin{array}{l}\text { Flights to mainland China and Iran, except Tehran, Nakhcevan, Azerbaijan; } \\
\text { cut capacity to Israel, Seoul and Nigeria }\end{array}$ \\
\hline Turkmenistan Airlines & Turkmenistan & $2 / 1 / 20$ & Flights to Beijing \\
\hline Emirates & UAE & $3 / 25 / 20$ & Suspending all flights \\
\hline
\end{tabular}


Table S2: Airline travel data obtained from various sources (see Materials and Methods).

\begin{tabular}{|c|c|c|c|}
\hline Airline & Base & Date & Ban \\
\hline Etihad Airways & UAE & $2 / 5 / 20$ & $\begin{array}{l}\text { Flights to Rome, Milan, Shanghai and Chengdu; maintaining service to Bei- } \\
\text { jing }\end{array}$ \\
\hline Fly Dubai & UAE & $3 / 25 / 20$ & Suspending all flights \\
\hline British Airways & UK & $1 / 29 / 20$ & $\begin{array}{l}\text { All flights to Italy, Beijing and Shanghai; cut capacity to New York, Singa- } \\
\text { pore, France, Austria, Belgium, Germany, Ireland, Switzerland and Albania }\end{array}$ \\
\hline Cayman Airline & UK & $3 / 22 / 20$ & Suspending all flights \\
\hline Comair British Airlines & UK & $3 / 26 / 20$ & Suspending all flights \\
\hline easyJet & UK & $3 / 24 / 20$ & Suspending all flights \\
\hline Jet2Go.com & UK & $3 / 17 / 20$ & Suspending all flights \\
\hline Virgina Atlantic & UK & $2 / 1 / 20$ & $\begin{array}{l}\text { Flights to Shanghai; maintaining Hong Kong service; new Sao Paulo route } \\
\text { postponed }\end{array}$ \\
\hline $\begin{array}{l}\text { Ukraines International } \\
\text { Airlines }\end{array}$ & Ukraine & $3 / 17 / 20$ & Suspending all flights \\
\hline Air Arabia & $\begin{array}{l}\text { United } \\
\text { Arab } \\
\text { Emirates }\end{array}$ & $3 / 25 / 20$ & Banned passengers from entering \\
\hline American Airlines & USA & $1 / 31 / 20$ & $\begin{array}{l}\text { All flights to China and Argentina; Hong Kong service to Dallas and Los } \\
\text { Angeles; Seoul service to Dallas; Milan service to New York and Miami; } \\
\text { Rome service to Philadelphia, Chicago and Charlotte; Santiago service to } \\
\text { Dallas; cut capacity to Paris, Madrid, Montevideo, Barcelona, Venice; cut } \\
\text { summer international capacity by half }\end{array}$ \\
\hline Delta & USA & $2 / 2 / 20$ & $\begin{array}{l}\text { All flights to China and Italy; cut capacity to South Korea, Japan, EU coun- } \\
\text { tries and Latin America }\end{array}$ \\
\hline United Airlines & USA & $2 / 5 / 20$ & $\begin{array}{l}\text { Flights to Beijing, Shanghai, Chengdu and Hong Kong; also flights from Los } \\
\text { Angeles, Houston and Chicago to Tokyo Narita; reduced capacity and sched- } \\
\text { ule for flights from Newark, Honolulu and San Francisco to Tokyo Narita, } \\
\text { Osaka Kansai, Singapore, Seoul and Taipei }\end{array}$ \\
\hline Uzbekistan Airways & Uzbekistan & $3 / 17 / 20$ & Suspending all flights \\
\hline
\end{tabular}


Table S2: Airline travel data obtained from various sources (see Materials and Methods).

\begin{tabular}{lccl}
\hline Airline & Base & Date & Ban \\
\hline \hline VietJet Air & Vietnam & $2 / 1 / 20$ & $\begin{array}{l}\text { All flights to mainland China and South Korea; maintaining services to Hong } \\
\text { Kong and Taiwan }\end{array}$ \\
& Vietnam & $2 / 4 / 20$ & $\begin{array}{l}\text { Flights between Vietnam and Beijing, Shanghai, Guangzhou, Shenzhen, } \\
\text { Chengdu, Macau and South Korean destinations; between Hanoi and Hong }\end{array}$ \\
Vietnam Airlines & Kong; cut capacity to London, Paris, Frankfurt, Hong Kong and Seoul \\
Yemenia & $3 / 18 / 20$ & $\begin{array}{l}\text { Suspending all flights } \\
\text { Suspending all flights }\end{array}$ \\
FastJet Zimbabwe & Zimbabwe & $3 / 27 / 20$ &
\end{tabular}


Table S3: GISAID sequence IDs collected on April 25, 2020.

\begin{tabular}{l}
\hline ID \\
\hline \hline EPI_ISL_417541 \\
EPI_ISL_419448 \\
EPI_ISL_424900 \\
EPI_ISL_419301 \\
EPI_ISL_428832 \\
EPI_ISL_413850 \\
EPI_ISL_419738 \\
EPI_ISL_424528 \\
EPI_ISL_415529 \\
EPI_ISL_421922 \\
EPI_ISL_421900 \\
EPI_ISL_427334 \\
EPI_ISL_414562 \\
EPI_ISL_404227 \\
EPI_ISL_422032 \\
EPI_ISL_417234 \\
EPI_ISL_426763 \\
EPI_ISL_420731 \\
EPI_ISL_417386 \\
EPI_ISL_417267 \\
EPI_ISL_422888 \\
EPI_ISL_429882 \\
EPI_ISL_419824 \\
EPI_ISL_424227 \\
EPI_ISL_426307 \\
EPI_ISL_425762 \\
EPI_ISL_417813 \\
EPI_ISL_422602 \\
EPI_ISL_427208 \\
EPI_ISL_424092 \\
EPI_ISL_421934 \\
EPI_ISL_423205 \\
EPI_ISL_422019 \\
EPI_ISL_421746 \\
EPI_ISL_420003 \\
EPI_ISL_424215 \\
EPI_ISL_421411 \\
EPI_ISL_416718
\end{tabular}


Table S3: GISAID sequence IDs collected on April 25, 2020.

\begin{tabular}{l}
\hline ID \\
\hline \hline EPI_ISL_417669 \\
EPI_ISL_402129 \\
EPI_ISL_425857 \\
EPI_ISL_426978 \\
EPI_ISL_423952 \\
EPI_ISL_420773 \\
EPI_ISL_423332 \\
EPI_ISL_419436 \\
EPI_ISL_420691 \\
EPI_ISL_420516 \\
EPI_ISL_426689 \\
EPI_ISL_429483 \\
EPI_ISL_420174 \\
EPI_ISL_419674 \\
EPI_ISL_429022 \\
EPI_ISL_429089 \\
EPI_ISL_420569 \\
EPI_ISL_423642 \\
EPI_ISL_426852 \\
EPI_ISL_422042 \\
EPI_ISL_423384 \\
EPI_ISL_425872 \\
EPI_ISL_426979 \\
EPI_ISL_417466 \\
EPI_ISL_422071 \\
EPI_ISL_428319 \\
EPI_ISL_423221 \\
EPI_ISL_422421 \\
EPI_ISL_419907 \\
EPI_ISL_427381 \\
EPI_ISL_424498 \\
EPI_ISL_427764 \\
EPI_ISL_418433 \\
EPI_ISL_426642 \\
EPI_ISL_417348 \\
EPI_ISL_424379 \\
EPI_ISL_430097 \\
EPI_ISL_419582
\end{tabular}


Table S3: GISAID sequence IDs collected on April 25, 2020.

\begin{tabular}{l}
\hline ID \\
\hline \hline EPI_ISL_424482 \\
EPI_ISL_428262 \\
EPI_ISL_417092 \\
EPI_ISL_429131 \\
EPI_ISL_423161 \\
EPI_ISL_419585 \\
EPI_ISL_418885 \\
EPI_ISL_421314 \\
EPI_ISL_417332 \\
EPI_ISL_418823 \\
EPI_ISL_426941 \\
EPI_ISL_416589 \\
EPI_ISL_426085 \\
EPI_ISL_423310 \\
EPI_ISL_423375 \\
EPI_ISL_418290 \\
EPI_ISL_419935 \\
EPI_ISL_417878 \\
EPI_ISL_420478 \\
EPI_ISL_421879 \\
EPI_ISL_414526 \\
EPI_ISL_419902 \\
EPI_ISL_428272 \\
EPI_ISL_422977 \\
EPI_ISL_417181 \\
EPI_ISL_425048 \\
EPI_ISL_411952 \\
EPI_ISL_427497 \\
EPI_ISL_421427 \\
EPI_ISL_427199 \\
EPI_ISL_425596 \\
EPI_ISL_415532 \\
EPI_ISL_425238 \\
EPI_ISL_420114 \\
EPI_ISL_419532 \\
EPI_ISL_425056 \\
EPI_ISL_419712 \\
EPI_ISL_420612
\end{tabular}


Table S3: GISAID sequence IDs collected on April 25, 2020.

\begin{tabular}{l}
\hline ID \\
\hline \hline EPI_ISL_423000 \\
EPI_ISL_416711 \\
EPI_ISL_417514 \\
EPI_ISL_416482 \\
EPI_ISL_418641 \\
EPI_ISL_421479 \\
EPI_ISL_418033 \\
EPI_ISL_422081 \\
EPI_ISL_424032 \\
EPI_ISL_421004 \\
EPI_ISL_419265 \\
EPI_ISL_425694 \\
EPI_ISL_424476 \\
EPI_ISL_429155 \\
EPI_ISL_413853 \\
EPI_ISL_417524 \\
EPI_ISL_424050 \\
EPI_ISL_429067 \\
EPI_ISL_426729 \\
EPI_ISL_425242 \\
EPI_ISL_416365 \\
EPI_ISL_417301 \\
EPI_ISL_420847 \\
EPI_ISL_422330 \\
EPI_ISL_421260 \\
EPI_ISL_421630 \\
EPI_ISL_425206 \\
EPI_ISL_421738 \\
EPI_ISL_423474 \\
EPI_ISL_426954 \\
EPI_ISL_419559 \\
EPI_ISL_426774 \\
EPI_ISL_417663 \\
EPI_ISL_420749 \\
EPI_ISL_422889 \\
EPI_ISL_427488 \\
EPI_ISL_420477 \\
EPI_ISL_426811
\end{tabular}


Table S3: GISAID sequence IDs collected on April 25, 2020.

\begin{tabular}{l}
\hline ID \\
\hline \hline EPI_ISL_424221 \\
EPI_ISL_427263 \\
EPI_ISL_406531 \\
EPI_ISL_416699 \\
EPI_ISL_421617 \\
EPI_ISL_418896 \\
EPI_ISL_425383 \\
EPI_ISL_418967 \\
EPI_ISL_423477 \\
EPI_ISL_417690 \\
EPI_ISL_429162 \\
EPI_ISL_422927 \\
EPI_ISL_416676 \\
EPI_ISL_427160 \\
EPI_ISL_419499 \\
EPI_ISL_417937 \\
EPI_ISL_418894 \\
EPI_ISL_427088 \\
EPI_ISL_417224 \\
EPI_ISL_424317 \\
EPI_ISL_417850 \\
EPI_ISL_425535 \\
EPI_ISL_427247 \\
EPI_ISL_425688 \\
EPI_ISL_420360 \\
EPI_ISL_420549 \\
EPI_ISL_417222 \\
EPI_ISL_417007 \\
EPI_ISL_426092 \\
EPI_ISL_415538 \\
EPI_ISL_418931 \\
EPI_ISL_421388 \\
EPI_ISL_423135 \\
EPI_ISL_426965 \\
EPI_ISL_428233 \\
EPI_ISL_422397 \\
EPI_ISL_424546 \\
EPI_ISL_424642
\end{tabular}


Table S3: GISAID sequence IDs collected on April 25, 2020.

\begin{tabular}{l}
\hline ID \\
\hline \hline EPI_ISL_429491 \\
EPI_ISL_421691 \\
EPI_ISL_429782 \\
EPI_ISL_419561 \\
EPI_ISL_425988 \\
EPI_ISL_419936 \\
EPI_ISL_422939 \\
EPI_ISL_423638 \\
EPI_ISL_429531 \\
EPI_ISL_424301 \\
EPI_ISL_427290 \\
EPI_ISL_422913 \\
EPI_ISL_425904 \\
EPI_ISL_415595 \\
EPI_ISL_421002 \\
EPI_ISL_416444 \\
EPI_ISL_422128 \\
EPI_ISL_421560 \\
EPI_ISL_422527 \\
EPI_ISL_419749 \\
EPI_ISL_417122 \\
EPI_ISL_422567 \\
EPI_ISL_419834 \\
EPI_ISL_423435 \\
EPI_ISL_417144 \\
EPI_ISL_415743 \\
EPI_ISL_429504 \\
EPI_ISL_427549 \\
EPI_ISL_429744 \\
EPI_ISL_418965 \\
EPI_ISL_422667 \\
EPI_ISL_417163 \\
EPI_ISL_417350 \\
EPI_ISL_422839 \\
EPI_ISL_427773 \\
EPI_ISL_420236 \\
EPI_ISL_429717 \\
EPI_ISL_427744
\end{tabular}


Table S3: GISAID sequence IDs collected on April 25, 2020.

\begin{tabular}{l}
\hline ID \\
\hline \hline EPI_ISL_423968 \\
EPI_ISL_425362 \\
EPI_ISL_422747 \\
EPI_ISL_415650 \\
EPI_ISL_422917 \\
EPI_ISL_420346 \\
EPI_ISL_417022 \\
EPI_ISL_417364 \\
EPI_ISL_416450 \\
EPI_ISL_427474 \\
EPI_ISL_427353 \\
EPI_ISL_423096 \\
EPI_ISL_423063 \\
EPI_ISL_423125 \\
EPI_ISL_422949 \\
EPI_ISL_427110 \\
EPI_ISL_420527 \\
EPI_ISL_418388 \\
EPI_ISL_416698 \\
EPI_ISL_424609 \\
EPI_ISL_418770 \\
EPI_ISL_414486 \\
EPI_ISL_428254 \\
EPI_ISL_418192 \\
EPI_ISL_422880 \\
EPI_ISL_425055 \\
EPI_ISL_418114 \\
EPI_ISL_423854 \\
EPI_ISL_427787 \\
EPI_ISL_426502 \\
EPI_ISL_429281 \\
EPI_ISL_425820 \\
EPI_ISL_419173 \\
EPI_ISL_430021 \\
EPI_ISL_425682 \\
EPI_ISL_420585 \\
EPI_ISL_413653 \\
EPI_ISL_430070
\end{tabular}


Table S3: GISAID sequence IDs collected on April 25, 2020.

\begin{tabular}{l}
\hline ID \\
\hline \hline EPI_ISL_424342 \\
EPI_ISL_426306 \\
EPI_ISL_425430 \\
EPI_ISL_424574 \\
EPI_ISL_421289 \\
EPI_ISL_420611 \\
EPI_ISL_414366 \\
EPI_ISL_426617 \\
EPI_ISL_421207 \\
EPI_ISL_420532 \\
EPI_ISL_418294 \\
EPI_ISL_419832 \\
EPI_ISL_421281 \\
EPI_ISL_417361 \\
EPI_ISL_423176 \\
EPI_ISL_417641 \\
EPI_ISL_424499 \\
EPI_ISL_414621 \\
EPI_ISL_423379 \\
EPI_ISL_418971 \\
EPI_ISL_426993 \\
EPI_ISL_428757 \\
EPI_ISL_419460 \\
EPI_ISL_424272 \\
EPI_ISL_414043 \\
EPI_ISL_428468 \\
EPI_ISL_428232 \\
EPI_ISL_418260 \\
EPI_ISL_419568 \\
EPI_ISL_429564 \\
EPI_ISL_421473 \\
EPI_ISL_427365 \\
EPI_ISL_423823 \\
EPI_ISL_425951 \\
EPI_ISL_424354 \\
EPI_ISL_424103 \\
EPI_ISL_424960 \\
EPI_ISL_417584
\end{tabular}


Table S3: GISAID sequence IDs collected on April 25, 2020.

\begin{tabular}{l}
\hline ID \\
\hline \hline EPI_ISL_422323 \\
EPI_ISL_420058 \\
EPI_ISL_420208 \\
EPI_ISL_423124 \\
EPI_ISL_427084 \\
EPI_ISL_425673 \\
EPI_ISL_417481 \\
EPI_ISL_423074 \\
EPI_ISL_417536 \\
EPI_ISL_420248 \\
EPI_ISL_420209 \\
EPI_ISL_413607 \\
EPI_ISL_420483 \\
EPI_ISL_429359 \\
EPI_ISL_423936 \\
EPI_ISL_417193 \\
EPI_ISL_417477 \\
EPI_ISL_418834 \\
EPI_ISL_423560 \\
EPI_ISL_416488 \\
EPI_ISL_424449 \\
EPI_ISL_417246 \\
EPI_ISL_425521 \\
EPI_ISL_427555 \\
EPI_ISL_424162 \\
EPI_ISL_425135 \\
EPI_ISL_412898 \\
EPI_ISL_421855 \\
EPI_ISL_422303 \\
EPI_ISL_429464 \\
EPI_ISL_426555 \\
EPI_ISL_426964 \\
EPI_ISL_418796 \\
EPI_ISL_418240 \\
EPI_ISL_420461 \\
EPI_ISL_429969 \\
EPI_ISL_416368 \\
EPI_ISL_427205
\end{tabular}


Table S3: GISAID sequence IDs collected on April 25, 2020.

\begin{tabular}{l}
\hline ID \\
\hline \hline EPI_ISL_426685 \\
EPI_ISL_426014 \\
EPI_ISL_418218 \\
EPI_ISL_413858 \\
EPI_ISL_427786 \\
EPI_ISL_420798 \\
EPI_ISL_424956 \\
EPI_ISL_419984 \\
EPI_ISL_419899 \\
EPI_ISL_425984 \\
EPI_ISL_429485 \\
EPI_ISL_429282 \\
EPI_ISL_421291 \\
EPI_ISL_415710 \\
EPI_ISL_429467 \\
EPI_ISL_418346 \\
EPI_ISL_425913 \\
EPI_ISL_414633 \\
EPI_ISL_420460 \\
EPI_ISL_429097 \\
EPI_ISL_415479 \\
EPI_ISL_429142 \\
EPI_ISL_417816 \\
EPI_ISL_417836 \\
EPI_ISL_414539 \\
EPI_ISL_426288 \\
EPI_ISL_416427 \\
EPI_ISL_418652 \\
EPI_ISL_425891 \\
EPI_ISL_422651 \\
EPI_ISL_429082 \\
EPI_ISL_420631 \\
EPI_ISL_419564 \\
EPI_ISL_421355 \\
EPI_ISL_421450 \\
EPI_ISL_426471 \\
EPI_ISL_407976 \\
EPI_ISL_418284
\end{tabular}


Table S3: GISAID sequence IDs collected on April 25, 2020.

\begin{tabular}{l}
\hline ID \\
\hline \hline EPI_ISL_428373 \\
EPI_ISL_427789 \\
EPI_ISL_427650 \\
EPI_ISL_420120 \\
EPI_ISL_429511 \\
EPI_ISL_419584 \\
EPI_ISL_424880 \\
EPI_ISL_419720 \\
EPI_ISL_429305 \\
EPI_ISL_414586 \\
EPI_ISL_421417 \\
EPI_ISL_422427 \\
EPI_ISL_418646 \\
EPI_ISL_418159 \\
EPI_ISL_428359 \\
EPI_ISL_423347 \\
EPI_ISL_422871 \\
EPI_ISL_419767 \\
EPI_ISL_426527 \\
EPI_ISL_426564 \\
EPI_ISL_421755 \\
EPI_ISL_418155 \\
EPI_ISL_427034 \\
EPI_ISL_426750 \\
EPI_ISL_420145 \\
EPI_ISL_418826 \\
EPI_ISL_426820 \\
EPI_ISL_428253 \\
EPI_ISL_427017 \\
EPI_ISL_417313 \\
EPI_ISL_420930 \\
EPI_ISL_414564 \\
EPI_ISL_420137 \\
EPI_ISL_419960 \\
EPI_ISL_429065 \\
EPI_ISL_417795 \\
EPI_ISL_416491 \\
EPI_ISL_426082
\end{tabular}


Table S3: GISAID sequence IDs collected on April 25, 2020.

\begin{tabular}{l}
\hline ID \\
\hline \hline EPI_ISL_420200 \\
EPI_ISL_429561 \\
EPI_ISL_423358 \\
EPI_ISL_423212 \\
EPI_ISL_419218 \\
EPI_ISL_425170 \\
EPI_ISL_419524 \\
EPI_ISL_421683 \\
EPI_ISL_427073 \\
EPI_ISL_415977 \\
EPI_ISL_417638 \\
EPI_ISL_429622 \\
EPI_ISL_423592 \\
EPI_ISL_429607 \\
EPI_ISL_419971 \\
EPI_ISL_424065 \\
EPI_ISL_422938 \\
EPI_ISL_418188 \\
EPI_ISL_418045 \\
EPI_ISL_427324 \\
EPI_ISL_425187 \\
EPI_ISL_426567 \\
EPI_ISL_418712 \\
EPI_ISL_421573 \\
EPI_ISL_426091 \\
EPI_ISL_414438 \\
EPI_ISL_419254 \\
EPI_ISL_430019 \\
EPI_ISL_425609 \\
EPI_ISL_422076 \\
EPI_ISL_419482 \\
EPI_ISL_417854 \\
EPI_ISL_419848 \\
EPI_ISL_429015 \\
EPI_ISL_421729 \\
EPI_ISL_424262 \\
EPI_ISL_416524 \\
EPI_ISL_424008
\end{tabular}


Table S3: GISAID sequence IDs collected on April 25, 2020.

\begin{tabular}{l}
\hline ID \\
\hline \hline EPI_ISL_428325 \\
EPI_ISL_421243 \\
EPI_ISL_418720 \\
EPI_ISL_413997 \\
EPI_ISL_417560 \\
EPI_ISL_423044 \\
EPI_ISL_425287 \\
EPI_ISL_427674 \\
EPI_ISL_423122 \\
EPI_ISL_417194 \\
EPI_ISL_422057 \\
EPI_ISL_416721 \\
EPI_ISL_416744 \\
EPI_ISL_429346 \\
EPI_ISL_428347 \\
EPI_ISL_429465 \\
EPI_ISL_428900 \\
EPI_ISL_426074 \\
EPI_ISL_416334 \\
EPI_ISL_423692 \\
EPI_ISL_428800 \\
EPI_ISL_418665 \\
EPI_ISL_425470 \\
EPI_ISL_422746 \\
EPI_ISL_417004 \\
EPI_ISL_419517 \\
EPI_ISL_427531 \\
EPI_ISL_424938 \\
EPI_ISL_423027 \\
EPI_ISL_425784 \\
EPI_ISL_426768 \\
EPI_ISL_424937 \\
EPI_ISL_425511 \\
EPI_ISL_414692 \\
EPI_ISL_428267 \\
EPI_ISL_417426 \\
EPI_ISL_420040 \\
EPI_ISL_418220
\end{tabular}


Table S3: GISAID sequence IDs collected on April 25, 2020.

\begin{tabular}{l}
\hline ID \\
\hline \hline EPI_ISL_422164 \\
EPI_ISL_411950 \\
EPI_ISL_420078 \\
EPI_ISL_421743 \\
EPI_ISL_429884 \\
EPI_ISL_422694 \\
EPI_ISL_420326 \\
EPI_ISL_425720 \\
EPI_ISL_419260 \\
EPI_ISL_422557 \\
EPI_ISL_421432 \\
EPI_ISL_422944 \\
EPI_ISL_417675 \\
EPI_ISL_424059 \\
EPI_ISL_418869 \\
EPI_ISL_418882 \\
EPI_ISL_422222 \\
EPI_ISL_424974 \\
EPI_ISL_427545 \\
EPI_ISL_423216 \\
EPI_ISL_418739 \\
EPI_ISL_423856 \\
EPI_ISL_429634 \\
EPI_ISL_421320 \\
EPI_ISL_427455 \\
EPI_ISL_427071 \\
EPI_ISL_429565 \\
EPI_ISL_424857 \\
EPI_ISL_427164 \\
EPI_ISL_422174 \\
EPI_ISL_422115 \\
EPI_ISL_423941 \\
EPI_ISL_422855 \\
EPI_ISL_426059 \\
EPI_ISL_424846 \\
EPI_ISL_418016 \\
EPI_ISL_427106 \\
EPI_ISL_424209
\end{tabular}


Table S3: GISAID sequence IDs collected on April 25, 2020.

\begin{tabular}{l}
\hline ID \\
\hline \hline EPI_ISL_417296 \\
EPI_ISL_420570 \\
EPI_ISL_424186 \\
EPI_ISL_426460 \\
EPI_ISL_426653 \\
EPI_ISL_428878 \\
EPI_ISL_420842 \\
EPI_ISL_425455 \\
EPI_ISL_423320 \\
EPI_ISL_420305 \\
EPI_ISL_418768 \\
EPI_ISL_418935 \\
EPI_ISL_426164 \\
EPI_ISL_427692 \\
EPI_ISL_418717 \\
EPI_ISL_425923 \\
EPI_ISL_422317 \\
EPI_ISL_420505 \\
EPI_ISL_416737 \\
EPI_ISL_429583 \\
EPI_ISL_416694 \\
EPI_ISL_425053 \\
EPI_ISL_421430 \\
EPI_ISL_429008 \\
EPI_ISL_423817 \\
EPI_ISL_406970 \\
EPI_ISL_421612 \\
EPI_ISL_415602 \\
EPI_ISL_427127 \\
EPI_ISL_414550 \\
EPI_ISL_417270 \\
EPI_ISL_429558 \\
EPI_ISL_424606 \\
EPI_ISL_429569 \\
EPI_ISL_424521 \\
EPI_ISL_413652 \\
EPI_ISL_423596 \\
EPI_ISL_423663
\end{tabular}


Table S3: GISAID sequence IDs collected on April 25, 2020.

\begin{tabular}{l}
\hline ID \\
\hline \hline EPI_ISL_419417 \\
EPI_ISL_419589 \\
EPI_ISL_420117 \\
EPI_ISL_422767 \\
EPI_ISL_426695 \\
EPI_ISL_420010 \\
EPI_ISL_415507 \\
EPI_ISL_421847 \\
EPI_ISL_426683 \\
EPI_ISL_422849 \\
EPI_ISL_426928 \\
EPI_ISL_429631 \\
EPI_ISL_420511 \\
EPI_ISL_429643 \\
EPI_ISL_423639 \\
EPI_ISL_420670 \\
EPI_ISL_421909 \\
EPI_ISL_421453 \\
EPI_ISL_427663 \\
EPI_ISL_420224 \\
EPI_ISL_421311 \\
EPI_ISL_413518 \\
EPI_ISL_421705 \\
EPI_ISL_424041 \\
EPI_ISL_419506 \\
EPI_ISL_414471 \\
EPI_ISL_423693 \\
EPI_ISL_417655 \\
EPI_ISL_422520 \\
EPI_ISL_423195 \\
EPI_ISL_416326 \\
EPI_ISL_425786 \\
EPI_ISL_416584 \\
EPI_ISL_423871 \\
EPI_ISL_414534 \\
EPI_ISL_427063 \\
EPI_ISL_423109 \\
EPI_ISL_420129
\end{tabular}


Table S3: GISAID sequence IDs collected on April 25, 2020.

\begin{tabular}{l}
\hline ID \\
\hline \hline EPI_ISL_423484 \\
EPI_ISL_420775 \\
EPI_ISL_429121 \\
EPI_ISL_418337 \\
EPI_ISL_424406 \\
EPI_ISL_419823 \\
EPI_ISL_426624 \\
EPI_ISL_416693 \\
EPI_ISL_420267 \\
EPI_ISL_423507 \\
EPI_ISL_415148 \\
EPI_ISL_414456 \\
EPI_ISL_423406 \\
EPI_ISL_421924 \\
EPI_ISL_422940 \\
EPI_ISL_429119 \\
EPI_ISL_420020 \\
EPI_ISL_419533 \\
EPI_ISL_423584 \\
EPI_ISL_421198 \\
EPI_ISL_420622 \\
EPI_ISL_429397 \\
EPI_ISL_426982 \\
EPI_ISL_419835 \\
EPI_ISL_421984 \\
EPI_ISL_426116 \\
EPI_ISL_428478 \\
EPI_ISL_420393 \\
EPI_ISL_419751 \\
EPI_ISL_424579 \\
EPI_ISL_420053 \\
EPI_ISL_423314 \\
EPI_ISL_421828 \\
EPI_ISL_422504 \\
EPI_ISL_422893 \\
EPI_ISL_416690 \\
EPI_ISL_424963 \\
EPI_ISL_426113
\end{tabular}


Table S3: GISAID sequence IDs collected on April 25, 2020.

\begin{tabular}{l}
\hline ID \\
\hline \hline EPI_ISL_421946 \\
EPI_ISL_427484 \\
EPI_ISL_416315 \\
EPI_ISL_422118 \\
EPI_ISL_423030 \\
EPI_ISL_426066 \\
EPI_ISL_421970 \\
EPI_ISL_426918 \\
EPI_ISL_423415 \\
EPI_ISL_421622 \\
EPI_ISL_427448 \\
EPI_ISL_429866 \\
EPI_ISL_426558 \\
EPI_ISL_421368 \\
EPI_ISL_418027 \\
EPI_ISL_416448 \\
EPI_ISL_417260 \\
EPI_ISL_427308 \\
EPI_ISL_421393 \\
EPI_ISL_420009 \\
EPI_ISL_423885 \\
EPI_ISL_420276 \\
EPI_ISL_428927 \\
EPI_ISL_425529 \\
EPI_ISL_420553 \\
EPI_ISL_418925 \\
EPI_ISL_423746 \\
EPI_ISL_422730 \\
EPI_ISL_426985 \\
EPI_ISL_425361 \\
EPI_ISL_427366 \\
EPI_ISL_418232 \\
EPI_ISL_425471 \\
EPI_ISL_421421 \\
EPI_ISL_428288 \\
EPI_ISL_421309 \\
EPI_ISL_416432 \\
EPI_ISL_416758
\end{tabular}


Table S3: GISAID sequence IDs collected on April 25, 2020.

\begin{tabular}{l}
\hline ID \\
\hline \hline EPI_ISL_423904 \\
EPI_ISL_426694 \\
EPI_ISL_425241 \\
EPI_ISL_427068 \\
EPI_ISL_424308 \\
EPI_ISL_426506 \\
EPI_ISL_426068 \\
EPI_ISL_423764 \\
EPI_ISL_428352 \\
EPI_ISL_423360 \\
EPI_ISL_421303 \\
EPI_ISL_421390 \\
EPI_ISL_429629 \\
EPI_ISL_426929 \\
EPI_ISL_420214 \\
EPI_ISL_416585 \\
EPI_ISL_419233 \\
EPI_ISL_418981 \\
EPI_ISL_422157 \\
EPI_ISL_425138 \\
EPI_ISL_425301 \\
EPI_ISL_415515 \\
EPI_ISL_421350 \\
EPI_ISL_420630 \\
EPI_ISL_418212 \\
EPI_ISL_429800 \\
EPI_ISL_429290 \\
EPI_ISL_415613 \\
EPI_ISL_415658 \\
EPI_ISL_423497 \\
EPI_ISL_408977 \\
EPI_ISL_417630 \\
EPI_ISL_429624 \\
EPI_ISL_424143 \\
EPI_ISL_420485 \\
EPI_ISL_413020 \\
EPI_ISL_423165 \\
EPI_ISL_425708
\end{tabular}


Table S3: GISAID sequence IDs collected on April 25, 2020.

\begin{tabular}{l}
\hline ID \\
\hline \hline EPI_ISL_420496 \\
EPI_ISL_427317 \\
EPI_ISL_423412 \\
EPI_ISL_423075 \\
EPI_ISL_424038 \\
EPI_ISL_416437 \\
EPI_ISL_417133 \\
EPI_ISL_429219 \\
EPI_ISL_429530 \\
EPI_ISL_429737 \\
EPI_ISL_426807 \\
EPI_ISL_425630 \\
EPI_ISL_423211 \\
EPI_ISL_422320 \\
EPI_ISL_424550 \\
EPI_ISL_426955 \\
EPI_ISL_413615 \\
EPI_ISL_423503 \\
EPI_ISL_417973 \\
EPI_ISL_419976 \\
EPI_ISL_421994 \\
EPI_ISL_425982 \\
EPI_ISL_429458 \\
EPI_ISL_425568 \\
EPI_ISL_424864 \\
EPI_ISL_418508 \\
EPI_ISL_417855 \\
EPI_ISL_423126 \\
EPI_ISL_420656 \\
EPI_ISL_423868 \\
EPI_ISL_425600 \\
EPI_ISL_429099 \\
EPI_ISL_418072 \\
EPI_ISL_429038 \\
EPI_ISL_417470 \\
EPI_ISL_423923 \\
EPI_ISL_425133 \\
EPI_ISL_426560
\end{tabular}


Table S3: GISAID sequence IDs collected on April 25, 2020.

\begin{tabular}{l}
\hline ID \\
\hline \hline EPI_ISL_429517 \\
EPI_ISL_423879 \\
EPI_ISL_417099 \\
EPI_ISL_420685 \\
EPI_ISL_429599 \\
EPI_ISL_423397 \\
EPI_ISL_417876 \\
EPI_ISL_423667 \\
EPI_ISL_419892 \\
EPI_ISL_429080 \\
EPI_ISL_429079 \\
EPI_ISL_418956 \\
EPI_ISL_420086 \\
EPI_ISL_417472 \\
EPI_ISL_427351 \\
EPI_ISL_419593 \\
EPI_ISL_420935 \\
EPI_ISL_428836 \\
EPI_ISL_417544 \\
EPI_ISL_416594 \\
EPI_ISL_417405 \\
EPI_ISL_425862 \\
EPI_ISL_421829 \\
EPI_ISL_425712 \\
EPI_ISL_427103 \\
EPI_ISL_423716 \\
EPI_ISL_414007 \\
EPI_ISL_419558 \\
EPI_ISL_428960 \\
EPI_ISL_416517 \\
EPI_ISL_423429 \\
EPI_ISL_422697 \\
EPI_ISL_420969 \\
EPI_ISL_426734 \\
EPI_ISL_430093 \\
EPI_ISL_426035 \\
EPI_ISL_420512 \\
EPI_ISL_419587
\end{tabular}


Table S3: GISAID sequence IDs collected on April 25, 2020.

\begin{tabular}{l}
\hline ID \\
\hline \hline EPI_ISL_427204 \\
EPI_ISL_417359 \\
EPI_ISL_416581 \\
EPI_ISL_417796 \\
EPI_ISL_415142 \\
EPI_ISL_419684 \\
EPI_ISL_419741 \\
EPI_ISL_416596 \\
EPI_ISL_425401 \\
EPI_ISL_427699 \\
EPI_ISL_422167 \\
EPI_ISL_429299 \\
EPI_ISL_415707 \\
EPI_ISL_423336 \\
EPI_ISL_428670 \\
EPI_ISL_427500 \\
EPI_ISL_425177 \\
EPI_ISL_422603 \\
EPI_ISL_424509 \\
EPI_ISL_419993 \\
EPI_ISL_428709 \\
EPI_ISL_428284 \\
EPI_ISL_421902 \\
EPI_ISL_419943 \\
EPI_ISL_421244 \\
EPI_ISL_419927 \\
EPI_ISL_421720 \\
EPI_ISL_419223 \\
EPI_ISL_418963 \\
EPI_ISL_426289 \\
EPI_ISL_426724 \\
EPI_ISL_424146 \\
EPI_ISL_421776 \\
EPI_ISL_425182 \\
EPI_ISL_414517 \\
EPI_ISL_416443 \\
EPI_ISL_414541 \\
EPI_ISL_422689
\end{tabular}


Table S3: GISAID sequence IDs collected on April 25, 2020.

\begin{tabular}{l}
\hline ID \\
\hline \hline EPI_ISL_417742 \\
EPI_ISL_418015 \\
EPI_ISL_421996 \\
EPI_ISL_414643 \\
EPI_ISL_428317 \\
EPI_ISL_422345 \\
EPI_ISL_420521 \\
EPI_ISL_420318 \\
EPI_ISL_424431 \\
EPI_ISL_422265 \\
EPI_ISL_418293 \\
EPI_ISL_422432 \\
EPI_ISL_424197 \\
EPI_ISL_425178 \\
EPI_ISL_420940 \\
EPI_ISL_417432 \\
EPI_ISL_425692 \\
EPI_ISL_417444 \\
EPI_ISL_429821 \\
EPI_ISL_420226 \\
EPI_ISL_414542 \\
EPI_ISL_418025 \\
EPI_ISL_429010 \\
EPI_ISL_425122 \\
EPI_ISL_421301 \\
EPI_ISL_423957 \\
EPI_ISL_423949 \\
EPI_ISL_428870 \\
EPI_ISL_420154 \\
EPI_ISL_422941 \\
EPI_ISL_423246 \\
EPI_ISL_421876 \\
EPI_ISL_430100 \\
EPI_ISL_426075 \\
EPI_ISL_417215 \\
EPI_ISL_419228 \\
EPI_ISL_418777 \\
EPI_ISL_418953
\end{tabular}


Table S3: GISAID sequence IDs collected on April 25, 2020.

\begin{tabular}{l}
\hline ID \\
\hline \hline EPI_ISL_418920 \\
EPI_ISL_430020 \\
EPI_ISL_424865 \\
EPI_ISL_423054 \\
EPI_ISL_423175 \\
EPI_ISL_427123 \\
EPI_ISL_425953 \\
EPI_ISL_416499 \\
EPI_ISL_429209 \\
EPI_ISL_427081 \\
EPI_ISL_430001 \\
EPI_ISL_423307 \\
EPI_ISL_417204 \\
EPI_ISL_427629 \\
EPI_ISL_420250 \\
EPI_ISL_423010 \\
EPI_ISL_429603 \\
EPI_ISL_424643 \\
EPI_ISL_408479 \\
EPI_ISL_425941 \\
EPI_ISL_423189 \\
EPI_ISL_425449 \\
EPI_ISL_424874 \\
EPI_ISL_416753 \\
EPI_ISL_418994 \\
EPI_ISL_425944 \\
EPI_ISL_423586 \\
EPI_ISL_413589 \\
EPI_ISL_417575 \\
EPI_ISL_430028 \\
EPI_ISL_423803 \\
EPI_ISL_427129 \\
EPI_ISL_418753 \\
EPI_ISL_429853 \\
EPI_ISL_428395 \\
EPI_ISL_417564 \\
EPI_ISL_420985 \\
EPI_ISL_420382
\end{tabular}


Table S3: GISAID sequence IDs collected on April 25, 2020.

\begin{tabular}{l}
\hline ID \\
\hline \hline EPI_ISL_425691 \\
EPI_ISL_427700 \\
EPI_ISL_427065 \\
EPI_ISL_427304 \\
EPI_ISL_416607 \\
EPI_ISL_420321 \\
EPI_ISL_426064 \\
EPI_ISL_421395 \\
EPI_ISL_422253 \\
EPI_ISL_427452 \\
EPI_ISL_423494 \\
EPI_ISL_422422 \\
EPI_ISL_417569 \\
EPI_ISL_418044 \\
EPI_ISL_424128 \\
EPI_ISL_421382 \\
EPI_ISL_422542 \\
EPI_ISL_424176 \\
EPI_ISL_426499 \\
EPI_ISL_424466 \\
EPI_ISL_425358 \\
EPI_ISL_420195 \\
EPI_ISL_424895 \\
EPI_ISL_424414 \\
EPI_ISL_413569 \\
EPI_ISL_424506 \\
EPI_ISL_427566 \\
EPI_ISL_422529 \\
EPI_ISL_403935 \\
EPI_ISL_423637 \\
EPI_ISL_420007 \\
EPI_ISL_412981 \\
EPI_ISL_424397 \\
EPI_ISL_426550 \\
EPI_ISL_417024 \\
EPI_ISL_427385 \\
EPI_ISL_422054 \\
EPI_ISL_415461
\end{tabular}


Table S3: GISAID sequence IDs collected on April 25, 2020.

\begin{tabular}{l}
\hline ID \\
\hline \hline EPI_ISL_426937 \\
EPI_ISL_426519 \\
EPI_ISL_427252 \\
EPI_ISL_428334 \\
EPI_ISL_418695 \\
EPI_ISL_428794 \\
EPI_ISL_429183 \\
EPI_ISL_424384 \\
EPI_ISL_427069 \\
EPI_ISL_425167 \\
EPI_ISL_419724 \\
EPI_ISL_416331 \\
EPI_ISL_423961 \\
EPI_ISL_427187 \\
EPI_ISL_424529 \\
EPI_ISL_424440 \\
EPI_ISL_419800 \\
EPI_ISL_425775 \\
EPI_ISL_425992 \\
EPI_ISL_420016 \\
EPI_ISL_416691 \\
EPI_ISL_427175 \\
EPI_ISL_426651 \\
EPI_ISL_428725 \\
EPI_ISL_418351 \\
EPI_ISL_423006 \\
EPI_ISL_423294 \\
EPI_ISL_421507 \\
EPI_ISL_424451 \\
EPI_ISL_429567 \\
EPI_ISL_426956 \\
EPI_ISL_428919 \\
EPI_ISL_419400 \\
EPI_ISL_420993 \\
EPI_ISL_421398 \\
EPI_ISL_429269 \\
EPI_ISL_419663 \\
EPI_ISL_417368
\end{tabular}


bioRxiv preprint doi: https://doi.org/10.1101/2020 11.06.370999; this version posted November 6, 2020. The copyright holder for this preprint (which was not certified by peer review) is the author/funder, who has granted bioRxiv a license to display the preprint in perpetuity. It is made available under aCC-BY 4.0 International license.

Table S3: GISAID sequence IDs collected on April 25, 2020.

\begin{tabular}{l}
\hline ID \\
\hline \hline EPI_ISL_428697 \\
EPI_ISL_427191 \\
EPI_ISL_423376 \\
EPI_ISL_428384 \\
EPI_ISL_425789 \\
EPI_ISL_417988 \\
EPI_ISL_422570 \\
EPI_ISL_427022 \\
EPI_ISL_422820 \\
EPI_ISL_414689 \\
EPI_ISL_416033 \\
EPI_ISL_417734 \\
EPI_ISL_425523 \\
\hline
\end{tabular}


Table S4: Clusters identified using a genetic distance threshold within $1 \%$ of the distribution of patristic distances within the entire tree. The minimum percentile threshold that maximized the number of clusters was chosen as the optimal threshold by performing multiple clustering runs on randomly sampled patristic distance distributions (1 million for each run) in Phylopart v2 (46).

\begin{tabular}{|c|c|c|c|c|c|}
\hline clustername & bootstrap & leafname & branchPath & medianOfDistances & sequencesperCluster \\
\hline 923 & 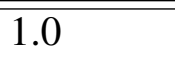 & "EPI_ISL_426132 & 6.893599999999978E-4 & 6.539999999999758E-6 & $\overline{4}$ \\
\hline 923 & 1.0 & EPI_ISL_429638 & 6.871799999999978E-4 & 6.539999999999758E-6 & 4 \\
\hline 923 & 1.0 & EPI_ISL_429626 & 6.893599999999978E-4 & 6.539999999999758E-6 & 4 \\
\hline 923 & 1.0 & EPI_ISL_429616 & 6.893599999999978E-4 & 6.539999999999758E-6 & 4 \\
\hline 1132 & 1.0 & EPI_ISL_418361 & 6.26129999999999E-4 & 4.359999999999911E-6 & 2 \\
\hline 1132 & 1.0 & EPI_ISL_425257 & 6.26129999999999E-4 & 4.359999999999911E-6 & 2 \\
\hline 1139 & 1.0 & EPI_ISL_424496 & 6.64909999999999E-4 & 3.877999999999998E-5 & 2 \\
\hline 1139 & 1.0 & EPI_ISL_422650 & 6.30489999999999E-4 & 3.877999999999998E-5 & 2 \\
\hline 1148 & 1.0 & EPI_ISL_419720 & 6.195899999999992E-4 & 1.7439999999999643E-5 & 12 \\
\hline 1148 & 1.0 & EPI_ISL_426804 & 6.28309999999999E-4 & 1.7439999999999643E-5 & 12 \\
\hline 1148 & 1.0 & EPI_ISL_419989 & $6.28309999999999 \mathrm{E}-4$ & 1.7439999999999643E-5 & 12 \\
\hline 1148 & 1.0 & EPI_ISL_427032 & 6.535599999999993E-4 & 1.7439999999999643E-5 & 12 \\
\hline 1148 & 1.0 & EPI_ISL_419911 & 6.28309999999999E-4 & 1.7439999999999643E-5 & 12 \\
\hline 1148 & 1.0 & EPI_ISL_419983 & 6.28309999999999E-4 & 1.7439999999999643E-5 & 12 \\
\hline 1148 & 1.0 & EPI_ISL_419997 & 6.239499999999991E-4 & 1.7439999999999643E-5 & 12 \\
\hline 1148 & 1.0 & EPI_ISL_419822 & 6.28309999999999E-4 & 1.7439999999999643E-5 & 12 \\
\hline 1148 & 1.0 & EPI_ISL_426807 & 6.152299999999993E-4 & 1.7439999999999643E-5 & 12 \\
\hline 1148 & 1.0 & EPI_ISL_419999 & 6.977599999999991E-4 & 1.7439999999999643E-5 & 12 \\
\hline 1148 & 1.0 & EPI_ISL_427156 & 6.566499999999992E-4 & 1.7439999999999643E-5 & 12 \\
\hline 1148 & 1.0 & EPI_ISL_419852 & 6.28309999999999E-4 & 1.7439999999999643E-5 & 12 \\
\hline 1202 & 1.0 & EPI_ISL_414635 & 6.64899999999999E-4 & 7.814999999999992E-5 & 3 \\
\hline 1202 & 1.0 & EPI_ISL_418239 & 7.38689999999999E-4 & 7.814999999999992E-5 & 3 \\
\hline 1202 & 1.0 & EPI_ISL_414636 & 7.38689999999999E-4 & 7.814999999999992E-5 & 3 \\
\hline 1241 & 1.0 & EPI_ISL_426467 & 6.785999999999987E-4 & $3.877000000000004 \mathrm{E}-5$ & 3 \\
\hline 1241 & 1.0 & EPI_ISL_426466 & 6.785999999999987E-4 & $3.877000000000004 \mathrm{E}-5$ & 3 \\
\hline 1241 & 1.0 & EPI_ISL_426457 & 7.130099999999987E-4 & $3.877000000000004 \mathrm{E}-5$ & 3 \\
\hline
\end{tabular}


Table S4: Clusters identified using a genetic distance threshold within $1 \%$ of the distribution of patristic distances within the entire tree. The minimum percentile threshold that maximized the number of clusters was chosen as the optimal threshold by performing multiple clustering runs on randomly sampled patristic distance distributions (1 million for each run) in Phylopart v2 (46).

\begin{tabular}{|c|c|c|c|c|c|}
\hline clustername & bootstrap & leafname & branchPath & medianOfDistances & sequencesperCluster \\
\hline 1246 & 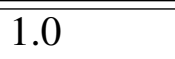 & "EPI_ISL_422047 & 6.785999999999987E-4 & $4.359999999999911 \mathrm{E}-6$ & 2 \\
\hline 1246 & 1.0 & EPI_ISL_422304 & 6.785999999999987E-4 & 4.359999999999911E-6 & 2 \\
\hline 1271 & 1.0 & EPI_ISL_420021 & 6.501099999999987E-4 & 4.775999999999977E-5 & 11 \\
\hline 1271 & 1.0 & EPI_ISL_420019 & 7.089799999999988E-4 & 4.775999999999977E-5 & 11 \\
\hline 1271 & 1.0 & EPI_ISL_420630 & 6.501099999999987E-4 & 4.775999999999977E-5 & 11 \\
\hline 1271 & 1.0 & EPI_ISL_420022 & 6.845199999999987E-4 & 4.775999999999977E-5 & 11 \\
\hline 1271 & 1.0 & EPI_ISL_420629 & 6.501099999999987E-4 & 4.775999999999977E-5 & 11 \\
\hline 1271 & 1.0 & EPI_ISL_420020 & 6.501099999999987E-4 & 4.775999999999977E-5 & 11 \\
\hline 1271 & 1.0 & EPI_ISL_419258 & 6.479299999999987E-4 & 4.775999999999977E-5 & 11 \\
\hline 1271 & 1.0 & EPI_ISL_429981 & 6.738899999999988E-4 & 4.775999999999977E-5 & 11 \\
\hline 1271 & 1.0 & EPI_ISL_419257 & 6.804799999999988E-4 & 4.775999999999977E-5 & 11 \\
\hline 1271 & 1.0 & EPI_ISL_429982 & 7.487899999999987E-4 & 4.775999999999977E-5 & 11 \\
\hline 1271 & 1.0 & EPI_ISL_426455 & 6.501099999999987E-4 & 4.775999999999977E-5 & 11 \\
\hline 1294 & 1.0 & EPI_ISL_429554 & 7.036999999999989E-4 & 7.322999999999987E-5 & 2 \\
\hline 1294 & 1.0 & EPI_ISL_429528 & 7.03709999999999E-4 & 7.322999999999987E-5 & 2 \\
\hline 1331 & 1.0 & EPI_ISL_422212 & 6.26129999999999E-4 & 4.531999999999995E-5 & 5 \\
\hline 1331 & 1.0 & EPI_ISL_422224 & 6.67089999999999E-4 & 4.531999999999995E-5 & 5 \\
\hline 1331 & 1.0 & EPI_ISL_428912 & 6.62719999999999E-4 & 4.531999999999995E-5 & 5 \\
\hline 1331 & 1.0 & EPI_ISL_422024 & 6.67089999999999E-4 & 4.531999999999995E-5 & 5 \\
\hline 1331 & 1.0 & EPI_ISL_422216 & 6.64909999999999E-4 & 4.531999999999995E-5 & 5 \\
\hline 1367 & 1.0 & EPI_ISL_421979 & 7.601999999999987E-4 & 6.539999999999758E-6 & 3 \\
\hline 1367 & 1.0 & EPI_ISL_420529 & 7.623799999999987E-4 & 6.539999999999758E-6 & 3 \\
\hline 1367 & 1.0 & EPI_ISL_420702 & 7.623799999999987E-4 & 6.539999999999758E-6 & 3 \\
\hline 1373 & 1.0 & EPI_ISL_428379 & 6.451899999999987E-4 & 3.921999999999997E-5 & 2 \\
\hline 1373 & 1.0 & EPI_ISL_419516 & 6.800499999999988E-4 & 3.921999999999997E-5 & 2 \\
\hline 1380 & 1.0 & EPI_ISL_428202 & 6.69019999999999E-4 & 7.450999999999985E-5 & 2 \\
\hline
\end{tabular}


Table S4: Clusters identified using a genetic distance threshold within $1 \%$ of the distribution of patristic distances within the entire tree. The minimum percentile threshold that maximized the number of clusters was chosen as the optimal threshold by performing multiple clustering runs on randomly sampled patristic distance distributions (1 million for each run) in Phylopart v2 (46).

\begin{tabular}{|c|c|c|c|c|c|}
\hline clustername & bootstrap & leafname & branchPath & medianOfDistances & sequencesperCluster \\
\hline 1380 & 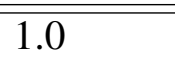 & "EPI_ISL_428205 & 6.69589999999999E-4 & 7.450999999999985E-5 & 2 \\
\hline 1436 & 1.0 & EPI_ISL_421391 & 6.329599999999989E-4 & 4.098999999999986E-5 & 4 \\
\hline 1436 & 1.0 & EPI_ISL_428385 & 6.329599999999989E-4 & 4.098999999999986E-5 & 4 \\
\hline 1436 & 1.0 & EPI_ISL_421623 & 6.65229999999999E-4 & 4.098999999999986E-5 & 4 \\
\hline 1436 & 1.0 & EPI_ISL_428394 & 6.28599999999999E-4 & 4.098999999999986E-5 & 4 \\
\hline 1443 & 1.0 & EPI_ISL_429588 & 6.627299999999991E-4 & 7.318999999999989E-5 & 2 \\
\hline 1443 & 1.0 & EPI_ISL_414625 & 6.62719999999999E-4 & 7.318999999999989E-5 & 2 \\
\hline 1483 & 1.0 & EPI_ISL_427742 & 6.758099999999988E-4 & $3.877000000000004 \mathrm{E}-5$ & 2 \\
\hline 1483 & 1.0 & EPI_ISL_421636 & 7.102199999999988E-4 & $3.877000000000004 \mathrm{E}-5$ & 2 \\
\hline 1507 & 1.0 & EPI_ISL_426837 & 7.282799999999986E-4 & 4.317999999999987E-5 & 10 \\
\hline 1507 & 1.0 & EPI_ISL_419943 & 7.369999999999984E-4 & 4.317999999999987E-5 & 10 \\
\hline 1507 & 1.0 & EPI_ISL_426860 & 7.714599999999984E-4 & 4.317999999999987E-5 & 10 \\
\hline 1507 & 1.0 & EPI_ISL_426729 & 7.326399999999985E-4 & 4.317999999999987E-5 & 10 \\
\hline 1507 & 1.0 & EPI_ISL_427764 & 7.391799999999984E-4 & 4.317999999999987E-5 & 10 \\
\hline 1507 & 1.0 & EPI_ISL_426862 & 7.714599999999984E-4 & 4.317999999999987E-5 & 10 \\
\hline 1507 & 1.0 & EPI_ISL_426725 & 7.304599999999985E-4 & 4.317999999999987E-5 & 10 \\
\hline 1507 & 1.0 & EPI_ISL_427682 & 7.391799999999984E-4 & 4.317999999999987E-5 & 10 \\
\hline 1507 & 1.0 & EPI_ISL_427775 & 7.765999999999984E-4 & 4.317999999999987E-5 & 10 \\
\hline 1507 & 1.0 & EPI_ISL_427683 & 7.765999999999984E-4 & 4.317999999999987E-5 & 10 \\
\hline 1531 & 1.0 & EPI_ISL_427095 & 6.976099999999984E-4 & $3.877000000000004 \mathrm{E}-5$ & 4 \\
\hline 1531 & 1.0 & EPI_ISL_419956 & 6.976099999999984E-4 & $3.877000000000004 \mathrm{E}-5$ & 4 \\
\hline 1531 & 1.0 & EPI_ISL_427094 & 7.320199999999984E-4 & $3.877000000000004 \mathrm{E}-5$ & 4 \\
\hline 1531 & 1.0 & EPI_ISL_427092 & 7.320199999999984E-4 & $3.877000000000004 \mathrm{E}-5$ & 4 \\
\hline 1601 & 1.0 & EPI_ISL_424170 & 6.890299999999993E-4 & 4.359999999999911E-6 & 2 \\
\hline 1601 & 1.0 & EPI_ISL_423020 & 6.890299999999993E-4 & 4.359999999999911E-6 & 2 \\
\hline 1605 & 1.0 & EPI_ISL_418204 & 6.518199999999994E-4 & $4.133000000000001 \mathrm{E}-5$ & 6 \\
\hline
\end{tabular}


Table S4: Clusters identified using a genetic distance threshold within $1 \%$ of the distribution of patristic distances within the entire tree. The minimum percentile threshold that maximized the number of clusters was chosen as the optimal threshold by performing multiple clustering runs on randomly sampled patristic distance distributions (1 million for each run) in Phylopart v2 (46).

\begin{tabular}{|c|c|c|c|c|c|}
\hline clustername & bootstrap & leafname & branchPath & medianOfDistances & sequencesperCluster \\
\hline 1605 & 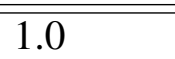 & "EPI_ISL_429023 & "7.548399999999995E-4 & $4.133000000000001 \mathrm{E}-5$ & 6 \\
\hline 1605 & 1.0 & EPI_ISL_417665 & 6.518199999999994E-4 & $4.133000000000001 \mathrm{E}-5$ & 6 \\
\hline 1605 & 1.0 & EPI_ISL_426951 & 6.862499999999994E-4 & $4.133000000000001 \mathrm{E}-5$ & 6 \\
\hline 1605 & 1.0 & EPI_ISL_430031 & 6.844299999999994E-4 & $4.133000000000001 \mathrm{E}-5$ & 6 \\
\hline 1605 & 1.0 & EPI_ISL_418192 & 6.518199999999994E-4 & $4.133000000000001 \mathrm{E}-5$ & 6 \\
\hline 1623 & 1.0 & EPI_ISL_430053 & $6.627499999999991 \mathrm{E}-4$ & $1.5259999999999796 \mathrm{E}-5$ & 8 \\
\hline 1623 & 1.0 & EPI_ISL_430062 & 7.368799999999991E-4 & $1.5259999999999796 \mathrm{E}-5$ & 8 \\
\hline 1623 & 1.0 & EPI_ISL_430056 & 6.562099999999992E-4 & $1.5259999999999796 \mathrm{E}-5$ & 8 \\
\hline 1623 & 1.0 & EPI_ISL_420812 & 6.64929999999999E-4 & $1.5259999999999796 \mathrm{E}-5$ & 8 \\
\hline 1623 & 1.0 & EPI_ISL_430061 & 6.64929999999999E-4 & $1.5259999999999796 \mathrm{E}-5$ & 8 \\
\hline 1623 & 1.0 & EPI_ISL_417977 & 6.950999999999992E-4 & $1.5259999999999796 \mathrm{E}-5$ & 8 \\
\hline 1623 & 1.0 & EPI_ISL_430054 & $6.627499999999991 \mathrm{E}-4$ & $1.5259999999999796 \mathrm{E}-5$ & 8 \\
\hline 1623 & 1.0 & EPI_ISL_430055 & $6.605699999999991 \mathrm{E}-4$ & $1.5259999999999796 \mathrm{E}-5$ & 8 \\
\hline 1640 & 0.988 & EPI_ISL_426748 & $6.172499999999992 \mathrm{E}-4$ & 6.539999999999758E-6 & 4 \\
\hline 1640 & 0.988 & EPI_ISL_419952 & $6.194299999999992 \mathrm{E}-4$ & 6.539999999999758E-6 & 4 \\
\hline 1640 & 0.988 & EPI_ISL_419954 & $6.194299999999992 \mathrm{E}-4$ & 6.539999999999758E-6 & 4 \\
\hline 1640 & 0.988 & EPI_ISL_419880 & $6.194299999999992 \mathrm{E}-4$ & 6.539999999999758E-6 & 4 \\
\hline 1666 & 1.0 & EPI_ISL_420759 & $6.633899999999991 \mathrm{E}-4$ & 8.686999999999974E-5 & 49 \\
\hline 1666 & 1.0 & EPI_ISL_424161 & $6.457499999999986 \mathrm{E}-4$ & 8.686999999999974E-5 & 49 \\
\hline 1666 & 1.0 & EPI_ISL_418076 & $6.217699999999991 \mathrm{E}-4$ & 8.686999999999974E-5 & 49 \\
\hline 1666 & 1.0 & EPI_ISL_429128 & 6.824799999999994E-4 & 8.686999999999974E-5 & 49 \\
\hline 1666 & 1.0 & EPI_ISL_426746 & $6.629499999999991 \mathrm{E}-4$ & 8.686999999999974E-5 & 49 \\
\hline 1666 & 1.0 & EPI_ISL_420704 & $7.529299999999988 \mathrm{E}-4$ & 8.686999999999974E-5 & 49 \\
\hline 1666 & 1.0 & EPI_ISL_418690 & $6.217699999999991 \mathrm{E}-4$ & 8.686999999999974E-5 & 49 \\
\hline 1666 & 1.0 & EPI_ISL_422035 & $6.627299999999991 \mathrm{E}-4$ & 8.686999999999974E-5 & 49 \\
\hline 1666 & 1.0 & EPI_ISL_425525 & 6.217699999999991E-4 & 8.686999999999974E-5 & 49 \\
\hline
\end{tabular}


Table S4: Clusters identified using a genetic distance threshold within $1 \%$ of the distribution of patristic distances within the entire tree. The minimum percentile threshold that maximized the number of clusters was chosen as the optimal threshold by performing multiple clustering runs on randomly sampled patristic distance distributions (1 million for each run) in Phylopart v2 (46).

\begin{tabular}{|c|c|c|c|c|c|}
\hline clustername & bootstrap & leafname & branchPath & medianOfDistances & sequencesperCluster \\
\hline 1666 & 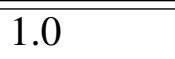 & "EPI_ISL_420695 & 6.348499999999989E-4 & 8.686999999999974E-5 & 49 \\
\hline 1666 & 1.0 & EPI_ISL_429161 & 6.217699999999991E-4 & 8.686999999999974E-5 & 49 \\
\hline 1666 & 1.0 & EPI_ISL_423608 & 6.435699999999987E-4 & 8.686999999999974E-5 & 49 \\
\hline 1666 & 1.0 & EPI_ISL_425519 & 6.217699999999991E-4 & 8.686999999999974E-5 & 49 \\
\hline 1666 & 1.0 & EPI_ISL_423596 & 6.778099999999989E-4 & 8.686999999999974E-5 & 49 \\
\hline 1666 & 1.0 & EPI_ISL_422696 & 6.217699999999991E-4 & 8.686999999999974E-5 & 49 \\
\hline 1666 & 1.0 & EPI_ISL_429127 & 6.561799999999992E-4 & 8.686999999999974E-5 & 49 \\
\hline 1666 & 1.0 & EPI_ISL_422081 & 7.613999999999992E-4 & 8.686999999999974E-5 & 49 \\
\hline 1666 & 1.0 & EPI_ISL_423609 & 6.33229999999999E-4 & 8.686999999999974E-5 & 49 \\
\hline 1666 & 1.0 & EPI_ISL_421005 & 7.452199999999988E-4 & 8.686999999999974E-5 & 49 \\
\hline 1666 & 1.0 & EPI_ISL_420459 & 6.174099999999992E-4 & 8.686999999999974E-5 & 49 \\
\hline 1666 & 1.0 & EPI_ISL_425388 & 7.600699999999985E-4 & 8.686999999999974E-5 & 49 \\
\hline 1666 & 1.0 & EPI_ISL_423655 & 6.587699999999987E-4 & 8.686999999999974E-5 & 49 \\
\hline 1666 & 1.0 & EPI_ISL_424598 & 6.217699999999991E-4 & 8.686999999999974E-5 & 49 \\
\hline 1666 & 1.0 & EPI_ISL_419428 & $6.217699999999991 \mathrm{E}-4$ & 8.686999999999974E-5 & 49 \\
\hline 1666 & 1.0 & EPI_ISL_423086 & 6.539999999999992E-4 & 8.686999999999974E-5 & 49 \\
\hline 1666 & 1.0 & EPI_ISL_422124 & 7.75279999999999E-4 & 8.686999999999974E-5 & 49 \\
\hline 1666 & 1.0 & EPI_ISL_422777 & 6.670799999999989E-4 & 8.686999999999974E-5 & 49 \\
\hline 1666 & 1.0 & EPI_ISL_423768 & 6.670799999999989E-4 & 8.686999999999974E-5 & 49 \\
\hline 1666 & 1.0 & EPI_ISL_423386 & 6.26129999999999E-4 & 8.686999999999974E-5 & 49 \\
\hline 1666 & 1.0 & EPI_ISL_422211 & 7.430399999999988E-4 & 8.686999999999974E-5 & 49 \\
\hline 1666 & 1.0 & EPI_ISL_422656 & 7.444199999999989E-4 & 8.686999999999974E-5 & 49 \\
\hline 1666 & 1.0 & EPI_ISL_422164 & 7.269899999999992E-4 & 8.686999999999974E-5 & 49 \\
\hline 1666 & 1.0 & EPI_ISL_429126 & 6.217699999999991E-4 & 8.686999999999974E-5 & 49 \\
\hline 1666 & 1.0 & EPI_ISL_429162 & $6.217699999999991 \mathrm{E}-4$ & 8.686999999999974E-5 & 49 \\
\hline 1666 & 1.0 & EPI_ISL_424402 & $6.217699999999991 \mathrm{E}-4$ & 8.686999999999974E-5 & 49 \\
\hline
\end{tabular}


Table S4: Clusters identified using a genetic distance threshold within $1 \%$ of the distribution of patristic distances within the entire tree. The minimum percentile threshold that maximized the number of clusters was chosen as the optimal threshold by performing multiple clustering runs on randomly sampled patristic distance distributions (1 million for each run) in Phylopart v2 (46).

\begin{tabular}{|c|c|c|c|c|c|}
\hline clustername & bootstrap & leafname & branchPath & medianOfDistances & sequencesperCluster \\
\hline 1666 & 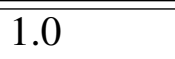 & "EPI_ISL_422655 & 7.444199999999989E-4 & 8.686999999999974E-5 & 49 \\
\hline 1666 & 1.0 & EPI_ISL_422959 & 6.130499999999993E-4 & 8.686999999999974E-5 & 49 \\
\hline 1666 & 1.0 & EPI_ISL_421853 & 6.195899999999992E-4 & 8.686999999999974E-5 & 49 \\
\hline 1666 & 1.0 & EPI_ISL_428148 & 7.12689999999999E-4 & 8.686999999999974E-5 & 49 \\
\hline 1666 & 1.0 & EPI_ISL_420752 & 6.802299999999987E-4 & 8.686999999999974E-5 & 49 \\
\hline 1666 & 1.0 & EPI_ISL_423220 & 6.26129999999999E-4 & 8.686999999999974E-5 & 49 \\
\hline 1666 & 1.0 & EPI_ISL_423563 & 6.37039999999999E-4 & 8.686999999999974E-5 & 49 \\
\hline 1666 & 1.0 & EPI_ISL_429558 & 6.736199999999988E-4 & 8.686999999999974E-5 & 49 \\
\hline 1666 & 1.0 & EPI_ISL_420933 & 6.842399999999986E-4 & 8.686999999999974E-5 & 49 \\
\hline 1666 & 1.0 & EPI_ISL_422042 & 7.452199999999988E-4 & 8.686999999999974E-5 & 49 \\
\hline 1666 & 1.0 & EPI_ISL_424585 & 6.217699999999991E-4 & 8.686999999999974E-5 & 49 \\
\hline 1666 & 1.0 & EPI_ISL_420221 & 7.185999999999986E-4 & 8.686999999999974E-5 & 49 \\
\hline 1666 & 1.0 & EPI_ISL_423932 & 7.151999999999987E-4 & 8.686999999999974E-5 & 49 \\
\hline 1666 & 1.0 & EPI_ISL_423317 & 6.217699999999991E-4 & 8.686999999999974E-5 & 49 \\
\hline 1771 & 1.0 & EPI_ISL_418184 & 7.875099999999995E-4 & 3.877999999999998E-5 & 3 \\
\hline 1771 & 1.0 & EPI_ISL_427446 & 7.530899999999994E-4 & 3.877999999999998E-5 & 3 \\
\hline 1771 & 1.0 & EPI_ISL_427462 & 7.530899999999994E-4 & 3.877999999999998E-5 & 3 \\
\hline 1782 & 1.0 & EPI_ISL_426799 & 6.759199999999996E-4 & 4.312999999999995E-5 & 5 \\
\hline 1782 & 1.0 & EPI_ISL_426809 & 7.125699999999996E-4 & 4.312999999999995E-5 & 5 \\
\hline 1782 & 1.0 & EPI_ISL_427006 & 7.083899999999996E-4 & 4.312999999999995E-5 & 5 \\
\hline 1782 & 1.0 & EPI_ISL_427160 & 6.802799999999995E-4 & 4.312999999999995E-5 & 5 \\
\hline 1782 & 1.0 & EPI_ISL_426940 & 7.146899999999995E-4 & 4.312999999999995E-5 & 5 \\
\hline 1794 & 1.0 & EPI_ISL_417516 & 7.125199999999996E-4 & 7.597000000000007E-5 & 2 \\
\hline 1794 & 1.0 & EPI_ISL_423971 & 6.409099999999995E-4 & 7.597000000000007E-5 & 2 \\
\hline 1816 & 1.0 & EPI_ISL_417752 & 7.471099999999989E-4 & 6.928999999999941E-5 & 48 \\
\hline 1816 & 1.0 & EPI_ISL_417806 & 6.602399999999991E-4 & 6.928999999999941E-5 & 48 \\
\hline
\end{tabular}


Table S4: Clusters identified using a genetic distance threshold within $1 \%$ of the distribution of patristic distances within the entire tree. The minimum percentile threshold that maximized the number of clusters was chosen as the optimal threshold by performing multiple clustering runs on randomly sampled patristic distance distributions (1 million for each run) in Phylopart v2 (46).

\begin{tabular}{|c|c|c|c|c|c|}
\hline clustername & bootstrap & leafname & branchPath & medianOfDistances & sequencesperCluster \\
\hline 1816 & 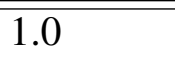 & "EPI_ISL_422914 & 7.492999999999988E-4 & $6.928999999999941 \mathrm{E}-5$ & 48 \\
\hline 1816 & 1.0 & EPI_ISL_425409 & 6.99009999999999E-4 & 6.928999999999941E-5 & 48 \\
\hline 1816 & 1.0 & EPI_ISL_421501 & 6.602399999999991E-4 & 6.928999999999941E-5 & 48 \\
\hline 1816 & 1.0 & EPI_ISL_427721 & 7.531499999999988E-4 & 6.928999999999941E-5 & 48 \\
\hline 1816 & 1.0 & EPI_ISL_429729 & 7.39969999999999E-4 & 6.928999999999941E-5 & 48 \\
\hline 1816 & 1.0 & EPI_ISL_423443 & 7.099099999999988E-4 & 6.928999999999941E-5 & 48 \\
\hline 1816 & 1.0 & EPI_ISL_423797 & 7.120899999999988E-4 & 6.928999999999941E-5 & 48 \\
\hline 1816 & 1.0 & EPI_ISL_418206 & 6.711399999999989E-4 & 6.928999999999941E-5 & 48 \\
\hline 1816 & 1.0 & EPI_ISL_422568 & 6.602399999999991E-4 & 6.928999999999941E-5 & 48 \\
\hline 1816 & 1.0 & EPI_ISL_422954 & 7.041399999999989E-4 & 6.928999999999941E-5 & 48 \\
\hline 1816 & 1.0 & EPI_ISL_422858 & 7.041399999999989E-4 & 6.928999999999941E-5 & 48 \\
\hline 1816 & 1.0 & EPI_ISL_422856 & 7.01099999999999E-4 & 6.928999999999941E-5 & 48 \\
\hline 1816 & 1.0 & EPI_ISL_423445 & 7.319499999999991E-4 & 6.928999999999941E-5 & 48 \\
\hline 1816 & 1.0 & EPI_ISL_423163 & 6.602399999999991E-4 & 6.928999999999941E-5 & 48 \\
\hline 1816 & 1.0 & EPI_ISL_422620 & 7.874499999999988E-4 & 6.928999999999941E-5 & 48 \\
\hline 1816 & 1.0 & EPI_ISL_423643 & 6.689599999999989E-4 & 6.928999999999941E-5 & 48 \\
\hline 1816 & 1.0 & EPI_ISL_420791 & 6.602399999999991E-4 & 6.928999999999941E-5 & 48 \\
\hline 1816 & 1.0 & EPI_ISL_424042 & 7.055499999999989E-4 & 6.928999999999941E-5 & 48 \\
\hline 1816 & 1.0 & EPI_ISL_425322 & 6.99009999999999E-4 & 6.928999999999941E-5 & 48 \\
\hline 1816 & 1.0 & EPI_ISL_418207 & 6.602399999999991E-4 & 6.928999999999941E-5 & 48 \\
\hline 1816 & 1.0 & EPI_ISL_422658 & 6.624199999999991E-4 & 6.928999999999941E-5 & 48 \\
\hline 1816 & 1.0 & EPI_ISL_423603 & 7.03369999999999E-4 & 6.928999999999941E-5 & 48 \\
\hline 1816 & 1.0 & EPI_ISL_421560 & 6.711399999999989E-4 & 6.928999999999941E-5 & 48 \\
\hline 1816 & 1.0 & EPI_ISL_420048 & 7.449299999999989E-4 & 6.928999999999941E-5 & 48 \\
\hline 1816 & 1.0 & EPI_ISL_420058 & 6.602399999999991E-4 & 6.928999999999941E-5 & 48 \\
\hline 1816 & 1.0 & EPI_ISL_427136 & 6.946599999999992E-4 & 6.928999999999941E-5 & 48 \\
\hline
\end{tabular}


Table S4: Clusters identified using a genetic distance threshold within $1 \%$ of the distribution of patristic distances within the entire tree. The minimum percentile threshold that maximized the number of clusters was chosen as the optimal threshold by performing multiple clustering runs on randomly sampled patristic distance distributions (1 million for each run) in Phylopart v2 (46).

\begin{tabular}{|c|c|c|c|c|c|}
\hline clustername & bootstrap & leafname & branchPath & medianOfDistances & sequencesperCluster \\
\hline 1816 & 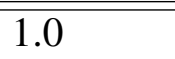 & "EPI_ISL_422903 & 7.041399999999989E-4 & 6.928999999999941E-5 & $\overline{48}$ \\
\hline 1816 & 1.0 & EPI_ISL_418209 & 6.924699999999992E-4 & 6.928999999999941E-5 & 48 \\
\hline 1816 & 1.0 & EPI_ISL_422570 & 7.351199999999992E-4 & 6.928999999999941E-5 & 48 \\
\hline 1816 & 1.0 & EPI_ISL_420062 & 7.313399999999992E-4 & 6.928999999999941E-5 & 48 \\
\hline 1816 & 1.0 & EPI_ISL_423861 & 7.077299999999989E-4 & 6.928999999999941E-5 & 48 \\
\hline 1816 & 1.0 & EPI_ISL_423332 & 7.03369999999999E-4 & 6.928999999999941E-5 & 48 \\
\hline 1816 & 1.0 & EPI_ISL_425694 & 6.711399999999989E-4 & 6.928999999999941E-5 & 48 \\
\hline 1816 & 1.0 & EPI_ISL_429737 & 7.05559999999999E-4 & 6.928999999999941E-5 & 48 \\
\hline 1816 & 1.0 & EPI_ISL_423411 & 7.120899999999988E-4 & 6.928999999999941E-5 & 48 \\
\hline 1816 & 1.0 & EPI_ISL_423564 & 7.74479999999999E-4 & 6.928999999999941E-5 & 48 \\
\hline 1816 & 1.0 & EPI_ISL_420792 & 6.602399999999991E-4 & 6.928999999999941E-5 & 48 \\
\hline 1816 & 1.0 & EPI_ISL_424877 & 6.602399999999991E-4 & 6.928999999999941E-5 & 48 \\
\hline 1816 & 1.0 & EPI_ISL_422575 & 6.602399999999991E-4 & 6.928999999999941E-5 & 48 \\
\hline 1816 & 1.0 & EPI_ISL_418208 & 6.580599999999992E-4 & 6.928999999999941E-5 & 48 \\
\hline 1816 & 1.0 & EPI_ISL_418420 & 6.926199999999992E-4 & 6.928999999999941E-5 & 48 \\
\hline 1816 & 1.0 & EPI_ISL_423796 & 7.120899999999988E-4 & 6.928999999999941E-5 & 48 \\
\hline 1816 & 1.0 & EPI_ISL_423862 & 6.946499999999991E-4 & 6.928999999999941E-5 & 48 \\
\hline 1816 & 1.0 & EPI_ISL_422788 & 6.602399999999991E-4 & 6.928999999999941E-5 & 48 \\
\hline 1816 & 1.0 & EPI_ISL_417629 & 6.602399999999991E-4 & 6.928999999999941E-5 & 48 \\
\hline 1816 & 1.0 & EPI_ISL_422566 & 6.602399999999991E-4 & 6.928999999999941E-5 & 48 \\
\hline 1942 & 1.0 & EPI_ISL_427151 & 6.065099999999994E-4 & 4.359999999999911E-6 & 2 \\
\hline 1942 & 1.0 & EPI_ISL_427802 & 6.065099999999994E-4 & 4.359999999999911E-6 & 2 \\
\hline 1974 & 1.0 & EPI_ISL_426562 & 7.167099999999987E-4 & 0.0 & 6 \\
\hline 1974 & 1.0 & EPI_ISL_426563 & 7.167099999999987E-4 & 0.0 & 6 \\
\hline 1974 & 1.0 & EPI_ISL_426565 & 7.167099999999987E-4 & 0.0 & 6 \\
\hline 1974 & 1.0 & EPI_ISL_426567 & 7.167099999999987E-4 & 0.0 & 6 \\
\hline
\end{tabular}


Table S4: Clusters identified using a genetic distance threshold within $1 \%$ of the distribution of patristic distances within the entire tree. The minimum percentile threshold that maximized the number of clusters was chosen as the optimal threshold by performing multiple clustering runs on randomly sampled patristic distance distributions (1 million for each run) in Phylopart v2 (46).

\begin{tabular}{|c|c|c|c|c|c|}
\hline clustername & bootstrap & leafname & branchPath & medianOfDistances & sequencesperCluster \\
\hline 1974 & 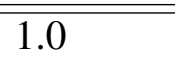 & "EPI_ISL_426561 & 7.167099999999987E-4 & 0.0 & $\overline{6} 6$ \\
\hline 1974 & 1.0 & EPI_ISL_426566 & 8.610599999999987E-4 & 0.0 & 6 \\
\hline 1997 & 1.0 & EPI_ISL_422461 & 7.510999999999987E-4 & 7.811999999999988E-5 & 5 \\
\hline 1997 & 1.0 & EPI_ISL_426161 & $9.322499999999987 \mathrm{E}-4$ & 7.811999999999988E-5 & 5 \\
\hline 1997 & 1.0 & EPI_ISL_429847 & 8.248599999999987E-4 & 7.811999999999988E-5 & 5 \\
\hline 1997 & 1.0 & EPI_ISL_429846 & 8.248599999999987E-4 & 7.811999999999988E-5 & 5 \\
\hline 1997 & 1.0 & EPI_ISL_429848 & 8.248599999999987E-4 & 7.811999999999988E-5 & 5 \\
\hline 2026 & 1.0 & EPI_ISL_419778 & 6.627099999999991E-4 & 4.359999999999911E-6 & 2 \\
\hline 2026 & 1.0 & EPI_ISL_419742 & 6.627099999999991E-4 & 4.359999999999911E-6 & 2 \\
\hline 2059 & 1.0 & EPI_ISL_416478 & 6.539999999999993E-4 & 7.317999999999995E-5 & 2 \\
\hline 2059 & 1.0 & EPI_ISL_424591 & 6.539999999999993E-4 & 7.317999999999995E-5 & 2 \\
\hline 2071 & 1.0 & EPI_ISL_421394 & 6.130599999999994E-4 & 4.359999999999911E-6 & 2 \\
\hline 2071 & 1.0 & EPI_ISL_421412 & 6.130599999999994E-4 & 4.359999999999911E-6 & 2 \\
\hline 2098 & 1.0 & EPI_ISL_422086 & 7.704899999999991E-4 & 3.877999999999998E-5 & 2 \\
\hline 2098 & 1.0 & EPI_ISL_420745 & 7.36069999999999E-4 & 3.877999999999998E-5 & 2 \\
\hline 2103 & 1.0 & EPI_ISL_419188 & 6.26129999999999E-4 & 7.597000000000007E-5 & 2 \\
\hline 2103 & 1.0 & EPI_ISL_422932 & 6.977399999999991E-4 & 7.597000000000007E-5 & 2 \\
\hline 2112 & 1.0 & EPI_ISL_428951 & 6.976899999999992E-4 & 8.023999999999987E-5 & 2 \\
\hline 2112 & 1.0 & EPI_ISL_419708 & $6.218099999999991 \mathrm{E}-4$ & 8.023999999999987E-5 & 2 \\
\hline 2125 & 1.0 & EPI_ISL_417579 & 6.67279999999999E-4 & 4.178999999999988E-5 & 3 \\
\hline 2125 & 1.0 & EPI_ISL_424388 & 6.67279999999999E-4 & 4.178999999999988E-5 & 3 \\
\hline 2125 & 1.0 & EPI_ISL_424501 & $7.003499999999991 \mathrm{E}-4$ & 4.178999999999988E-5 & 3 \\
\hline 2132 & 1.0 & EPI_ISL_426744 & $6.217599999999992 \mathrm{E}-4$ & $3.8979999999999874 \mathrm{E}-5$ & 2 \\
\hline 2132 & 1.0 & EPI_ISL_422532 & 6.563799999999993E-4 & $3.8979999999999874 \mathrm{E}-5$ & 2 \\
\hline 2151 & 1.0 & EPI_ISL_423168 & 6.671299999999991E-4 & 4.359999999999911E-6 & 2 \\
\hline 2151 & 1.0 & EPI_ISL_422008 & 6.671299999999991E-4 & 4.359999999999911E-6 & 2 \\
\hline
\end{tabular}


Table S4: Clusters identified using a genetic distance threshold within $1 \%$ of the distribution of patristic distances within the entire tree. The minimum percentile threshold that maximized the number of clusters was chosen as the optimal threshold by performing multiple clustering runs on randomly sampled patristic distance distributions (1 million for each run) in Phylopart v2 (46).

\begin{tabular}{|c|c|c|c|c|c|}
\hline clustername & bootstrap & leafname & branchPath & medianOfDistances & sequencesperCluster \\
\hline 2159 & 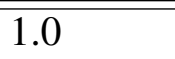 & "EPI_ISL_426994 & 6.65149999999999E-4 & 5.697999999999988E-5 & $\overline{5}$ \\
\hline 2159 & 1.0 & EPI_ISL_426972 & 6.627299999999991E-4 & 5.697999999999988E-5 & 5 \\
\hline 2159 & 1.0 & EPI_ISL_426866 & 6.26149999999999E-4 & 5.697999999999988E-5 & 5 \\
\hline 2159 & 1.0 & EPI_ISL_426987 & 6.80609999999999E-4 & 5.697999999999988E-5 & 5 \\
\hline 2159 & 1.0 & EPI_ISL_426872 & 6.30509999999999E-4 & 5.697999999999988E-5 & 5 \\
\hline 2174 & 1.0 & EPI_ISL_429572 & 6.949599999999984E-4 & 8.017999999999979E-5 & 25 \\
\hline 2174 & 1.0 & EPI_ISL_429530 & 7.40409999999999E-4 & 8.017999999999979E-5 & 25 \\
\hline 2174 & 1.0 & EPI_ISL_429312 & 7.184699999999986E-4 & 8.017999999999979E-5 & 25 \\
\hline 2174 & 1.0 & EPI_ISL_429478 & 7.228199999999986E-4 & 8.017999999999979E-5 & 25 \\
\hline 2174 & 1.0 & EPI_ISL_429468 & 6.949599999999984E-4 & 8.017999999999979E-5 & 25 \\
\hline 2174 & 1.0 & EPI_ISL_429332 & $6.753399999999988 \mathrm{E}-4$ & 8.017999999999979E-5 & 25 \\
\hline 2174 & 1.0 & EPI_ISL_429565 & 7.075599999999989E-4 & 8.017999999999979E-5 & 25 \\
\hline 2174 & 1.0 & EPI_ISL_429529 & 6.949599999999984E-4 & 8.017999999999979E-5 & 25 \\
\hline 2174 & 1.0 & EPI_ISL_426795 & 6.66619999999999E-4 & 8.017999999999979E-5 & 25 \\
\hline 2174 & 1.0 & EPI_ISL_429532 & 7.831099999999989E-4 & 8.017999999999979E-5 & 25 \\
\hline 2174 & 1.0 & EPI_ISL_429327 & 6.949599999999984E-4 & 8.017999999999979E-5 & 25 \\
\hline 2174 & 1.0 & EPI_ISL_429311 & 7.075599999999989E-4 & 8.017999999999979E-5 & 25 \\
\hline 2174 & 1.0 & EPI_ISL_429589 & 7.119199999999988E-4 & 8.017999999999979E-5 & 25 \\
\hline 2174 & 1.0 & EPI_ISL_429570 & 7.271799999999985E-4 & 8.017999999999979E-5 & 25 \\
\hline 2174 & 1.0 & EPI_ISL_429465 & 6.949599999999984E-4 & 8.017999999999979E-5 & 25 \\
\hline 2174 & 1.0 & EPI_ISL_429541 & 7.838699999999988E-4 & 8.017999999999979E-5 & 25 \\
\hline 2174 & 1.0 & EPI_ISL_429302 & 6.949599999999984E-4 & 8.017999999999979E-5 & 25 \\
\hline 2174 & 1.0 & EPI_ISL_429461 & 7.621999999999985E-4 & 8.017999999999979E-5 & 25 \\
\hline 2174 & 1.0 & EPI_ISL_429578 & 6.949599999999984E-4 & 8.017999999999979E-5 & 25 \\
\hline 2174 & 1.0 & EPI_ISL_429535 & 7.228199999999986E-4 & 8.017999999999979E-5 & 25 \\
\hline 2174 & 1.0 & EPI_ISL_429508 & 7.40409999999999E-4 & 8.017999999999979E-5 & 25 \\
\hline
\end{tabular}


Table S4: Clusters identified using a genetic distance threshold within $1 \%$ of the distribution of patristic distances within the entire tree. The minimum percentile threshold that maximized the number of clusters was chosen as the optimal threshold by performing multiple clustering runs on randomly sampled patristic distance distributions (1 million for each run) in Phylopart v2 (46).

\begin{tabular}{|c|c|c|c|c|c|}
\hline clustername & bootstrap & leafname & branchPath & medianOfDistances & sequencesperCluster \\
\hline 2174 & 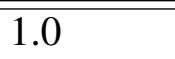 & "EPI_ISL_429566 & 7.140999999999988E-4 & 8.017999999999979E-5 & 25 \\
\hline 2174 & 1.0 & EPI_ISL_429310 & 7.959099999999985E-4 & 8.017999999999979E-5 & 25 \\
\hline 2174 & 1.0 & EPI_ISL_429294 & 7.167599999999987E-4 & 8.017999999999979E-5 & 25 \\
\hline 2174 & 1.0 & EPI_ISL_429300 & 6.949599999999984E-4 & 8.017999999999979E-5 & 25 \\
\hline 2229 & 1.0 & EPI_ISL_421382 & 6.326699999999989E-4 & 4.0949999999999884E-5 & 4 \\
\hline 2229 & 1.0 & EPI_ISL_429492 & 6.64899999999999E-4 & 4.0949999999999884E-5 & 4 \\
\hline 2229 & 1.0 & EPI_ISL_421419 & 6.348499999999989E-4 & 4.0949999999999884E-5 & 4 \\
\hline 2229 & 1.0 & EPI_ISL_422538 & 6.348499999999989E-4 & 4.0949999999999884E-5 & 4 \\
\hline 2260 & 1.0 & EPI_ISL_418652 & 7.579799999999987E-4 & 8.407999999999962E-5 & 12 \\
\hline 2260 & 1.0 & EPI_ISL_418638 & 7.901899999999988E-4 & 8.407999999999962E-5 & 12 \\
\hline 2260 & 1.0 & EPI_ISL_418798 & 7.254499999999986E-4 & 8.407999999999962E-5 & 12 \\
\hline 2260 & 1.0 & EPI_ISL_418644 & 7.610599999999986E-4 & 8.407999999999962E-5 & 12 \\
\hline 2260 & 1.0 & EPI_ISL_420442 & 7.598599999999986E-4 & 8.407999999999962E-5 & 12 \\
\hline 2260 & 1.0 & EPI_ISL_427347 & 7.892799999999988E-4 & 8.407999999999962E-5 & 12 \\
\hline 2260 & 1.0 & EPI_ISL_421184 & 7.920899999999987E-4 & 8.407999999999962E-5 & 12 \\
\hline 2260 & 1.0 & EPI_ISL_417006 & 7.210899999999987E-4 & 8.407999999999962E-5 & 12 \\
\hline 2260 & 1.0 & EPI_ISL_424639 & 7.210899999999987E-4 & 8.407999999999962E-5 & 12 \\
\hline 2260 & 1.0 & EPI_ISL_427390 & 7.920899999999987E-4 & 8.407999999999962E-5 & 12 \\
\hline 2260 & 1.0 & EPI_ISL_427369 & $8.711599999999986 \mathrm{E}-4$ & 8.407999999999962E-5 & 12 \\
\hline 2260 & 1.0 & EPI_ISL_424628 & 7.232699999999986E-4 & 8.407999999999962E-5 & 12 \\
\hline 2287 & 1.0 & EPI_ISL_429748 & 7.927999999999987E-4 & 7.35899999999999E-5 & 2 \\
\hline 2287 & 1.0 & EPI_ISL_429791 & 7.930299999999987E-4 & 7.35899999999999E-5 & 2 \\
\hline 2291 & 1.0 & EPI_ISL_429790 & 7.167299999999988E-4 & $3.8920000000000014 \mathrm{E}-5$ & 3 \\
\hline 2291 & 1.0 & EPI_ISL_429756 & 7.534699999999987E-4 & $3.8920000000000014 \mathrm{E}-5$ & 3 \\
\hline 2291 & 1.0 & EPI_ISL_429767 & 7.189099999999987E-4 & $3.8920000000000014 \mathrm{E}-5$ & 3 \\
\hline 2337 & 0.987 & EPI_ISL_430036 & 6.063499999999995E-4 & 4.0939999999999943E-5 & 7 \\
\hline
\end{tabular}


Table S4: Clusters identified using a genetic distance threshold within $1 \%$ of the distribution of patristic distances within the entire tree. The minimum percentile threshold that maximized the number of clusters was chosen as the optimal threshold by performing multiple clustering runs on randomly sampled patristic distance distributions (1 million for each run) in Phylopart v2 (46).

\begin{tabular}{|c|c|c|c|c|c|}
\hline clustername & bootstrap & leafname & branchPath & medianOfDistances & sequencesperCluster \\
\hline 2337 & 0.987 & EPI_ISL_430023 & 6.019899999999996E-4 & "4.0939999999999943E-5 & 7 \\
\hline 2337 & 0.987 & EPI_ISL_430029 & 6.041699999999996E-4 & 4.0939999999999943E-5 & 7 \\
\hline 2337 & 0.987 & EPI_ISL_430027 & 6.085299999999995E-4 & 4.0939999999999943E-5 & 7 \\
\hline 2337 & 0.987 & EPI_ISL_430034 & $6.334399999999998 \mathrm{E}-4$ & 4.0939999999999943E-5 & 7 \\
\hline 2337 & 0.987 & EPI_ISL_429121 & 6.342099999999997E-4 & 4.0939999999999943E-5 & 7 \\
\hline 2337 & 0.987 & EPI_ISL_430020 & 6.085299999999995E-4 & 4.0939999999999943E-5 & 7 \\
\hline 2369 & 1.0 & EPI_ISL_420575 & 6.731199999999997E-4 & 7.969999999999982E-5 & 13 \\
\hline 2369 & 1.0 & EPI_ISL_427162 & 7.081399999999997E-4 & 7.969999999999982E-5 & 13 \\
\hline 2369 & 1.0 & EPI_ISL_426053 & 7.190399999999995E-4 & 7.969999999999982E-5 & 13 \\
\hline 2369 & 1.0 & EPI_ISL_426839 & 7.485999999999997E-4 & 7.969999999999982E-5 & 13 \\
\hline 2369 & 1.0 & EPI_ISL_421578 & 6.517999999999994E-4 & 7.969999999999982E-5 & 13 \\
\hline 2369 & 1.0 & EPI_ISL_419703 & 6.774799999999996E-4 & 7.969999999999982E-5 & 13 \\
\hline 2369 & 1.0 & EPI_ISL_424304 & 6.796599999999996E-4 & 7.969999999999982E-5 & 13 \\
\hline 2369 & 1.0 & EPI_ISL_421363 & 6.840199999999995E-4 & 7.969999999999982E-5 & 13 \\
\hline 2369 & 1.0 & EPI_ISL_427562 & $6.539799999999993 \mathrm{E}-4$ & 7.969999999999982E-5 & 13 \\
\hline 2369 & 1.0 & EPI_ISL_427633 & $6.539799999999993 \mathrm{E}-4$ & 7.969999999999982E-5 & 13 \\
\hline 2369 & 1.0 & EPI_ISL_428388 & 6.696099999999997E-4 & 7.969999999999982E-5 & 13 \\
\hline 2369 & 1.0 & EPI_ISL_420296 & $6.731199999999997 \mathrm{E}-4$ & 7.969999999999982E-5 & 13 \\
\hline 2369 & 1.0 & EPI_ISL_421720 & 6.539799999999993E-4 & 7.969999999999982E-5 & 13 \\
\hline 2413 & 0.987 & EPI_ISL_426159 & 6.708799999999997E-4 & 3.875999999999988E-5 & 3 \\
\hline 2413 & 0.987 & EPI_ISL_422463 & 6.364799999999996E-4 & 3.875999999999988E-5 & 3 \\
\hline 2413 & 0.987 & EPI_ISL_419651 & 6.364799999999996E-4 & 3.875999999999988E-5 & 3 \\
\hline 2422 & 1.0 & EPI_ISL_416509 & 6.321799999999998E-4 & 4.0939999999999943E-5 & 3 \\
\hline 2422 & 1.0 & EPI_ISL_416512 & 5.999599999999996E-4 & 4.0939999999999943E-5 & 3 \\
\hline 2422 & 1.0 & EPI_ISL_416513 & 6.343599999999997E-4 & 4.0939999999999943E-5 & 3 \\
\hline 2434 & 1.0 & EPI_ISL_429639 & 6.847999999999994E-4 & 8.03599999999998E-5 & 22 \\
\hline
\end{tabular}


Table S4: Clusters identified using a genetic distance threshold within $1 \%$ of the distribution of patristic distances within the entire tree. The minimum percentile threshold that maximized the number of clusters was chosen as the optimal threshold by performing multiple clustering runs on randomly sampled patristic distance distributions (1 million for each run) in Phylopart v2 (46).

\begin{tabular}{|c|c|c|c|c|c|}
\hline clustername & bootstrap & leafname & branchPath & medianOfDistances & sequencesperCluster \\
\hline 2434 & 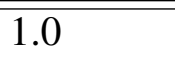 & "EPI_ISL_426118 & 6.30479999999999E-4 & "8.03599999999998E-5 & 22 \\
\hline 2434 & 1.0 & EPI_ISL_429644 & 7.117299999999996E-4 & 8.03599999999998E-5 & 22 \\
\hline 2434 & 1.0 & EPI_ISL_429605 & 6.388299999999996E-4 & 8.03599999999998E-5 & 22 \\
\hline 2434 & 1.0 & EPI_ISL_427198 & 6.626999999999991E-4 & 8.03599999999998E-5 & 22 \\
\hline 2434 & 1.0 & EPI_ISL_427248 & 6.282999999999991E-4 & 8.03599999999998E-5 & 22 \\
\hline 2434 & 1.0 & EPI_ISL_427235 & 6.173999999999993E-4 & 8.03599999999998E-5 & 22 \\
\hline 2434 & 1.0 & EPI_ISL_427211 & 6.282999999999991E-4 & 8.03599999999998E-5 & 22 \\
\hline 2434 & 1.0 & EPI_ISL_424172 & 6.933999999999992E-4 & 8.03599999999998E-5 & 22 \\
\hline 2434 & 1.0 & EPI_ISL_424293 & 6.282999999999991E-4 & 8.03599999999998E-5 & 22 \\
\hline 2434 & 1.0 & EPI_ISL_424237 & 6.282999999999991E-4 & 8.03599999999998E-5 & 22 \\
\hline 2434 & 1.0 & EPI_ISL_427268 & 6.715599999999996E-4 & 8.03599999999998E-5 & 22 \\
\hline 2434 & 1.0 & EPI_ISL_424299 & 7.188799999999994E-4 & 8.03599999999998E-5 & 22 \\
\hline 2434 & 1.0 & EPI_ISL_429633 & 6.336299999999997E-4 & 8.03599999999998E-5 & 22 \\
\hline 2434 & 1.0 & EPI_ISL_426105 & 6.086799999999995E-4 & 8.03599999999998E-5 & 22 \\
\hline 2434 & 1.0 & EPI_ISL_426090 & 6.408999999999996E-4 & 8.03599999999998E-5 & 22 \\
\hline 2434 & 1.0 & EPI_ISL_424300 & 6.282999999999991E-4 & 8.03599999999998E-5 & 22 \\
\hline 2434 & 1.0 & EPI_ISL_424325 & 6.583399999999992E-4 & 8.03599999999998E-5 & 22 \\
\hline 2434 & 1.0 & EPI_ISL_429629 & 7.461399999999996E-4 & 8.03599999999998E-5 & 22 \\
\hline 2434 & 1.0 & EPI_ISL_424187 & 6.195799999999992E-4 & 8.03599999999998E-5 & 22 \\
\hline 2434 & 1.0 & EPI_ISL_424290 & 6.152199999999993E-4 & 8.03599999999998E-5 & 22 \\
\hline 2434 & 1.0 & EPI_ISL_424291 & 6.30479999999999E-4 & 8.03599999999998E-5 & 22 \\
\hline 2483 & 1.0 & EPI_ISL_423021 & 6.649199999999997E-4 & 4.359999999999911E-6 & 4 \\
\hline 2483 & 1.0 & EPI_ISL_423019 & 6.649199999999997E-4 & 4.359999999999911E-6 & 4 \\
\hline 2483 & 1.0 & EPI_ISL_424234 & 6.649199999999997E-4 & 4.359999999999911E-6 & 4 \\
\hline 2483 & 1.0 & EPI_ISL_424239 & 6.649199999999997E-4 & 4.359999999999911E-6 & 4 \\
\hline 2495 & 1.0 & EPI_ISL_429494 & 6.498899999999994E-4 & 8.248999999999973E-5 & 50 \\
\hline
\end{tabular}


Table S4: Clusters identified using a genetic distance threshold within $1 \%$ of the distribution of patristic distances within the entire tree. The minimum percentile threshold that maximized the number of clusters was chosen as the optimal threshold by performing multiple clustering runs on randomly sampled patristic distance distributions (1 million for each run) in Phylopart v2 (46).

\begin{tabular}{|c|c|c|c|c|c|}
\hline clustername & bootstrap & leafname & branchPath & medianOfDistances & sequencesperCluster \\
\hline 2495 & 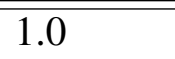 & "EPI_ISL_429531 & "6.526499999999994E-4 & 8.248999999999973E-5 & 50 \\
\hline 2495 & 1.0 & EPI_ISL_429540 & 6.086799999999995E-4 & 8.248999999999973E-5 & 50 \\
\hline 2495 & 1.0 & EPI_ISL_429493 & 6.408999999999996E-4 & 8.248999999999973E-5 & 50 \\
\hline 2495 & 1.0 & EPI_ISL_429563 & 6.086799999999995E-4 & 8.248999999999973E-5 & 50 \\
\hline 2495 & 1.0 & EPI_ISL_429130 & 6.430799999999995E-4 & 8.248999999999973E-5 & 50 \\
\hline 2495 & 1.0 & EPI_ISL_429321 & 7.140599999999997E-4 & 8.248999999999973E-5 & 50 \\
\hline 2495 & 1.0 & EPI_ISL_429527 & 6.868099999999994E-4 & 8.248999999999973E-5 & 50 \\
\hline 2495 & 1.0 & EPI_ISL_429463 & 6.796599999999996E-4 & 8.248999999999973E-5 & 50 \\
\hline 2495 & 1.0 & EPI_ISL_429467 & 6.938299999999993E-4 & 8.248999999999973E-5 & 50 \\
\hline 2495 & 1.0 & EPI_ISL_429322 & 6.086799999999995E-4 & 8.248999999999973E-5 & 50 \\
\hline 2495 & 1.0 & EPI_ISL_417682 & 6.780899999999996E-4 & 8.248999999999973E-5 & 50 \\
\hline 2495 & 1.0 & EPI_ISL_429336 & 6.496199999999994E-4 & 8.248999999999973E-5 & 50 \\
\hline 2495 & 1.0 & EPI_ISL_429307 & 6.086799999999995E-4 & 8.248999999999973E-5 & 50 \\
\hline 2495 & 1.0 & EPI_ISL_429552 & 6.086799999999995E-4 & 8.248999999999973E-5 & 50 \\
\hline 2495 & 1.0 & EPI_ISL_429506 & 6.086799999999995E-4 & 8.248999999999973E-5 & 50 \\
\hline 2495 & 1.0 & EPI_ISL_429132 & 7.051899999999997E-4 & 8.248999999999973E-5 & 50 \\
\hline 2495 & 1.0 & EPI_ISL_429293 & 6.824499999999995E-4 & 8.248999999999973E-5 & 50 \\
\hline 2495 & 1.0 & EPI_ISL_429477 & 6.086799999999995E-4 & 8.248999999999973E-5 & 50 \\
\hline 2495 & 1.0 & EPI_ISL_429134 & 7.182599999999994E-4 & 8.248999999999973E-5 & 50 \\
\hline 2495 & 1.0 & EPI_ISL_424622 & 6.889899999999994E-4 & 8.248999999999973E-5 & 50 \\
\hline 2495 & 1.0 & EPI_ISL_429297 & 6.796599999999996E-4 & 8.248999999999973E-5 & 50 \\
\hline 2495 & 1.0 & EPI_ISL_429519 & 6.086799999999995E-4 & 8.248999999999973E-5 & 50 \\
\hline 2495 & 1.0 & EPI_ISL_429521 & 6.387099999999996E-4 & 8.248999999999973E-5 & 50 \\
\hline 2495 & 1.0 & EPI_ISL_429285 & 6.517999999999994E-4 & 8.248999999999973E-5 & 50 \\
\hline 2495 & 1.0 & EPI_ISL_429488 & 6.086799999999995E-4 & 8.248999999999973E-5 & 50 \\
\hline 2495 & 1.0 & EPI_ISL_429304 & 6.824499999999995E-4 & 8.248999999999973E-5 & 50 \\
\hline
\end{tabular}


Table S4: Clusters identified using a genetic distance threshold within $1 \%$ of the distribution of patristic distances within the entire tree. The minimum percentile threshold that maximized the number of clusters was chosen as the optimal threshold by performing multiple clustering runs on randomly sampled patristic distance distributions (1 million for each run) in Phylopart v2 (46).

\begin{tabular}{|c|c|c|c|c|c|}
\hline clustername & bootstrap & leafname & branchPath & medianOfDistances & sequencesperCluster \\
\hline 2495 & 1.0 & EPI_ISL_429574 & 6.086799999999995E-4 & "8.248999999999973E-5 & 50 \\
\hline 2495 & 1.0 & EPI_ISL_429331 & 6.452599999999995E-4 & 8.248999999999973E-5 & 50 \\
\hline 2495 & 1.0 & EPI_ISL_429546 & 6.539799999999993E-4 & 8.248999999999973E-5 & 50 \\
\hline 2495 & 1.0 & EPI_ISL_429305 & 6.086799999999995E-4 & 8.248999999999973E-5 & 50 \\
\hline 2495 & 1.0 & EPI_ISL_429286 & 6.173999999999993E-4 & 8.248999999999973E-5 & 50 \\
\hline 2495 & 1.0 & EPI_ISL_429557 & 6.086799999999995E-4 & 8.248999999999973E-5 & 50 \\
\hline 2495 & 1.0 & EPI_ISL_429472 & 6.086799999999995E-4 & 8.248999999999973E-5 & 50 \\
\hline 2495 & 1.0 & EPI_ISL_429318 & 6.086799999999995E-4 & 8.248999999999973E-5 & 50 \\
\hline 2495 & 1.0 & EPI_ISL_429489 & 6.299999999999998E-4 & 8.248999999999973E-5 & 50 \\
\hline 2495 & 1.0 & EPI_ISL_429479 & 6.343599999999997E-4 & 8.248999999999973E-5 & 50 \\
\hline 2495 & 1.0 & EPI_ISL_429483 & 6.086799999999995E-4 & 8.248999999999973E-5 & 50 \\
\hline 2495 & 1.0 & EPI_ISL_415648 & 6.496199999999994E-4 & 8.248999999999973E-5 & 50 \\
\hline 2495 & 1.0 & EPI_ISL_424532 & $6.780899999999996 \mathrm{E}-4$ & 8.248999999999973E-5 & 50 \\
\hline 2495 & 1.0 & EPI_ISL_429556 & 6.539799999999993E-4 & 8.248999999999973E-5 & 50 \\
\hline 2495 & 1.0 & EPI_ISL_429562 & 6.086799999999995E-4 & 8.248999999999973E-5 & 50 \\
\hline 2495 & 1.0 & EPI_ISL_429543 & 6.086799999999995E-4 & 8.248999999999973E-5 & 50 \\
\hline 2495 & 1.0 & EPI_ISL_429496 & 6.086799999999995E-4 & 8.248999999999973E-5 & 50 \\
\hline 2495 & 1.0 & EPI_ISL_429289 & 6.477299999999995E-4 & 8.248999999999973E-5 & 50 \\
\hline 2495 & 1.0 & EPI_ISL_429485 & 6.086799999999995E-4 & 8.248999999999973E-5 & 50 \\
\hline 2495 & 1.0 & EPI_ISL_429481 & 6.086799999999995E-4 & 8.248999999999973E-5 & 50 \\
\hline 2495 & 1.0 & EPI_ISL_417690 & 7.613899999999994E-4 & 8.248999999999973E-5 & 50 \\
\hline 2495 & 1.0 & EPI_ISL_424400 & 6.780899999999996E-4 & 8.248999999999973E-5 & 50 \\
\hline 2495 & 1.0 & EPI_ISL_429131 & 6.086799999999995E-4 & 8.248999999999973E-5 & 50 \\
\hline 2597 & 1.0 & EPI_ISL_425263 & 6.387299999999996E-4 & $3.874999999999994 \mathrm{E}-5$ & 3 \\
\hline 2597 & 1.0 & EPI_ISL_423384 & 6.043399999999995E-4 & $3.874999999999994 \mathrm{E}-5$ & 3 \\
\hline 2597 & 1.0 & EPI_ISL_423383 & 6.021599999999996E-4 & 3.874999999999994E-5 & 3 \\
\hline
\end{tabular}


Table S4: Clusters identified using a genetic distance threshold within $1 \%$ of the distribution of patristic distances within the entire tree. The minimum percentile threshold that maximized the number of clusters was chosen as the optimal threshold by performing multiple clustering runs on randomly sampled patristic distance distributions (1 million for each run) in Phylopart v2 (46).

\begin{tabular}{|c|c|c|c|c|c|}
\hline clustername & bootstrap & leafname & branchPath & medianOfDistances & sequencesperCluster \\
\hline 2602 & 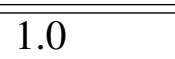 & "EPI_ISL_429656 & 6.021299999999995E-4 & 3.874999999999994E-5 & 2 \\
\hline 2602 & 1.0 & EPI_ISL_422609 & 6.365199999999996E-4 & 3.874999999999994E-5 & 2 \\
\hline 2607 & 1.0 & EPI_ISL_428892 & 6.343399999999996E-4 & 3.874999999999994E-5 & 3 \\
\hline 2607 & 1.0 & EPI_ISL_428902 & 6.343399999999996E-4 & 3.874999999999994E-5 & 3 \\
\hline 2607 & 1.0 & EPI_ISL_420579 & 5.999499999999996E-4 & 3.874999999999994E-5 & 3 \\
\hline 2616 & 1.0 & EPI_ISL_424311 & 6.759399999999995E-4 & 7.598999999999996E-5 & 2 \\
\hline 2616 & 1.0 & EPI_ISL_425912 & 6.043099999999995E-4 & 7.598999999999996E-5 & 2 \\
\hline 2645 & 1.0 & EPI_ISL_429569 & 6.002499999999997E-4 & 3.874999999999994E-5 & 2 \\
\hline 2645 & 1.0 & EPI_ISL_429464 & 6.346399999999997E-4 & 3.874999999999994E-5 & 2 \\
\hline 2690 & 1.0 & EPI_ISL_421503 & 6.799199999999995E-4 & 4.359999999999911E-6 & 2 \\
\hline 2690 & 1.0 & EPI_ISL_420054 & 6.799199999999995E-4 & 4.359999999999911E-6 & 2 \\
\hline 2693 & 1.0 & EPI_ISL_421300 & 6.771299999999995E-4 & 3.874999999999994E-5 & 2 \\
\hline 2693 & 1.0 & EPI_ISL_416523 & 6.427399999999995E-4 & 3.874999999999994E-5 & 2 \\
\hline 2696 & 1.0 & EPI_ISL_426884 & 6.771399999999996E-4 & 4.6029999999999856E-5 & 7 \\
\hline 2696 & 1.0 & EPI_ISL_422636 & 6.735099999999997E-4 & 4.6029999999999856E-5 & 7 \\
\hline 2696 & 1.0 & EPI_ISL_426887 & 7.121699999999996E-4 & 4.6029999999999856E-5 & 7 \\
\hline 2696 & 1.0 & EPI_ISL_425760 & 6.449199999999995E-4 & 4.6029999999999856E-5 & 7 \\
\hline 2696 & 1.0 & EPI_ISL_425692 & 6.449199999999995E-4 & 4.6029999999999856E-5 & 7 \\
\hline 2696 & 1.0 & EPI_ISL_417830 & 6.449199999999995E-4 & 4.6029999999999856E-5 & 7 \\
\hline 2696 & 1.0 & EPI_ISL_428923 & 6.793099999999995E-4 & $4.6029999999999856 \mathrm{E}-5$ & 7 \\
\hline 2709 & 1.0 & EPI_ISL_426668 & 6.733899999999996E-4 & 3.841999999999995E-5 & 2 \\
\hline 2709 & 1.0 & EPI_ISL_426654 & 7.074499999999997E-4 & $3.841999999999995 \mathrm{E}-5$ & 2 \\
\hline 2714 & 1.0 & EPI_ISL_419915 & 7.482599999999996E-4 & 4.359999999999911E-6 & 2 \\
\hline 2714 & 1.0 & EPI_ISL_426768 & 7.482599999999996E-4 & 4.359999999999911E-6 & 2 \\
\hline 2722 & 1.0 & EPI_ISL_425992 & 6.408899999999995E-4 & 0.0 & 5 \\
\hline 2722 & 1.0 & EPI_ISL_425906 & 6.408899999999995E-4 & 0.0 & 5 \\
\hline
\end{tabular}


Table S4: Clusters identified using a genetic distance threshold within $1 \%$ of the distribution of patristic distances within the entire tree. The minimum percentile threshold that maximized the number of clusters was chosen as the optimal threshold by performing multiple clustering runs on randomly sampled patristic distance distributions (1 million for each run) in Phylopart v2 (46).

\begin{tabular}{|c|c|c|c|c|c|}
\hline clustername & bootstrap & leafname & branchPath & medianOfDistances & sequencesperCluster \\
\hline 2722 & 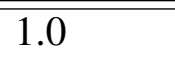 & "EPI_ISL_425907 & 6.408899999999995E-4 & 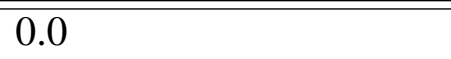 & $\overline{5}$ \\
\hline 2722 & 1.0 & EPI_ISL_425866 & 6.752999999999996E-4 & 0.0 & 5 \\
\hline 2722 & 1.0 & EPI_ISL_426003 & 6.408899999999995E-4 & 0.0 & 5 \\
\hline 2733 & 1.0 & EPI_ISL_414630 & 6.818199999999994E-4 & 7.594999999999998E-5 & 3 \\
\hline 2733 & 1.0 & EPI_ISL_428360 & 6.796399999999995E-4 & 7.594999999999998E-5 & 3 \\
\hline 2733 & 1.0 & EPI_ISL_418237 & 7.534099999999995E-4 & 7.594999999999998E-5 & 3 \\
\hline 2747 & 1.0 & EPI_ISL_428355 & 6.818299999999995E-4 & 0.0 & 5 \\
\hline 2747 & 1.0 & EPI_ISL_428351 & 6.474299999999994E-4 & 0.0 & 5 \\
\hline 2747 & 1.0 & EPI_ISL_428356 & 6.474299999999994E-4 & 0.0 & 5 \\
\hline 2747 & 1.0 & EPI_ISL_416498 & 6.474299999999994E-4 & 0.0 & 5 \\
\hline 2747 & 1.0 & EPI_ISL_428357 & 6.474299999999994E-4 & 0.0 & 5 \\
\hline 2772 & 1.0 & EPI_ISL_421332 & 5.999499999999996E-4 & 4.0939999999999943E-5 & 15 \\
\hline 2772 & 1.0 & EPI_ISL_423011 & 6.321699999999997E-4 & 4.0939999999999943E-5 & 15 \\
\hline 2772 & 1.0 & EPI_ISL_425150 & 6.321699999999997E-4 & 4.0939999999999943E-5 & 15 \\
\hline 2772 & 1.0 & EPI_ISL_425149 & 5.999499999999996E-4 & 4.0939999999999943E-5 & 15 \\
\hline 2772 & 1.0 & EPI_ISL_421302 & 6.321699999999997E-4 & 4.0939999999999943E-5 & 15 \\
\hline 2772 & 1.0 & EPI_ISL_421287 & 5.999499999999996E-4 & 4.0939999999999943E-5 & 15 \\
\hline 2772 & 1.0 & EPI_ISL_427529 & 5.999499999999996E-4 & 4.0939999999999943E-5 & 15 \\
\hline 2772 & 1.0 & EPI_ISL_424945 & $6.259299999999998 \mathrm{E}-4$ & 4.0939999999999943E-5 & 15 \\
\hline 2772 & 1.0 & EPI_ISL_425159 & 7.037699999999997E-4 & 4.0939999999999943E-5 & 15 \\
\hline 2772 & 1.0 & EPI_ISL_426092 & 6.278999999999998E-4 & 4.0939999999999943E-5 & 15 \\
\hline 2772 & 1.0 & EPI_ISL_416642 & 5.999499999999996E-4 & 4.0939999999999943E-5 & 15 \\
\hline 2772 & 1.0 & EPI_ISL_416491 & 5.999499999999996E-4 & 4.0939999999999943E-5 & 15 \\
\hline 2772 & 1.0 & EPI_ISL_416492 & 5.999499999999996E-4 & 4.0939999999999943E-5 & 15 \\
\hline 2772 & 1.0 & EPI_ISL_421311 & 6.321699999999997E-4 & 4.0939999999999943E-5 & 15 \\
\hline 2772 & 1.0 & EPI_ISL_422994 & 5.999499999999996E-4 & 4.0939999999999943E-5 & 15 \\
\hline
\end{tabular}


Table S4: Clusters identified using a genetic distance threshold within $1 \%$ of the distribution of patristic distances within the entire tree. The minimum percentile threshold that maximized the number of clusters was chosen as the optimal threshold by performing multiple clustering runs on randomly sampled patristic distance distributions (1 million for each run) in Phylopart v2 (46).

\begin{tabular}{|c|c|c|c|c|c|}
\hline clustername & bootstrap & leafname & branchPath & medianOfDistances & sequencesperCluster \\
\hline 2819 & 1.0 & "EPI_ISL_420573 & 6.321799999999998E-4 & 7.969999999999982E-5 & 10 \\
\hline 2819 & 1.0 & EPI_ISL_427592 & 5.955999999999997E-4 & 7.969999999999982E-5 & 10 \\
\hline 2819 & 1.0 & EPI_ISL_429129 & 7.015899999999999E-4 & 7.969999999999982E-5 & 10 \\
\hline 2819 & 1.0 & EPI_ISL_419702 & 6.321799999999998E-4 & 7.969999999999982E-5 & 10 \\
\hline 2819 & 1.0 & EPI_ISL_421728 & $6.665799999999998 \mathrm{E}-4$ & 7.969999999999982E-5 & 10 \\
\hline 2819 & 1.0 & EPI_ISL_428772 & $6.256399999999999 \mathrm{E}-4$ & 7.969999999999982E-5 & 10 \\
\hline 2819 & 1.0 & EPI_ISL_421675 & $6.321799999999998 \mathrm{E}-4$ & 7.969999999999982E-5 & 10 \\
\hline 2819 & 1.0 & EPI_ISL_418041 & 5.955999999999997E-4 & 7.969999999999982E-5 & 10 \\
\hline 2819 & 1.0 & EPI_ISL_427607 & $6.299999999999998 \mathrm{E}-4$ & 7.969999999999982E-5 & 10 \\
\hline 2819 & 1.0 & EPI_ISL_426128 & 6.607399999999999E-4 & 7.969999999999982E-5 & 10 \\
\hline 2846 & 1.0 & EPI_ISL_427491 & 5.868699999999999E-4 & 3.874999999999994E-5 & 2 \\
\hline 2846 & 1.0 & EPI_ISL_429641 & 6.212599999999999E-4 & 3.874999999999994E-5 & 2 \\
\hline 2861 & 1.0 & EPI_ISL_419864 & 6.628199999999998E-4 & 3.874999999999994E-5 & 3 \\
\hline 2861 & 1.0 & EPI_ISL_426683 & 6.993899999999998E-4 & 3.874999999999994E-5 & 3 \\
\hline 2861 & 1.0 & EPI_ISL_419844 & 6.649999999999998E-4 & 3.874999999999994E-5 & 3 \\
\hline 2878 & 1.0 & EPI_ISL_424215 & $6.621999999999998 \mathrm{E}-4$ & 7.531999999999981E-5 & 6 \\
\hline 2878 & 1.0 & EPI_ISL_426100 & $6.321699999999997 \mathrm{E}-4$ & 7.531999999999981E-5 & 6 \\
\hline 2878 & 1.0 & EPI_ISL_424294 & 7.015899999999998E-4 & 7.531999999999981E-5 & 6 \\
\hline 2878 & 1.0 & EPI_ISL_424166 & $6.709199999999998 \mathrm{E}-4$ & 7.531999999999981E-5 & 6 \\
\hline 2878 & 1.0 & EPI_ISL_427212 & 7.031299999999997E-4 & 7.531999999999981E-5 & 6 \\
\hline 2878 & 1.0 & EPI_ISL_424263 & 6.321699999999997E-4 & 7.531999999999981E-5 & 6 \\
\hline 2901 & 1.0 & EPI_ISL_420056 & 6.190099999999997E-4 & 7.592999999999988E-5 & 2 \\
\hline 2901 & 1.0 & EPI_ISL_421509 & 6.905799999999998E-4 & 7.592999999999988E-5 & 2 \\
\hline 2960 & 1.0 & EPI_ISL_425643 & 6.598699999999999E-4 & 4.359999999999911E-6 & 2 \\
\hline 2960 & 1.0 & EPI_ISL_428365 & 6.598699999999999E-4 & 4.359999999999911E-6 & 2 \\
\hline 2980 & 1.0 & EPI_ISL_422419 & $6.661099999999997 \mathrm{E}-4$ & 3.874999999999994E-5 & 2 \\
\hline
\end{tabular}


Table S4: Clusters identified using a genetic distance threshold within $1 \%$ of the distribution of patristic distances within the entire tree. The minimum percentile threshold that maximized the number of clusters was chosen as the optimal threshold by performing multiple clustering runs on randomly sampled patristic distance distributions (1 million for each run) in Phylopart v2 (46).

\begin{tabular}{|c|c|c|c|c|c|}
\hline clustername & bootstrap & leafname & branchPath & medianOfDistances & sequencesperCluster \\
\hline 2980 & 1.0 & "EPI_ISL_426632 & 6.317199999999997E-4 & 3.874999999999994E-5 & $\overline{2}$ \\
\hline 3010 & 1.0 & EPI_ISL_420150 & 6.299599999999997E-4 & 4.359999999999911E-6 & 4 \\
\hline 3010 & 1.0 & EPI_ISL_429262 & 6.299599999999997E-4 & 4.359999999999911E-6 & 4 \\
\hline 3010 & 1.0 & EPI_ISL_429551 & $6.299599999999997 \mathrm{E}-4$ & 4.359999999999911E-6 & 4 \\
\hline 3010 & 1.0 & EPI_ISL_425758 & $6.299599999999997 \mathrm{E}-4$ & 4.359999999999911E-6 & 4 \\
\hline 3018 & 1.0 & EPI_ISL_415477 & 6.670099999999997E-4 & $7.361 \mathrm{E}-5$ & 2 \\
\hline 3018 & 1.0 & EPI_ISL_415525 & $6.665399999999996 \mathrm{E}-4$ & $7.361 \mathrm{E}-5$ & 2 \\
\hline 3042 & 1.0 & EPI_ISL_421378 & 6.430599999999994E-4 & 8.028999999999979E-5 & 11 \\
\hline 3042 & 1.0 & EPI_ISL_421635 & 6.430599999999994E-4 & 8.028999999999979E-5 & 11 \\
\hline 3042 & 1.0 & EPI_ISL_422546 & 7.483299999999995E-4 & 8.028999999999979E-5 & 11 \\
\hline 3042 & 1.0 & EPI_ISL_426317 & 6.430599999999994E-4 & 8.028999999999979E-5 & 11 \\
\hline 3042 & 1.0 & EPI_ISL_420588 & 6.430599999999994E-4 & 8.028999999999979E-5 & 11 \\
\hline 3042 & 1.0 & EPI_ISL_421630 & 7.074999999999997E-4 & 8.028999999999979E-5 & 11 \\
\hline 3042 & 1.0 & EPI_ISL_427547 & 6.430599999999994E-4 & 8.028999999999979E-5 & 11 \\
\hline 3042 & 1.0 & EPI_ISL_424931 & 7.102699999999996E-4 & 8.028999999999979E-5 & 11 \\
\hline 3042 & 1.0 & EPI_ISL_422519 & $6.752799999999996 \mathrm{E}-4$ & 8.028999999999979E-5 & 11 \\
\hline 3042 & 1.0 & EPI_ISL_422534 & $6.752799999999996 \mathrm{E}-4$ & 8.028999999999979E-5 & 11 \\
\hline 3042 & 1.0 & EPI_ISL_424930 & 7.102699999999996E-4 & 8.028999999999979E-5 & 11 \\
\hline 3076 & 1.0 & EPI_ISL_418964 & 7.395399999999997E-4 & 4.359999999999911E-6 & 2 \\
\hline 3076 & 1.0 & EPI_ISL_418963 & 7.395399999999997E-4 & 4.359999999999911E-6 & 2 \\
\hline 3079 & 1.0 & EPI_ISL_427799 & 5.934099999999997E-4 & 3.874999999999994E-5 & 3 \\
\hline 3079 & 1.0 & EPI_ISL_420238 & $6.277999999999998 \mathrm{E}-4$ & 3.874999999999994E-5 & 3 \\
\hline 3079 & 1.0 & EPI_ISL_427795 & 5.934099999999997E-4 & 3.874999999999994E-5 & 3 \\
\hline 3089 & 1.0 & EPI_ISL_420301 & $6.212599999999999 \mathrm{E}-4$ & 3.874999999999994E-5 & 2 \\
\hline 3089 & 1.0 & EPI_ISL_421721 & $5.868699999999999 \mathrm{E}-4$ & 3.874999999999994E-5 & 2 \\
\hline 3093 & 1.0 & EPI_ISL_424306 & 5.846899999999999E-4 & 4.359999999999911E-6 & 2 \\
\hline
\end{tabular}


Table S4: Clusters identified using a genetic distance threshold within $1 \%$ of the distribution of patristic distances within the entire tree. The minimum percentile threshold that maximized the number of clusters was chosen as the optimal threshold by performing multiple clustering runs on randomly sampled patristic distance distributions (1 million for each run) in Phylopart v2 (46).

\begin{tabular}{|c|c|c|c|c|c|}
\hline clustername & bootstrap & leafname & branchPath & medianOfDistances & sequencesperCluster \\
\hline 3093 & 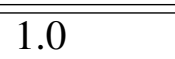 & "EPI_ISL_424339 & 5.846899999999999E-4 & "4.359999999999911E-6 & 2 \\
\hline 3103 & 1.0 & EPI_ISL_415649 & 6.299699999999999E-4 & 4.12199999999998E-5 & 3 \\
\hline 3103 & 1.0 & EPI_ISL_413572 & 5.955899999999998E-4 & 4.12199999999998E-5 & 3 \\
\hline 3103 & 1.0 & EPI_ISL_420322 & 6.280899999999999E-4 & 4.12199999999998E-5 & 3 \\
\hline 3111 & 1.0 & EPI_ISL_426028 & 6.672299999999998E-4 & $7.600000000000011 E-5$ & 2 \\
\hline 3111 & 1.0 & EPI_ISL_417707 & 5.955899999999997E-4 & $7.600000000000011 E-5$ & 2 \\
\hline 3114 & 1.0 & EPI_ISL_429482 & 6.302799999999998E-4 & $7.532000000000003 E-5$ & 3 \\
\hline 3114 & 1.0 & EPI_ISL_429480 & 6.324499999999997E-4 & $7.532000000000003 \mathrm{E}-5$ & 3 \\
\hline 3114 & 1.0 & EPI_ISL_429581 & 6.324399999999997E-4 & $7.532000000000003 E-5$ & 3 \\
\hline 3123 & 1.0 & EPI_ISL_427476 & 5.890499999999998E-4 & 3.874999999999994E-5 & 3 \\
\hline 3123 & 1.0 & EPI_ISL_418974 & $6.234399999999998 \mathrm{E}-4$ & $3.874999999999994 \mathrm{E}-5$ & 3 \\
\hline 3123 & 1.0 & EPI_ISL_418200 & 5.868699999999999E-4 & $3.874999999999994 \mathrm{E}-5$ & 3 \\
\hline 3136 & 1.0 & EPI_ISL_426124 & 5.890499999999998E-4 & $3.876999999999982 \mathrm{E}-5$ & 2 \\
\hline 3136 & 1.0 & EPI_ISL_427554 & 6.234599999999998E-4 & 3.876999999999982E-5 & 2 \\
\hline 3140 & 1.0 & EPI_ISL_426979 & 7.355999999999998E-4 & 4.359999999999911E-6 & 2 \\
\hline 3140 & 1.0 & EPI_ISL_429013 & 7.355999999999998E-4 & 4.359999999999911E-6 & 2 \\
\hline 3145 & 1.0 & EPI_ISL_426086 & 6.266799999999998E-4 & 3.951999999999992E-5 & 3 \\
\hline 3145 & 1.0 & EPI_ISL_415481 & 5.915199999999998E-4 & 3.951999999999992E-5 & 3 \\
\hline 3145 & 1.0 & EPI_ISL_424330 & 5.915199999999998E-4 & 3.951999999999992E-5 & 3 \\
\hline 3198 & 1.0 & EPI_ISL_421316 & 6.913899999999992E-4 & $7.592999999999988 \mathrm{E}-5$ & 2 \\
\hline 3198 & 1.0 & EPI_ISL_421312 & 7.629599999999993E-4 & $7.592999999999988 \mathrm{E}-5$ & 2 \\
\hline 3201 & 1.0 & EPI_ISL_426536 & 6.520399999999992E-4 & $3.874999999999994 \mathrm{E}-5$ & 2 \\
\hline 3201 & 1.0 & EPI_ISL_426526 & 6.864299999999993E-4 & $3.874999999999994 \mathrm{E}-5$ & 2 \\
\hline 3208 & 1.0 & EPI_ISL_418893 & 6.755299999999995E-4 & 8.029999999999995E-5 & 7 \\
\hline 3208 & 1.0 & EPI_ISL_417376 & 6.520399999999992E-4 & 8.029999999999995E-5 & 7 \\
\hline 3208 & 1.0 & EPI_ISL_423028 & 6.777099999999994E-4 & 8.029999999999995E-5 & 7 \\
\hline
\end{tabular}


Table S4: Clusters identified using a genetic distance threshold within $1 \%$ of the distribution of patristic distances within the entire tree. The minimum percentile threshold that maximized the number of clusters was chosen as the optimal threshold by performing multiple clustering runs on randomly sampled patristic distance distributions (1 million for each run) in Phylopart v2 (46).

\begin{tabular}{|c|c|c|c|c|c|}
\hline clustername & bootstrap & leafname & branchPath & medianOfDistances & sequencesperCluster \\
\hline 3208 & 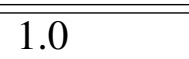 & "EPI_ISL_426627 & 7.550899999999994E-4 & "8.029999999999995E-5 & $\overline{77}$ \\
\hline 3208 & 1.0 & EPI_ISL_418036 & 6.498599999999993E-4 & 8.029999999999995E-5 & 7 \\
\hline 3208 & 1.0 & EPI_ISL_428335 & 6.520399999999992E-4 & 8.029999999999995E-5 & 7 \\
\hline 3208 & 1.0 & EPI_ISL_424868 & 7.170799999999994E-4 & 8.029999999999995E-5 & 7 \\
\hline 3222 & 1.0 & EPI_ISL_420814 & 6.476799999999993E-4 & 4.341999999999996E-5 & 6 \\
\hline 3222 & 1.0 & EPI_ISL_430048 & 6.411399999999995E-4 & 4.341999999999996E-5 & 6 \\
\hline 3222 & 1.0 & EPI_ISL_420815 & 6.801999999999994E-4 & 4.341999999999996E-5 & 6 \\
\hline 3222 & 1.0 & EPI_ISL_420824 & 6.801999999999994E-4 & 4.341999999999996E-5 & 6 \\
\hline 3222 & 1.0 & EPI_ISL_421563 & 6.801999999999994E-4 & 4.341999999999996E-5 & 6 \\
\hline 3222 & 1.0 & EPI_ISL_417971 & 6.476799999999993E-4 & 4.341999999999996E-5 & 6 \\
\hline 3236 & 1.0 & EPI_ISL_418051 & 6.498599999999993E-4 & 4.310999999999985E-5 & 4 \\
\hline 3236 & 1.0 & EPI_ISL_427284 & $6.842499999999993 \mathrm{E}-4$ & 4.310999999999985E-5 & 4 \\
\hline 3236 & 1.0 & EPI_ISL_427283 & 6.842499999999993E-4 & 4.310999999999985E-5 & 4 \\
\hline 3236 & 1.0 & EPI_ISL_418049 & 6.498599999999993E-4 & 4.310999999999985E-5 & 4 \\
\hline 3259 & 1.0 & EPI_ISL_420810 & 8.021599999999991E-4 & 4.359999999999911E-6 & 2 \\
\hline 3259 & 1.0 & EPI_ISL_420811 & 8.021599999999991E-4 & 4.359999999999911E-6 & 2 \\
\hline 3272 & 1.0 & EPI_ISL_426485 & 6.454999999999994E-4 & 4.359999999999911E-6 & 2 \\
\hline 3272 & 1.0 & EPI_ISL_426541 & 6.454999999999994E-4 & 4.359999999999911E-6 & 2 \\
\hline 3277 & 1.0 & EPI_ISL_426523 & 6.368099999999996E-4 & $7.602 \mathrm{E}-5$ & 3 \\
\hline 3277 & 1.0 & EPI_ISL_426500 & 6.368099999999996E-4 & $7.602 \mathrm{E}-5$ & 3 \\
\hline 3277 & 1.0 & EPI_ISL_426521 & 7.084699999999997E-4 & $7.602 \mathrm{E}-5$ & 3 \\
\hline 3282 & 1.0 & EPI_ISL_427622 & 6.367699999999996E-4 & 7.361999999999928E-5 & 52 \\
\hline 3282 & 1.0 & EPI_ISL_416661 & 6.367699999999996E-4 & 7.361999999999928E-5 & 52 \\
\hline 3282 & 1.0 & EPI_ISL_418037 & 6.367699999999996E-4 & 7.361999999999928E-5 & 52 \\
\hline 3282 & 1.0 & EPI_ISL_427169 & 6.367699999999996E-4 & 7.361999999999928E-5 & 52 \\
\hline 3282 & 1.0 & EPI_ISL_424315 & 8.113699999999991E-4 & 7.361999999999928E-5 & 52 \\
\hline
\end{tabular}


Table S4: Clusters identified using a genetic distance threshold within $1 \%$ of the distribution of patristic distances within the entire tree. The minimum percentile threshold that maximized the number of clusters was chosen as the optimal threshold by performing multiple clustering runs on randomly sampled patristic distance distributions (1 million for each run) in Phylopart v2 (46).

\begin{tabular}{|c|c|c|c|c|c|}
\hline clustername & bootstrap & leafname & branchPath & medianOfDistances & sequencesperCluster \\
\hline 3282 & 1.0 & "EPI_ISL_424321 & 6.367699999999996E-4 & 7.361999999999928E-5 & 52 \\
\hline 3282 & 1.0 & EPI_ISL_427221 & 6.558799999999994E-4 & 7.361999999999928E-5 & 52 \\
\hline 3282 & 1.0 & EPI_ISL_420825 & 6.520299999999993E-4 & 7.361999999999928E-5 & 52 \\
\hline 3282 & 1.0 & EPI_ISL_426074 & 7.397999999999998E-4 & 7.361999999999928E-5 & 52 \\
\hline 3282 & 1.0 & EPI_ISL_426060 & $6.367699999999996 \mathrm{E}-4$ & 7.361999999999928E-5 & 52 \\
\hline 3282 & 1.0 & EPI_ISL_426058 & $6.798899999999994 \mathrm{E}-4$ & 7.361999999999928E-5 & 52 \\
\hline 3282 & 1.0 & EPI_ISL_429601 & $6.367699999999996 \mathrm{E}-4$ & 7.361999999999928E-5 & 52 \\
\hline 3282 & 1.0 & EPI_ISL_424319 & 6.563899999999992E-4 & 7.361999999999928E-5 & 52 \\
\hline 3282 & 1.0 & EPI_ISL_429027 & 6.367699999999996E-4 & 7.361999999999928E-5 & 52 \\
\hline 3282 & 1.0 & EPI_ISL_424354 & $6.781899999999988 \mathrm{E}-4$ & 7.361999999999928E-5 & 52 \\
\hline 3282 & 1.0 & EPI_ISL_424318 & 6.367699999999996E-4 & 7.361999999999928E-5 & 52 \\
\hline 3282 & 1.0 & EPI_ISL_429009 & 6.367699999999996E-4 & 7.361999999999928E-5 & 52 \\
\hline 3282 & 1.0 & EPI_ISL_426067 & $6.411299999999995 \mathrm{E}-4$ & 7.361999999999928E-5 & 52 \\
\hline 3282 & 1.0 & EPI_ISL_424225 & 6.842299999999994E-4 & 7.361999999999928E-5 & 52 \\
\hline 3282 & 1.0 & EPI_ISL_429648 & 7.131999999999996E-4 & 7.361999999999928E-5 & 52 \\
\hline 3282 & 1.0 & EPI_ISL_426077 & 6.367699999999996E-4 & 7.361999999999928E-5 & 52 \\
\hline 3282 & 1.0 & EPI_ISL_424989 & 7.046899999999995E-4 & 7.361999999999928E-5 & 52 \\
\hline 3282 & 1.0 & EPI_ISL_427170 & $6.367699999999996 \mathrm{E}-4$ & 7.361999999999928E-5 & 52 \\
\hline 3282 & 1.0 & EPI_ISL_425146 & 7.38849999999999E-4 & 7.361999999999928E-5 & 52 \\
\hline 3282 & 1.0 & EPI_ISL_417345 & 6.433099999999995E-4 & 7.361999999999928E-5 & 52 \\
\hline 3282 & 1.0 & EPI_ISL_424327 & 6.367699999999996E-4 & 7.361999999999928E-5 & 52 \\
\hline 3282 & 1.0 & EPI_ISL_426061 & 6.798699999999995E-4 & 7.361999999999928E-5 & 52 \\
\hline 3282 & 1.0 & EPI_ISL_422972 & 6.454899999999994E-4 & 7.361999999999928E-5 & 52 \\
\hline 3282 & 1.0 & EPI_ISL_426063 & 8.54859999999999E-4 & 7.361999999999928E-5 & 52 \\
\hline 3282 & 1.0 & EPI_ISL_426072 & 7.016699999999991E-4 & 7.361999999999928E-5 & 52 \\
\hline 3282 & 1.0 & EPI_ISL_429003 & 6.367699999999996E-4 & 7.361999999999928E-5 & 52 \\
\hline
\end{tabular}


Table S4: Clusters identified using a genetic distance threshold within $1 \%$ of the distribution of patristic distances within the entire tree. The minimum percentile threshold that maximized the number of clusters was chosen as the optimal threshold by performing multiple clustering runs on randomly sampled patristic distance distributions (1 million for each run) in Phylopart v2 (46).

\begin{tabular}{|c|c|c|c|c|c|}
\hline clustername & bootstrap & leafname & branchPath & medianOfDistances & sequencesperCluster \\
\hline 3282 & 1.0 & "EPI_ISL_426073 & 7.257699999999993E-4 & 7.361999999999928E-5 & 52 \\
\hline 3282 & 1.0 & EPI_ISL_424176 & 7.04099999999999E-4 & 7.361999999999928E-5 & 52 \\
\hline 3282 & 1.0 & EPI_ISL_418072 & 6.760099999999988E-4 & 7.361999999999928E-5 & 52 \\
\hline 3282 & 1.0 & EPI_ISL_426069 & 6.973099999999992E-4 & 7.361999999999928E-5 & 52 \\
\hline 3282 & 1.0 & EPI_ISL_424320 & 6.875499999999994E-4 & 7.361999999999928E-5 & 52 \\
\hline 3282 & 1.0 & EPI_ISL_426127 & $6.755099999999996 \mathrm{E}-4$ & 7.361999999999928E-5 & 52 \\
\hline 3282 & 1.0 & EPI_ISL_424307 & $6.367699999999996 \mathrm{E}-4$ & 7.361999999999928E-5 & 52 \\
\hline 3282 & 1.0 & EPI_ISL_430028 & 6.932799999999992E-4 & 7.361999999999928E-5 & 52 \\
\hline 3282 & 1.0 & EPI_ISL_426831 & 7.125799999999988E-4 & 7.361999999999928E-5 & 52 \\
\hline 3282 & 1.0 & EPI_ISL_426064 & 7.323499999999991E-4 & 7.361999999999928E-5 & 52 \\
\hline 3282 & 1.0 & EPI_ISL_428992 & 7.148699999999995E-4 & 7.361999999999928E-5 & 52 \\
\hline 3282 & 1.0 & EPI_ISL_426066 & 6.367699999999996E-4 & 7.361999999999928E-5 & 52 \\
\hline 3282 & 1.0 & EPI_ISL_429599 & 7.087599999999995E-4 & 7.361999999999928E-5 & 52 \\
\hline 3282 & 1.0 & EPI_ISL_426065 & 6.367699999999996E-4 & 7.361999999999928E-5 & 52 \\
\hline 3282 & 1.0 & EPI_ISL_429042 & 7.447799999999989E-4 & 7.361999999999928E-5 & 52 \\
\hline 3282 & 1.0 & EPI_ISL_426071 & 6.411299999999996E-4 & 7.361999999999928E-5 & 52 \\
\hline 3282 & 1.0 & EPI_ISL_417932 & 6.367699999999996E-4 & 7.361999999999928E-5 & 52 \\
\hline 3282 & 1.0 & EPI_ISL_429610 & 6.475699999999994E-4 & 7.361999999999928E-5 & 52 \\
\hline 3282 & 1.0 & EPI_ISL_418845 & 6.716499999999989E-4 & 7.361999999999928E-5 & 52 \\
\hline 3282 & 1.0 & EPI_ISL_424322 & 6.367699999999996E-4 & 7.361999999999928E-5 & 52 \\
\hline 3282 & 1.0 & EPI_ISL_429037 & 7.192299999999994E-4 & 7.361999999999928E-5 & 52 \\
\hline 3387 & 1.0 & EPI_ISL_426535 & 7.036899999999998E-4 & 4.0929999999999786E-5 & 5 \\
\hline 3387 & 1.0 & EPI_ISL_426505 & 7.036899999999998E-4 & 4.0929999999999786E-5 & 5 \\
\hline 3387 & 1.0 & EPI_ISL_426520 & 7.058699999999997E-4 & 4.0929999999999786E-5 & 5 \\
\hline 3387 & 1.0 & EPI_ISL_426501 & 6.714799999999997E-4 & $4.0929999999999786 \mathrm{E}-5$ & 5 \\
\hline 3387 & 1.0 & EPI_ISL_426522 & 7.058699999999997E-4 & 4.0929999999999786E-5 & 5 \\
\hline
\end{tabular}


Table S4: Clusters identified using a genetic distance threshold within $1 \%$ of the distribution of patristic distances within the entire tree. The minimum percentile threshold that maximized the number of clusters was chosen as the optimal threshold by performing multiple clustering runs on randomly sampled patristic distance distributions (1 million for each run) in Phylopart v2 (46).

\begin{tabular}{|c|c|c|c|c|c|}
\hline clustername & bootstrap & leafname & branchPath & medianOfDistances & sequencesperCluster \\
\hline 3399 & 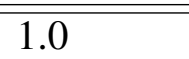 & "EPI_ISL_429026 & 6.671399999999998E-4 & 7.354999999999992E-5 & $\overline{2}$ \\
\hline 3399 & 1.0 & EPI_ISL_428396 & 6.675699999999997E-4 & 7.354999999999992E-5 & 2 \\
\hline 3404 & 1.0 & EPI_ISL_427073 & 6.695199999999996E-4 & 4.856999999999973E-5 & 6 \\
\hline 3404 & 1.0 & EPI_ISL_426928 & 6.704499999999996E-4 & 4.856999999999973E-5 & 6 \\
\hline 3404 & 1.0 & EPI_ISL_426756 & $6.393199999999995 \mathrm{E}-4$ & 4.856999999999973E-5 & 6 \\
\hline 3404 & 1.0 & EPI_ISL_426805 & 7.445399999999996E-4 & 4.856999999999973E-5 & 6 \\
\hline 3404 & 1.0 & EPI_ISL_419951 & $6.393199999999995 \mathrm{E}-4$ & 4.856999999999973E-5 & 6 \\
\hline 3404 & 1.0 & EPI_ISL_426991 & 6.349599999999996E-4 & 4.856999999999973E-5 & 6 \\
\hline 3426 & 1.0 & EPI_ISL_420312 & 6.343199999999996E-4 & 4.359999999999911E-6 & 3 \\
\hline 3426 & 1.0 & EPI_ISL_429277 & 6.343199999999996E-4 & 4.359999999999911E-6 & 3 \\
\hline 3426 & 1.0 & EPI_ISL_429391 & 6.343199999999996E-4 & 4.359999999999911E-6 & 3 \\
\hline 3439 & 1.0 & EPI_ISL_429460 & $5.890399999999999 \mathrm{E}-4$ & $6.539999999999758 \mathrm{E}-6$ & 4 \\
\hline 3439 & 1.0 & EPI_ISL_429271 & 5.890399999999999E-4 & $6.539999999999758 \mathrm{E}-6$ & 4 \\
\hline 3439 & 1.0 & EPI_ISL_418396 & 5.868599999999999E-4 & 6.539999999999758E-6 & 4 \\
\hline 3439 & 1.0 & EPI_ISL_427673 & 5.890399999999999E-4 & 6.539999999999758E-6 & 4 \\
\hline 3470 & 1.0 & EPI_ISL_421551 & 5.955899999999997E-4 & 4.359999999999911E-6 & 2 \\
\hline 3470 & 1.0 & EPI_ISL_422740 & 5.955899999999997E-4 & 4.359999999999911E-6 & 2 \\
\hline 3473 & 1.0 & EPI_ISL_430035 & $6.324799999999997 \mathrm{E}-4$ & 7.811999999999988E-5 & 3 \\
\hline 3473 & 1.0 & EPI_ISL_417976 & $6.671699999999997 \mathrm{E}-4$ & 7.811999999999988E-5 & 3 \\
\hline 3473 & 1.0 & EPI_ISL_419806 & $5.977699999999996 \mathrm{E}-4$ & 7.811999999999988E-5 & 3 \\
\hline 3478 & 1.0 & EPI_ISL_421600 & 5.912299999999998E-4 & 3.874999999999994E-5 & 2 \\
\hline 3478 & 1.0 & EPI_ISL_419704 & $6.256199999999998 \mathrm{E}-4$ & 3.874999999999994E-5 & 2 \\
\hline 3507 & 1.0 & EPI_ISL_426499 & 7.023699999999998E-4 & 7.612999999999999E-5 & 2 \\
\hline 3507 & 1.0 & EPI_ISL_426507 & 6.305999999999997E-4 & 7.612999999999999E-5 & 2 \\
\hline 3511 & 0.988 & EPI_ISL_417701 & 5.911799999999997E-4 & 4.359999999999911E-6 & 3 \\
\hline 3511 & 0.988 & EPI_ISL_427613 & 5.911799999999997E-4 & 4.359999999999911E-6 & 3 \\
\hline
\end{tabular}


Table S4: Clusters identified using a genetic distance threshold within $1 \%$ of the distribution of patristic distances within the entire tree. The minimum percentile threshold that maximized the number of clusters was chosen as the optimal threshold by performing multiple clustering runs on randomly sampled patristic distance distributions (1 million for each run) in Phylopart v2 (46).

\begin{tabular}{|c|c|c|c|c|c|}
\hline clustername & bootstrap & leafname & branchPath & medianOfDistances & sequencesperCluster \\
\hline 3511 & 0.988 & "EPI_ISL_427603 & "5.911799999999997E-4 & 4.359999999999911E-6 & 3 \\
\hline 3522 & 1.0 & EPI_ISL_418646 & 6.364399999999996E-4 & 8.719999999999822E-6 & 9 \\
\hline 3522 & 1.0 & EPI_ISL_418666 & 6.342599999999997E-4 & 8.719999999999822E-6 & 9 \\
\hline 3522 & 1.0 & EPI_ISL_427344 & $6.298999999999998 \mathrm{E}-4$ & 8.719999999999822E-6 & 9 \\
\hline 3522 & 1.0 & EPI_ISL_420085 & 6.364399999999996E-4 & 8.719999999999822E-6 & 9 \\
\hline 3522 & 1.0 & EPI_ISL_417020 & 6.364399999999996E-4 & 8.719999999999822E-6 & 9 \\
\hline 3522 & 1.0 & EPI_ISL_418636 & 6.320799999999997E-4 & 8.719999999999822E-6 & 9 \\
\hline 3522 & 1.0 & EPI_ISL_424637 & 6.364399999999996E-4 & 8.719999999999822E-6 & 9 \\
\hline 3522 & 1.0 & EPI_ISL_421194 & 6.708299999999996E-4 & 8.719999999999822E-6 & 9 \\
\hline 3522 & 1.0 & EPI_ISL_421182 & 6.364399999999996E-4 & 8.719999999999822E-6 & 9 \\
\hline 3544 & 1.0 & EPI_ISL_427519 & 6.240399999999999E-4 & 4.359999999999911E-6 & 2 \\
\hline 3544 & 1.0 & EPI_ISL_421593 & 6.240399999999999E-4 & 4.359999999999911E-6 & 2 \\
\hline 3687 & 1.0 & EPI_ISL_427498 & 7.531999999999988E-4 & 4.359999999999911E-6 & 2 \\
\hline 3687 & 1.0 & EPI_ISL_421632 & 7.531999999999988E-4 & 4.359999999999911E-6 & 2 \\
\hline 3691 & 1.0 & EPI_ISL_420590 & 7.83219999999999E-4 & $3.874 \mathrm{E}-5$ & 2 \\
\hline 3691 & 1.0 & EPI_ISL_420577 & 7.488399999999989E-4 & $3.874 \mathrm{E}-5$ & 2 \\
\hline 3726 & 1.0 & EPI_ISL_424943 & 8.094999999999991E-4 & $7.534000000000013 \mathrm{E}-5$ & 6 \\
\hline 3726 & 1.0 & EPI_ISL_422503 & 7.767199999999991E-4 & $7.534000000000013 \mathrm{E}-5$ & 6 \\
\hline 3726 & 1.0 & EPI_ISL_427585 & 7.44479999999999E-4 & $7.534000000000013 \mathrm{E}-5$ & 6 \\
\hline 3726 & 1.0 & EPI_ISL_427631 & 7.788599999999991E-4 & $7.534000000000013 \mathrm{E}-5$ & 6 \\
\hline 3726 & 1.0 & EPI_ISL_422510 & 7.746599999999991E-4 & $7.534000000000013 \mathrm{E}-5$ & 6 \\
\hline 3726 & 1.0 & EPI_ISL_427588 & 7.44479999999999E-4 & $7.534000000000013 \mathrm{E}-5$ & 6 \\
\hline 3739 & 1.0 & EPI_ISL_424935 & 7.313999999999992E-4 & 4.359999999999911E-6 & 2 \\
\hline 3739 & 1.0 & EPI_ISL_418190 & 7.313999999999992E-4 & 4.359999999999911E-6 & 2 \\
\hline 3742 & 1.0 & EPI_ISL_427629 & 8.007999999999993E-4 & 4.0929999999999786E-5 & 6 \\
\hline 3742 & 1.0 & EPI_ISL_427553 & 7.707699999999992E-4 & 4.0929999999999786E-5 & 6 \\
\hline
\end{tabular}


Table S4: Clusters identified using a genetic distance threshold within $1 \%$ of the distribution of patristic distances within the entire tree. The minimum percentile threshold that maximized the number of clusters was chosen as the optimal threshold by performing multiple clustering runs on randomly sampled patristic distance distributions (1 million for each run) in Phylopart v2 (46).

\begin{tabular}{|c|c|c|c|c|c|}
\hline clustername & bootstrap & leafname & branchPath & medianOfDistances & sequencesperCluster \\
\hline 3742 & 1.0 & EPI_ISL_421422 & 7.707699999999992E-4 & "4.0929999999999786E-5 & $\overline{6} 6$ \\
\hline 3742 & 1.0 & EPI_ISL_421388 & 7.707699999999992E-4 & $4.0929999999999786 \mathrm{E}-5$ & 6 \\
\hline 3742 & 1.0 & EPI_ISL_427514 & 8.029799999999993E-4 & $4.0929999999999786 \mathrm{E}-5$ & 6 \\
\hline 3742 & 1.0 & EPI_ISL_421389 & 7.707699999999992E-4 & $4.0929999999999786 \mathrm{E}-5$ & 6 \\
\hline 3791 & 1.0 & EPI_ISL_420574 & 7.578899999999995E-4 & 3.956999999999984E-5 & 2 \\
\hline 3791 & 1.0 & EPI_ISL_421581 & 7.226799999999994E-4 & 3.956999999999984E-5 & 2 \\
\hline 3801 & 1.0 & EPI_ISL_418973 & 7.636099999999993E-4 & 4.310999999999985E-5 & 10 \\
\hline 3801 & 1.0 & EPI_ISL_421583 & 7.313999999999992E-4 & 4.310999999999985E-5 & 10 \\
\hline 3801 & 1.0 & EPI_ISL_420581 & 7.313999999999992E-4 & 4.310999999999985E-5 & 10 \\
\hline 3801 & 1.0 & EPI_ISL_426618 & 7.614299999999994E-4 & 4.310999999999985E-5 & 10 \\
\hline 3801 & 1.0 & EPI_ISL_420297 & 7.313999999999992E-4 & 4.310999999999985E-5 & 10 \\
\hline 3801 & 1.0 & EPI_ISL_428800 & 7.964099999999996E-4 & 4.310999999999985E-5 & 10 \\
\hline 3801 & 1.0 & EPI_ISL_422512 & 7.248599999999994E-4 & 4.310999999999985E-5 & 10 \\
\hline 3801 & 1.0 & EPI_ISL_421421 & 7.313999999999992E-4 & 4.310999999999985E-5 & 10 \\
\hline 3801 & 1.0 & EPI_ISL_424953 & 7.570699999999994E-4 & 4.310999999999985E-5 & 10 \\
\hline 3801 & 1.0 & EPI_ISL_421597 & 7.313999999999992E-4 & 4.310999999999985E-5 & 10 \\
\hline 3894 & 1.0 & EPI_ISL_427064 & 7.843599999999997E-4 & $3.910000000000003 \mathrm{E}-5$ & 2 \\
\hline 3894 & 1.0 & EPI_ISL_427065 & 7.496199999999997E-4 & $3.910000000000003 \mathrm{E}-5$ & 2 \\
\hline 3915 & 1.0 & EPI_ISL_422906 & 7.519599999999995E-4 & 3.962999999999992E-5 & 2 \\
\hline 3915 & 1.0 & EPI_ISL_422703 & 7.166899999999995E-4 & 3.962999999999992E-5 & 2 \\
\hline 3930 & 0.944 & EPI_ISL_415485 & 7.559599999999995E-4 & $3.874 \mathrm{E}-5$ & 3 \\
\hline 3930 & 0.944 & EPI_ISL_415474 & 7.215799999999994E-4 & $3.874 \mathrm{E}-5$ & 3 \\
\hline 3930 & 0.944 & EPI_ISL_415473 & 7.215799999999994E-4 & $3.874 \mathrm{E}-5$ & 3 \\
\hline 3936 & 1.0 & EPI_ISL_427501 & $6.307299999999997 \mathrm{E}-4$ & $7.390000000000001 \mathrm{E}-5$ & 2 \\
\hline 3936 & 1.0 & EPI_ISL_424941 & 6.299499999999998E-4 & $7.390000000000001 \mathrm{E}-5$ & 2 \\
\hline 3939 & 1.0 & EPI_ISL_426939 & 7.040999999999997E-4 & 3.987999999999995E-5 & 2 \\
\hline
\end{tabular}


Table S4: Clusters identified using a genetic distance threshold within $1 \%$ of the distribution of patristic distances within the entire tree. The minimum percentile threshold that maximized the number of clusters was chosen as the optimal threshold by performing multiple clustering runs on randomly sampled patristic distance distributions (1 million for each run) in Phylopart v2 (46).

\begin{tabular}{|c|c|c|c|c|c|}
\hline clustername & bootstrap & leafname & branchPath & medianOfDistances & sequencesperCluster \\
\hline 3939 & 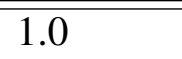 & "EPI_ISL_426657 & 6.685799999999997E-4 & 3.987999999999995E-5 & 2 \\
\hline 3956 & 1.0 & EPI_ISL_421346 & 5.977499999999996E-4 & 4.359999999999911E-6 & 6 \\
\hline 3956 & 1.0 & EPI_ISL_421545 & 5.977499999999996E-4 & 4.359999999999911E-6 & 6 \\
\hline 3956 & 1.0 & EPI_ISL_421544 & 5.977499999999996E-4 & 4.359999999999911E-6 & 6 \\
\hline 3956 & 1.0 & EPI_ISL_421347 & 6.308899999999997E-4 & 4.359999999999911E-6 & 6 \\
\hline 3956 & 1.0 & EPI_ISL_421543 & 5.977499999999996E-4 & 4.359999999999911E-6 & 6 \\
\hline 3956 & 1.0 & EPI_ISL_421553 & $5.977499999999996 \mathrm{E}-4$ & 4.359999999999911E-6 & 6 \\
\hline 3967 & 1.0 & EPI_ISL_429517 & 6.605999999999999E-4 & 7.312000000000009E-5 & 2 \\
\hline 3967 & 1.0 & EPI_ISL_429585 & 6.605999999999999E-4 & 7.312000000000009E-5 & 2 \\
\hline 3988 & 1.0 & EPI_ISL_420761 & 6.608199999999999E-4 & 3.895999999999999E-5 & 2 \\
\hline 3988 & 1.0 & EPI_ISL_422408 & 6.262199999999998E-4 & 3.895999999999999E-5 & 2 \\
\hline 3995 & 1.0 & EPI_ISL_429067 & 5.912199999999998E-4 & 4.359999999999911E-6 & 2 \\
\hline 3995 & 1.0 & EPI_ISL_429989 & 5.912199999999998E-4 & 4.359999999999911E-6 & 2 \\
\hline 4003 & 1.0 & EPI_ISL_420079 & 0.00103186 & $5.297999999999978 \mathrm{E}-5$ & 2 \\
\hline 4003 & 1.0 & EPI_ISL_420071 & $9.8324 \mathrm{E}-4$ & $5.297999999999978 \mathrm{E}-5$ & 2 \\
\hline 4056 & 1.0 & EPI_ISL_429403 & 6.366399999999996E-4 & 6.539999999999758E-6 & 3 \\
\hline 4056 & 1.0 & EPI_ISL_429267 & 6.388199999999996E-4 & $6.539999999999758 \mathrm{E}-6$ & 3 \\
\hline 4056 & 1.0 & EPI_ISL_429265 & 6.388199999999996E-4 & 6.539999999999758E-6 & 3 \\
\hline 4061 & 1.0 & EPI_ISL_429361 & 5.999399999999997E-4 & 4.359999999999911E-6 & 2 \\
\hline 4061 & 1.0 & EPI_ISL_429416 & 5.999399999999997E-4 & 4.359999999999911E-6 & 2 \\
\hline 4064 & 1.0 & EPI_ISL_427639 & 6.321399999999998E-4 & $3.874 \mathrm{E}-5$ & 2 \\
\hline 4064 & 1.0 & EPI_ISL_429441 & 5.977599999999997E-4 & $3.874 \mathrm{E}-5$ & 2 \\
\hline 4108 & 1.0 & EPI_ISL_424335 & 5.955799999999997E-4 & 4.0919999999999845E-5 & 3 \\
\hline 4108 & 1.0 & EPI_ISL_429794 & 6.321399999999998E-4 & 4.0919999999999845E-5 & 3 \\
\hline 4108 & 1.0 & EPI_ISL_429722 & 6.321399999999998E-4 & 4.0919999999999845E-5 & 3 \\
\hline 4115 & 1.0 & EPI_ISL_427250 & 6.299599999999997E-4 & 4.0919999999999845E-5 & 6 \\
\hline
\end{tabular}


Table S4: Clusters identified using a genetic distance threshold within $1 \%$ of the distribution of patristic distances within the entire tree. The minimum percentile threshold that maximized the number of clusters was chosen as the optimal threshold by performing multiple clustering runs on randomly sampled patristic distance distributions (1 million for each run) in Phylopart v2 (46).

\begin{tabular}{|c|c|c|c|c|c|}
\hline clustername & bootstrap & leafname & branchPath & medianOfDistances & sequencesperCluster \\
\hline 4115 & 1.0 & "EPI_ISL_429290 & 6.455199999999998E-4 & "4.0919999999999845E-5 & 6 \\
\hline 4115 & 1.0 & EPI_ISL_426687 & 5.977599999999997E-4 & 4.0919999999999845E-5 & 6 \\
\hline 4115 & 1.0 & EPI_ISL_429275 & 5.977599999999997E-4 & 4.0919999999999845E-5 & 6 \\
\hline 4115 & 1.0 & EPI_ISL_429382 & 5.977599999999997E-4 & 4.0919999999999845E-5 & 6 \\
\hline 4115 & 1.0 & EPI_ISL_429274 & 5.977599999999997E-4 & 4.0919999999999845E-5 & 6 \\
\hline 4129 & 1.0 & EPI_ISL_422508 & 6.693399999999997E-4 & $3.875999999999988 \mathrm{E}-5$ & 2 \\
\hline 4129 & 1.0 & EPI_ISL_421590 & 6.349399999999996E-4 & 3.875999999999988E-5 & 2 \\
\hline 4136 & 1.0 & EPI_ISL_424491 & 6.349399999999996E-4 & 4.359999999999911E-6 & 2 \\
\hline 4136 & 1.0 & EPI_ISL_424409 & 6.349399999999996E-4 & 4.359999999999911E-6 & 2 \\
\hline 4142 & 1.0 & EPI_ISL_424314 & 6.517599999999994E-4 & 8.430999999999868E-5 & 185 \\
\hline 4142 & 1.0 & EPI_ISL_427196 & 6.282799999999991E-4 & 8.430999999999868E-5 & 185 \\
\hline 4142 & 1.0 & EPI_ISL_418879 & 7.438999999999997E-4 & 8.430999999999868E-5 & 185 \\
\hline 4142 & 1.0 & EPI_ISL_426093 & 6.604799999999992E-4 & 8.430999999999868E-5 & 185 \\
\hline 4142 & 1.0 & EPI_ISL_426079 & 6.324799999999991E-4 & 8.430999999999868E-5 & 185 \\
\hline 4142 & 1.0 & EPI_ISL_418876 & 6.439299999999988E-4 & 8.430999999999868E-5 & 185 \\
\hline 4142 & 1.0 & EPI_ISL_424193 & 6.413599999999988E-4 & 8.430999999999868E-5 & 185 \\
\hline 4142 & 1.0 & EPI_ISL_427256 & 6.517599999999994E-4 & 8.430999999999868E-5 & 185 \\
\hline 4142 & 1.0 & EPI_ISL_424277 & 8.507399999999987E-4 & 8.430999999999868E-5 & 185 \\
\hline 4142 & 1.0 & EPI_ISL_422967 & $6.413599999999988 \mathrm{E}-4$ & 8.430999999999868E-5 & 185 \\
\hline 4142 & 1.0 & EPI_ISL_422998 & $6.217399999999992 \mathrm{E}-4$ & 8.430999999999868E-5 & 185 \\
\hline 4142 & 1.0 & EPI_ISL_427181 & 6.861399999999995E-4 & 8.430999999999868E-5 & 185 \\
\hline 4142 & 1.0 & EPI_ISL_418904 & 6.604799999999992E-4 & 8.430999999999868E-5 & 185 \\
\hline 4142 & 1.0 & EPI_ISL_426080 & 6.348199999999989E-4 & 8.430999999999868E-5 & 185 \\
\hline 4142 & 1.0 & EPI_ISL_429615 & 6.368399999999996E-4 & 8.430999999999868E-5 & 185 \\
\hline 4142 & 1.0 & EPI_ISL_418900 & 6.435399999999988E-4 & 8.430999999999868E-5 & 185 \\
\hline 4142 & 1.0 & EPI_ISL_424331 & 6.473999999999995E-4 & 8.430999999999868E-5 & 185 \\
\hline
\end{tabular}


Table S4: Clusters identified using a genetic distance threshold within $1 \%$ of the distribution of patristic distances within the entire tree. The minimum percentile threshold that maximized the number of clusters was chosen as the optimal threshold by performing multiple clustering runs on randomly sampled patristic distance distributions (1 million for each run) in Phylopart v2 (46).

\begin{tabular}{|c|c|c|c|c|c|}
\hline clustername & bootstrap & leafname & branchPath & medianOfDistances & sequencesperCluster \\
\hline 4142 & 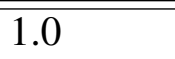 & "EPI_ISL_423024 & 6.282799999999991E-4 & 8.430999999999868E-5 & 185 \\
\hline 4142 & 1.0 & EPI_ISL_429653 & 6.30459999999999E-4 & 8.430999999999868E-5 & 185 \\
\hline 4142 & 1.0 & EPI_ISL_429359 & 7.207199999999996E-4 & 8.430999999999868E-5 & 185 \\
\hline 4142 & 1.0 & EPI_ISL_427254 & 6.517599999999994E-4 & 8.430999999999868E-5 & 185 \\
\hline 4142 & 1.0 & EPI_ISL_424242 & 7.342199999999992E-4 & 8.430999999999868E-5 & 185 \\
\hline 4142 & 1.0 & EPI_ISL_427200 & 6.582999999999993E-4 & 8.430999999999868E-5 & 185 \\
\hline 4142 & 1.0 & EPI_ISL_426091 & 6.413599999999988E-4 & 8.430999999999868E-5 & 185 \\
\hline 4142 & 1.0 & EPI_ISL_422979 & 6.427499999999988E-4 & 8.430999999999868E-5 & 185 \\
\hline 4142 & 1.0 & EPI_ISL_424205 & 6.609799999999984E-4 & 8.430999999999868E-5 & 185 \\
\hline 4142 & 1.0 & EPI_ISL_427202 & 6.539399999999993E-4 & 8.430999999999868E-5 & 185 \\
\hline 4142 & 1.0 & EPI_ISL_426087 & 6.738299999999989E-4 & 8.430999999999868E-5 & 185 \\
\hline 4142 & 1.0 & EPI_ISL_417454 & 6.71379999999999E-4 & 8.430999999999868E-5 & 185 \\
\hline 4142 & 1.0 & EPI_ISL_429631 & 6.413599999999988E-4 & 8.430999999999868E-5 & 185 \\
\hline 4142 & 1.0 & EPI_ISL_417451 & 6.32639999999999E-4 & 8.430999999999868E-5 & 185 \\
\hline 4142 & 1.0 & EPI_ISL_427209 & 6.839699999999995E-4 & 8.430999999999868E-5 & 185 \\
\hline 4142 & 1.0 & EPI_ISL_424200 & 6.32639999999999E-4 & 8.430999999999868E-5 & 185 \\
\hline 4142 & 1.0 & EPI_ISL_416647 & 6.369999999999989E-4 & 8.430999999999868E-5 & 185 \\
\hline 4142 & 1.0 & EPI_ISL_429608 & 6.299599999999998E-4 & 8.430999999999868E-5 & 185 \\
\hline 4142 & 1.0 & EPI_ISL_427215 & 6.604799999999992E-4 & 8.430999999999868E-5 & 185 \\
\hline 4142 & 1.0 & EPI_ISL_418929 & 6.32639999999999E-4 & 8.430999999999868E-5 & 185 \\
\hline 4142 & 1.0 & EPI_ISL_427230 & 6.861799999999995E-4 & 8.430999999999868E-5 & 185 \\
\hline 4142 & 1.0 & EPI_ISL_418924 & 6.413599999999988E-4 & 8.430999999999868E-5 & 185 \\
\hline 4142 & 1.0 & EPI_ISL_427255 & 6.517599999999994E-4 & 8.430999999999868E-5 & 185 \\
\hline 4142 & 1.0 & EPI_ISL_426081 & 6.71379999999999E-4 & 8.430999999999868E-5 & 185 \\
\hline 4142 & 1.0 & EPI_ISL_422978 & 6.239199999999992E-4 & 8.430999999999868E-5 & 185 \\
\hline 4142 & 1.0 & EPI_ISL_418923 & 6.369999999999989E-4 & 8.430999999999868E-5 & 185 \\
\hline
\end{tabular}


Table S4: Clusters identified using a genetic distance threshold within $1 \%$ of the distribution of patristic distances within the entire tree. The minimum percentile threshold that maximized the number of clusters was chosen as the optimal threshold by performing multiple clustering runs on randomly sampled patristic distance distributions (1 million for each run) in Phylopart v2 (46).

\begin{tabular}{|c|c|c|c|c|c|}
\hline clustername & bootstrap & leafname & branchPath & medianOfDistances & sequencesperCluster \\
\hline 4142 & 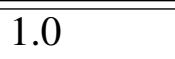 & "EPI_ISL_424333 & 6.343299999999997E-4 & 8.430999999999868E-5 & 185 \\
\hline 4142 & 1.0 & EPI_ISL_416708 & $6.282799999999991 \mathrm{E}-4$ & 8.430999999999868E-5 & 185 \\
\hline 4142 & 1.0 & EPI_ISL_418932 & 6.670199999999991E-4 & 8.430999999999868E-5 & 185 \\
\hline 4142 & 1.0 & EPI_ISL_424202 & 6.413599999999988E-4 & 8.430999999999868E-5 & 185 \\
\hline 4142 & 1.0 & EPI_ISL_416691 & 6.626599999999992E-4 & 8.430999999999868E-5 & 185 \\
\hline 4142 & 1.0 & EPI_ISL_416719 & 6.413599999999988E-4 & 8.430999999999868E-5 & 185 \\
\hline 4142 & 1.0 & EPI_ISL_424204 & 6.604799999999992E-4 & 8.430999999999868E-5 & 185 \\
\hline 4142 & 1.0 & EPI_ISL_424309 & 6.71379999999999E-4 & 8.430999999999868E-5 & 185 \\
\hline 4142 & 1.0 & EPI_ISL_429607 & 9.494899999999994E-4 & 8.430999999999868E-5 & 185 \\
\hline 4142 & 1.0 & EPI_ISL_426104 & 6.260999999999991E-4 & 8.430999999999868E-5 & 185 \\
\hline 4142 & 1.0 & EPI_ISL_424310 & 6.173799999999993E-4 & 8.430999999999868E-5 & 185 \\
\hline 4142 & 1.0 & EPI_ISL_424227 & 6.495799999999994E-4 & 8.430999999999868E-5 & 185 \\
\hline 4142 & 1.0 & EPI_ISL_427189 & 6.604799999999992E-4 & 8.430999999999868E-5 & 185 \\
\hline 4142 & 1.0 & EPI_ISL_427191 & 6.413599999999988E-4 & 8.430999999999868E-5 & 185 \\
\hline 4142 & 1.0 & EPI_ISL_427210 & 7.363999999999991E-4 & 8.430999999999868E-5 & 185 \\
\hline 4142 & 1.0 & EPI_ISL_422983 & 7.335299999999992E-4 & 8.430999999999868E-5 & 185 \\
\hline 4142 & 1.0 & EPI_ISL_424221 & 6.500799999999986E-4 & 8.430999999999868E-5 & 185 \\
\hline 4142 & 1.0 & EPI_ISL_429600 & 6.67869999999999E-4 & 8.430999999999868E-5 & 185 \\
\hline 4142 & 1.0 & EPI_ISL_418913 & 6.042999999999996E-4 & 8.430999999999868E-5 & 185 \\
\hline 4142 & 1.0 & EPI_ISL_424203 & 6.604799999999992E-4 & 8.430999999999868E-5 & 185 \\
\hline 4142 & 1.0 & EPI_ISL_417342 & 6.413599999999988E-4 & 8.430999999999868E-5 & 185 \\
\hline 4142 & 1.0 & EPI_ISL_424178 & 6.745299999999989E-4 & 8.430999999999868E-5 & 185 \\
\hline 4142 & 1.0 & EPI_ISL_424302 & 6.413599999999988E-4 & 8.430999999999868E-5 & 185 \\
\hline 4142 & 1.0 & EPI_ISL_427239 & 7.599099999999995E-4 & 8.430999999999868E-5 & 185 \\
\hline 4142 & 1.0 & EPI_ISL_424238 & 6.72479999999999E-4 & 8.430999999999868E-5 & 185 \\
\hline 4142 & 1.0 & EPI_ISL_422985 & 6.32639999999999E-4 & 8.430999999999868E-5 & 185 \\
\hline
\end{tabular}


Table S4: Clusters identified using a genetic distance threshold within $1 \%$ of the distribution of patristic distances within the entire tree. The minimum percentile threshold that maximized the number of clusters was chosen as the optimal threshold by performing multiple clustering runs on randomly sampled patristic distance distributions (1 million for each run) in Phylopart v2 (46).

\begin{tabular}{|c|c|c|c|c|c|}
\hline clustername & bootstrap & leafname & branchPath & medianOfDistances & sequencesperCluster \\
\hline 4142 & 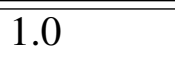 & "EPI_ISL_427236 & 6.883999999999995E-4 & 8.430999999999868E-5 & 185 \\
\hline 4142 & 1.0 & EPI_ISL_416707 & 6.348199999999989E-4 & 8.430999999999868E-5 & 185 \\
\hline 4142 & 1.0 & EPI_ISL_424305 & 6.631599999999984E-4 & 8.430999999999868E-5 & 185 \\
\hline 4142 & 1.0 & EPI_ISL_418878 & 7.78819999999999E-4 & 8.430999999999868E-5 & 185 \\
\hline 4142 & 1.0 & EPI_ISL_424208 & 6.364999999999997E-4 & 8.430999999999868E-5 & 185 \\
\hline 4142 & 1.0 & EPI_ISL_424312 & 6.413599999999988E-4 & 8.430999999999868E-5 & 185 \\
\hline 4142 & 1.0 & EPI_ISL_427225 & 7.579399999999995E-4 & 8.430999999999868E-5 & 185 \\
\hline 4142 & 1.0 & EPI_ISL_416693 & 6.648399999999991E-4 & 8.430999999999868E-5 & 185 \\
\hline 4142 & 1.0 & EPI_ISL_416653 & 6.631599999999984E-4 & 8.430999999999868E-5 & 185 \\
\hline 4142 & 1.0 & EPI_ISL_426116 & 6.32639999999999E-4 & 8.430999999999868E-5 & 185 \\
\hline 4142 & 1.0 & EPI_ISL_423001 & 6.69199999999999E-4 & 8.430999999999868E-5 & 185 \\
\hline 4142 & 1.0 & EPI_ISL_426115 & 7.705699999999992E-4 & 8.430999999999868E-5 & 185 \\
\hline 4142 & 1.0 & EPI_ISL_429622 & 6.648399999999991E-4 & 8.430999999999868E-5 & 185 \\
\hline 4142 & 1.0 & EPI_ISL_424245 & 6.348199999999989E-4 & 8.430999999999868E-5 & 185 \\
\hline 4142 & 1.0 & EPI_ISL_429617 & 6.71379999999999E-4 & 8.430999999999868E-5 & 185 \\
\hline 4142 & 1.0 & EPI_ISL_427194 & 6.495799999999994E-4 & 8.430999999999868E-5 & 185 \\
\hline 4142 & 1.0 & EPI_ISL_424324 & 6.696599999999991E-4 & 8.430999999999868E-5 & 185 \\
\hline 4142 & 1.0 & EPI_ISL_427247 & 6.604799999999992E-4 & 8.430999999999868E-5 & 185 \\
\hline 4142 & 1.0 & EPI_ISL_424259 & 6.413599999999988E-4 & 8.430999999999868E-5 & 185 \\
\hline 4142 & 1.0 & EPI_ISL_426134 & 6.430499999999995E-4 & 8.430999999999868E-5 & 185 \\
\hline 4142 & 1.0 & EPI_ISL_424246 & 6.435399999999988E-4 & 8.430999999999868E-5 & 185 \\
\hline 4142 & 1.0 & EPI_ISL_429654 & 6.517599999999994E-4 & 8.430999999999868E-5 & 185 \\
\hline 4142 & 1.0 & EPI_ISL_423018 & 6.413599999999988E-4 & 8.430999999999868E-5 & 185 \\
\hline 4142 & 1.0 & EPI_ISL_424229 & 6.635499999999991E-4 & 8.430999999999868E-5 & 185 \\
\hline 4142 & 1.0 & EPI_ISL_424212 & 6.386799999999997E-4 & 8.430999999999868E-5 & 185 \\
\hline 4142 & 1.0 & EPI_ISL_429651 & 6.413599999999988E-4 & 8.430999999999868E-5 & 185 \\
\hline
\end{tabular}


Table S4: Clusters identified using a genetic distance threshold within $1 \%$ of the distribution of patristic distances within the entire tree. The minimum percentile threshold that maximized the number of clusters was chosen as the optimal threshold by performing multiple clustering runs on randomly sampled patristic distance distributions (1 million for each run) in Phylopart v2 (46).

\begin{tabular}{|c|c|c|c|c|c|}
\hline clustername & bootstrap & leafname & branchPath & medianOfDistances & sequencesperCluster \\
\hline 4142 & 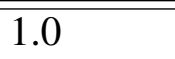 & "EPI_ISL_422970 & 6.30459999999999E-4 & 8.430999999999868E-5 & 185 \\
\hline 4142 & 1.0 & EPI_ISL_429652 & 6.380799999999991E-4 & 8.430999999999868E-5 & 185 \\
\hline 4142 & 1.0 & EPI_ISL_424256 & 6.824099999999995E-4 & 8.430999999999868E-5 & 185 \\
\hline 4142 & 1.0 & EPI_ISL_424340 & 6.413599999999988E-4 & 8.430999999999868E-5 & 185 \\
\hline 4142 & 1.0 & EPI_ISL_424214 & 6.926399999999993E-4 & 8.430999999999868E-5 & 185 \\
\hline 4142 & 1.0 & EPI_ISL_422976 & $6.413599999999988 \mathrm{E}-4$ & 8.430999999999868E-5 & 185 \\
\hline 4142 & 1.0 & EPI_ISL_429614 & 6.71399999999999E-4 & 8.430999999999868E-5 & 185 \\
\hline 4142 & 1.0 & EPI_ISL_416438 & 6.413599999999988E-4 & 8.430999999999868E-5 & 185 \\
\hline 4142 & 1.0 & EPI_ISL_427260 & 6.413599999999988E-4 & 8.430999999999868E-5 & 185 \\
\hline 4142 & 1.0 & EPI_ISL_427207 & 6.457199999999987E-4 & 8.430999999999868E-5 & 185 \\
\hline 4142 & 1.0 & EPI_ISL_418054 & 6.604799999999992E-4 & 8.430999999999868E-5 & 185 \\
\hline 4142 & 1.0 & EPI_ISL_424235 & 7.024199999999991E-4 & 8.430999999999868E-5 & 185 \\
\hline 4142 & 1.0 & EPI_ISL_427180 & 6.30459999999999E-4 & 8.430999999999868E-5 & 185 \\
\hline 4142 & 1.0 & EPI_ISL_429637 & 6.582999999999993E-4 & 8.430999999999868E-5 & 185 \\
\hline 4142 & 1.0 & EPI_ISL_417382 & 6.32639999999999E-4 & 8.430999999999868E-5 & 185 \\
\hline 4142 & 1.0 & EPI_ISL_427216 & 6.413599999999988E-4 & 8.430999999999868E-5 & 185 \\
\hline 4142 & 1.0 & EPI_ISL_418951 & $6.413599999999988 \mathrm{E}-4$ & 8.430999999999868E-5 & 185 \\
\hline 4142 & 1.0 & EPI_ISL_427222 & 6.474099999999994E-4 & 8.430999999999868E-5 & 185 \\
\hline 4142 & 1.0 & EPI_ISL_424270 & 6.817899999999995E-4 & 8.430999999999868E-5 & 185 \\
\hline 4142 & 1.0 & EPI_ISL_427199 & 6.413599999999988E-4 & 8.430999999999868E-5 & 185 \\
\hline 4142 & 1.0 & EPI_ISL_423010 & 6.413599999999988E-4 & 8.430999999999868E-5 & 185 \\
\hline 4142 & 1.0 & EPI_ISL_416698 & $6.260999999999991 \mathrm{E}-4$ & 8.430999999999868E-5 & 185 \\
\hline 4142 & 1.0 & EPI_ISL_429655 & 6.779299999999988E-4 & 8.430999999999868E-5 & 185 \\
\hline 4142 & 1.0 & EPI_ISL_417455 & 6.217399999999992E-4 & 8.430999999999868E-5 & 185 \\
\hline 4142 & 1.0 & EPI_ISL_427228 & 6.282799999999991E-4 & 8.430999999999868E-5 & 185 \\
\hline 4142 & 1.0 & EPI_ISL_427234 & 9.075999999999995E-4 & 8.430999999999868E-5 & 185 \\
\hline
\end{tabular}


Table S4: Clusters identified using a genetic distance threshold within $1 \%$ of the distribution of patristic distances within the entire tree. The minimum percentile threshold that maximized the number of clusters was chosen as the optimal threshold by performing multiple clustering runs on randomly sampled patristic distance distributions (1 million for each run) in Phylopart v2 (46).

\begin{tabular}{|c|c|c|c|c|c|}
\hline clustername & bootstrap & leafname & branchPath & medianOfDistances & sequencesperCluster \\
\hline 4142 & 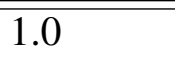 & "EPI_ISL_418032 & 6.544399999999985E-4 & 8.430999999999868E-5 & 185 \\
\hline 4142 & 1.0 & EPI_ISL_422981 & 6.413599999999988E-4 & 8.430999999999868E-5 & 185 \\
\hline 4142 & 1.0 & EPI_ISL_424337 & 6.71379999999999E-4 & 8.430999999999868E-5 & 185 \\
\hline 4142 & 1.0 & EPI_ISL_427197 & 6.413599999999988E-4 & 8.430999999999868E-5 & 185 \\
\hline 4142 & 1.0 & EPI_ISL_424189 & 6.566199999999985E-4 & 8.430999999999868E-5 & 185 \\
\hline 4142 & 1.0 & EPI_ISL_424197 & 7.042599999999991E-4 & 8.430999999999868E-5 & 185 \\
\hline 4142 & 1.0 & EPI_ISL_422969 & 6.239199999999992E-4 & 8.430999999999868E-5 & 185 \\
\hline 4142 & 1.0 & EPI_ISL_416704 & 6.611999999999992E-4 & 8.430999999999868E-5 & 185 \\
\hline 4142 & 1.0 & EPI_ISL_426125 & 7.766899999999989E-4 & 8.430999999999868E-5 & 185 \\
\hline 4142 & 1.0 & EPI_ISL_418068 & 8.666199999999987E-4 & 8.430999999999868E-5 & 185 \\
\hline 4142 & 1.0 & EPI_ISL_424184 & 6.413599999999988E-4 & 8.430999999999868E-5 & 185 \\
\hline 4142 & 1.0 & EPI_ISL_418067 & 6.30459999999999E-4 & 8.430999999999868E-5 & 185 \\
\hline 4142 & 1.0 & EPI_ISL_416434 & 6.670199999999991E-4 & 8.430999999999868E-5 & 185 \\
\hline 4142 & 1.0 & EPI_ISL_418053 & 6.413599999999988E-4 & 8.430999999999868E-5 & 185 \\
\hline 4142 & 1.0 & EPI_ISL_429602 & 6.604899999999991E-4 & 8.430999999999868E-5 & 185 \\
\hline 4142 & 1.0 & EPI_ISL_416452 & 6.343599999999997E-4 & 8.430999999999868E-5 & 185 \\
\hline 4142 & 1.0 & EPI_ISL_423031 & 6.304699999999991E-4 & 8.430999999999868E-5 & 185 \\
\hline 4142 & 1.0 & EPI_ISL_418933 & 6.413599999999988E-4 & 8.430999999999868E-5 & 185 \\
\hline 4142 & 1.0 & EPI_ISL_424251 & 6.413599999999988E-4 & 8.430999999999868E-5 & 185 \\
\hline 4142 & 1.0 & EPI_ISL_426103 & 6.369999999999989E-4 & 8.430999999999868E-5 & 185 \\
\hline 4142 & 1.0 & EPI_ISL_426112 & 6.413599999999988E-4 & 8.430999999999868E-5 & 185 \\
\hline 4142 & 1.0 & EPI_ISL_418081 & 6.604799999999992E-4 & 8.430999999999868E-5 & 185 \\
\hline 4142 & 1.0 & EPI_ISL_418880 & 6.478999999999987E-4 & 8.430999999999868E-5 & 185 \\
\hline 4142 & 1.0 & EPI_ISL_424190 & 6.369999999999989E-4 & 8.430999999999868E-5 & 185 \\
\hline 4142 & 1.0 & EPI_ISL_416716 & 6.413599999999988E-4 & 8.430999999999868E-5 & 185 \\
\hline 4142 & 1.0 & EPI_ISL_426515 & 6.670199999999991E-4 & 8.430999999999868E-5 & 185 \\
\hline
\end{tabular}


Table S4: Clusters identified using a genetic distance threshold within $1 \%$ of the distribution of patristic distances within the entire tree. The minimum percentile threshold that maximized the number of clusters was chosen as the optimal threshold by performing multiple clustering runs on randomly sampled patristic distance distributions (1 million for each run) in Phylopart v2 (46).

\begin{tabular}{|c|c|c|c|c|c|}
\hline clustername & bootstrap & leafname & branchPath & medianOfDistances & sequencesperCluster \\
\hline 4142 & 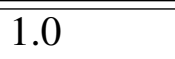 & "EPI_ISL_417341 & 6.260999999999991E-4 & 8.430999999999868E-5 & 185 \\
\hline 4142 & 1.0 & EPI_ISL_415625 & 6.587999999999984E-4 & 8.430999999999868E-5 & 185 \\
\hline 4142 & 1.0 & EPI_ISL_427246 & 6.670199999999991E-4 & 8.430999999999868E-5 & 185 \\
\hline 4142 & 1.0 & EPI_ISL_424211 & 6.30459999999999E-4 & 8.430999999999868E-5 & 185 \\
\hline 4142 & 1.0 & EPI_ISL_429635 & 6.495799999999994E-4 & 8.430999999999868E-5 & 185 \\
\hline 4142 & 1.0 & EPI_ISL_426113 & 6.583099999999992E-4 & 8.430999999999868E-5 & 185 \\
\hline 4142 & 1.0 & EPI_ISL_418875 & 6.32639999999999E-4 & 8.430999999999868E-5 & 185 \\
\hline 4142 & 1.0 & EPI_ISL_424316 & 6.517599999999994E-4 & 8.430999999999868E-5 & 185 \\
\hline 4142 & 1.0 & EPI_ISL_416699 & 6.130199999999994E-4 & 8.430999999999868E-5 & 185 \\
\hline 4142 & 1.0 & EPI_ISL_414616 & 6.391799999999989E-4 & 8.430999999999868E-5 & 185 \\
\hline 4142 & 1.0 & EPI_ISL_426135 & 6.413599999999988E-4 & 8.430999999999868E-5 & 185 \\
\hline 4142 & 1.0 & EPI_ISL_418894 & 6.108399999999994E-4 & 8.430999999999868E-5 & 185 \\
\hline 4142 & 1.0 & EPI_ISL_427205 & 6.413599999999988E-4 & 8.430999999999868E-5 & 185 \\
\hline 4142 & 1.0 & EPI_ISL_427253 & 6.861499999999995E-4 & 8.430999999999868E-5 & 185 \\
\hline 4142 & 1.0 & EPI_ISL_422977 & 6.517599999999994E-4 & 8.430999999999868E-5 & 185 \\
\hline 4142 & 1.0 & EPI_ISL_429613 & 6.32639999999999E-4 & 8.430999999999868E-5 & 185 \\
\hline 4142 & 1.0 & EPI_ISL_427241 & 6.408599999999996E-4 & 8.430999999999868E-5 & 185 \\
\hline 4142 & 1.0 & EPI_ISL_424183 & 6.413599999999988E-4 & 8.430999999999868E-5 & 185 \\
\hline 4142 & 1.0 & EPI_ISL_429597 & 6.384699999999994E-4 & 8.430999999999868E-5 & 185 \\
\hline 4142 & 1.0 & EPI_ISL_424313 & 6.413599999999988E-4 & 8.430999999999868E-5 & 185 \\
\hline 4142 & 1.0 & EPI_ISL_415597 & 6.413599999999988E-4 & 8.430999999999868E-5 & 185 \\
\hline 4142 & 1.0 & EPI_ISL_427188 & 6.604799999999992E-4 & 8.430999999999868E-5 & 185 \\
\hline 4142 & 1.0 & EPI_ISL_423032 & 6.992199999999992E-4 & 8.430999999999868E-5 & 185 \\
\hline 4142 & 1.0 & EPI_ISL_427224 & 8.001599999999994E-4 & 8.430999999999868E-5 & 185 \\
\hline 4142 & 1.0 & EPI_ISL_416724 & 6.260999999999991E-4 & 8.430999999999868E-5 & 185 \\
\hline 4142 & 1.0 & EPI_ISL_427242 & 6.861699999999994E-4 & 8.430999999999868E-5 & 185 \\
\hline
\end{tabular}


Table S4: Clusters identified using a genetic distance threshold within $1 \%$ of the distribution of patristic distances within the entire tree. The minimum percentile threshold that maximized the number of clusters was chosen as the optimal threshold by performing multiple clustering runs on randomly sampled patristic distance distributions (1 million for each run) in Phylopart v2 (46).

\begin{tabular}{|c|c|c|c|c|c|}
\hline clustername & bootstrap & leafname & branchPath & medianOfDistances & sequencesperCluster \\
\hline 4142 & 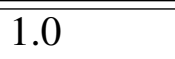 & "EPI_ISL_429609 & 6.413599999999988E-4 & 8.430999999999868E-5 & 185 \\
\hline 4142 & 1.0 & EPI_ISL_424334 & 6.539399999999993E-4 & 8.430999999999868E-5 & 185 \\
\hline 4142 & 1.0 & EPI_ISL_424274 & 7.530899999999996E-4 & 8.430999999999868E-5 & 185 \\
\hline 4142 & 1.0 & EPI_ISL_422996 & 6.151999999999993E-4 & 8.430999999999868E-5 & 185 \\
\hline 4142 & 1.0 & EPI_ISL_418073 & 6.413599999999988E-4 & 8.430999999999868E-5 & 185 \\
\hline 4142 & 1.0 & EPI_ISL_426109 & 6.477299999999995E-4 & 8.430999999999868E-5 & 185 \\
\hline 4142 & 1.0 & EPI_ISL_426085 & 6.457199999999987E-4 & 8.430999999999868E-5 & 185 \\
\hline 4142 & 1.0 & EPI_ISL_416729 & $6.282799999999991 \mathrm{E}-4$ & 8.430999999999868E-5 & 185 \\
\hline 4142 & 1.0 & EPI_ISL_418953 & 6.71379999999999E-4 & 8.430999999999868E-5 & 185 \\
\hline 4142 & 1.0 & EPI_ISL_416648 & 6.413599999999988E-4 & 8.430999999999868E-5 & 185 \\
\hline 4142 & 1.0 & EPI_ISL_416725 & 6.435399999999988E-4 & 8.430999999999868E-5 & 185 \\
\hline 4142 & 1.0 & EPI_ISL_418033 & $6.522599999999986 \mathrm{E}-4$ & 8.430999999999868E-5 & 185 \\
\hline 4511 & 1.0 & EPI_ISL_421420 & $5.737800000000002 \mathrm{E}-4$ & 7.600999999999984E-5 & 3 \\
\hline 4511 & 1.0 & EPI_ISL_429642 & $6.103400000000002 \mathrm{E}-4$ & 7.600999999999984E-5 & 3 \\
\hline 4511 & 1.0 & EPI_ISL_427165 & 6.819900000000003E-4 & 7.600999999999984E-5 & 3 \\
\hline 4518 & 1.0 & EPI_ISL_423030 & $5.759500000000001 \mathrm{E}-4$ & 4.359999999999911E-6 & 2 \\
\hline 4518 & 1.0 & EPI_ISL_427217 & $5.759500000000001 \mathrm{E}-4$ & 4.359999999999911E-6 & 2 \\
\hline 4522 & 1.0 & EPI_ISL_424186 & 7.203600000000001E-4 & 8.020999999999983E-5 & 7 \\
\hline 4522 & 1.0 & EPI_ISL_429603 & $6.5184 \mathrm{E}-4$ & 8.020999999999983E-5 & 7 \\
\hline 4522 & 1.0 & EPI_ISL_427267 & $6.840300000000001 \mathrm{E}-4$ & 8.020999999999983E-5 & 7 \\
\hline 4522 & 1.0 & EPI_ISL_427192 & $6.5184 \mathrm{E}-4$ & 8.020999999999983E-5 & 7 \\
\hline 4522 & 1.0 & EPI_ISL_424341 & $6.846 \mathrm{E}-4$ & 8.020999999999983E-5 & 7 \\
\hline 4522 & 1.0 & EPI_ISL_418954 & $6.1311 \mathrm{E}-4$ & 8.020999999999983E-5 & 7 \\
\hline 4522 & 1.0 & EPI_ISL_427620 & $6.474800000000001 \mathrm{E}-4$ & 8.020999999999983E-5 & 7 \\
\hline 4566 & 1.0 & EPI_ISL_420608 & $5.307000000000002 \mathrm{E}-4$ & 4.359999999999911E-6 & 3 \\
\hline 4566 & 1.0 & EPI_ISL_418430 & $5.307000000000002 \mathrm{E}-4$ & 4.359999999999911E-6 & 3 \\
\hline
\end{tabular}


Table S4: Clusters identified using a genetic distance threshold within $1 \%$ of the distribution of patristic distances within the entire tree. The minimum percentile threshold that maximized the number of clusters was chosen as the optimal threshold by performing multiple clustering runs on randomly sampled patristic distance distributions (1 million for each run) in Phylopart v2 (46).

\begin{tabular}{|c|c|c|c|c|c|}
\hline clustername & bootstrap & leafname & branchPath & medianOfDistances & sequencesperCluster \\
\hline $4 \overline{4566}$ & 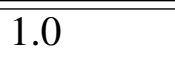 & "EPI_ISL_419173 & 5.307000000000002E-4 & 4.359999999999911E-6 & 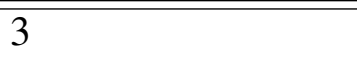 \\
\hline 4594 & 1.0 & EPI_ISL_418413 & $5.503199999999999 \mathrm{E}-4$ & 3.872999999999984E-5 & 2 \\
\hline 4594 & 1.0 & EPI_ISL_420615 & 5.846899999999999E-4 & 3.872999999999984E-5 & 2 \\
\hline 4612 & 1.0 & EPI_ISL_420617 & $5.372400000000001 \mathrm{E}-4$ & 3.881999999999996E-5 & 2 \\
\hline 4612 & 1.0 & EPI_ISL_416758 & $5.717000000000002 \mathrm{E}-4$ & 3.881999999999996E-5 & 2 \\
\hline 4625 & 1.0 & EPI_ISL_429669 & $5.759600000000001 \mathrm{E}-4$ & 3.87199999999999E-5 & 2 \\
\hline 4625 & 1.0 & EPI_ISL_424929 & $5.416 \mathrm{E}-4$ & 3.87199999999999E-5 & 2 \\
\hline 4628 & 1.0 & EPI_ISL_419172 & $5.4194 \mathrm{E}-4$ & 4.359999999999911E-6 & 2 \\
\hline 4628 & 1.0 & EPI_ISL_419171 & 5.4194E-4 & 4.359999999999911E-6 & 2 \\
\hline 4640 & 1.0 & EPI_ISL_424023 & $6.1951 \mathrm{E}-4$ & 8.617999999999972E-5 & 63 \\
\hline 4640 & 1.0 & EPI_ISL_424150 & 6.325899999999997E-4 & 8.617999999999972E-5 & 63 \\
\hline 4640 & 1.0 & EPI_ISL_423400 & 6.952999999999992E-4 & 8.617999999999972E-5 & 63 \\
\hline 4640 & 1.0 & EPI_ISL_423261 & 6.822199999999994E-4 & 8.617999999999972E-5 & 63 \\
\hline 4640 & 1.0 & EPI_ISL_418701 & 6.522099999999993E-4 & 8.617999999999972E-5 & 63 \\
\hline 4640 & 1.0 & EPI_ISL_421791 & 6.969699999999999E-4 & 8.617999999999972E-5 & 63 \\
\hline 4640 & 1.0 & EPI_ISL_423498 & 7.057099999999997E-4 & 8.617999999999972E-5 & 63 \\
\hline 4640 & 1.0 & EPI_ISL_424022 & 6.260499999999998E-4 & 8.617999999999972E-5 & 63 \\
\hline 4640 & 1.0 & EPI_ISL_424153 & 6.413099999999995E-4 & 8.617999999999972E-5 & 63 \\
\hline 4640 & 1.0 & EPI_ISL_423262 & 6.887599999999993E-4 & 8.617999999999972E-5 & 63 \\
\hline 4640 & 1.0 & EPI_ISL_423965 & 6.282299999999998E-4 & 8.617999999999972E-5 & 63 \\
\hline 4640 & 1.0 & EPI_ISL_421778 & $6.756799999999996 \mathrm{E}-4$ & 8.617999999999972E-5 & 63 \\
\hline 4640 & 1.0 & EPI_ISL_423105 & 6.522099999999993E-4 & 8.617999999999972E-5 & 63 \\
\hline 4640 & 1.0 & EPI_ISL_423966 & 6.391299999999996E-4 & 8.617999999999972E-5 & 63 \\
\hline 4640 & 1.0 & EPI_ISL_423988 & 6.238699999999999E-4 & 8.617999999999972E-5 & 63 \\
\hline 4640 & 1.0 & EPI_ISL_423733 & 7.01839999999999E-4 & 8.617999999999972E-5 & 63 \\
\hline 4640 & 1.0 & EPI_ISL_419811 & 7.193799999999995E-4 & 8.617999999999972E-5 & 63 \\
\hline
\end{tabular}


Table S4: Clusters identified using a genetic distance threshold within $1 \%$ of the distribution of patristic distances within the entire tree. The minimum percentile threshold that maximized the number of clusters was chosen as the optimal threshold by performing multiple clustering runs on randomly sampled patristic distance distributions (1 million for each run) in Phylopart v2 (46).

\begin{tabular}{|c|c|c|c|c|c|}
\hline clustername & bootstrap & leafname & branchPath & medianOfDistances & sequencesperCluster \\
\hline 4640 & 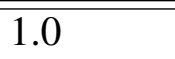 & "EPI_ISL_420724 & 6.691399999999997E-4 & $8.617999999999972 \mathrm{E}-5$ & 63 \\
\hline 4640 & 1.0 & EPI_ISL_423732 & 6.778599999999995E-4 & 8.617999999999972E-5 & 63 \\
\hline 4640 & 1.0 & EPI_ISL_421863 & 6.347699999999997E-4 & 8.617999999999972E-5 & 63 \\
\hline 4640 & 1.0 & EPI_ISL_424136 & 7.485099999999996E-4 & 8.617999999999972E-5 & 63 \\
\hline 4640 & 1.0 & EPI_ISL_423892 & 6.434899999999995E-4 & 8.617999999999972E-5 & 63 \\
\hline 4640 & 1.0 & EPI_ISL_420479 & 6.413099999999995E-4 & 8.617999999999972E-5 & 63 \\
\hline 4640 & 1.0 & EPI_ISL_423147 & 7.318499999999992E-4 & 8.617999999999972E-5 & 63 \\
\hline 4640 & 1.0 & EPI_ISL_421772 & 6.522099999999993E-4 & 8.617999999999972E-5 & 63 \\
\hline 4640 & 1.0 & EPI_ISL_420709 & 6.913499999999993E-4 & 8.617999999999972E-5 & 63 \\
\hline 4640 & 1.0 & EPI_ISL_421788 & 6.700599999999997E-4 & 8.617999999999972E-5 & 63 \\
\hline 4640 & 1.0 & EPI_ISL_416517 & 6.543899999999993E-4 & 8.617999999999972E-5 & 63 \\
\hline 4640 & 1.0 & EPI_ISL_421894 & 6.909399999999993E-4 & 8.617999999999972E-5 & 63 \\
\hline 4640 & 1.0 & EPI_ISL_421862 & 6.522099999999993E-4 & 8.617999999999972E-5 & 63 \\
\hline 4640 & 1.0 & EPI_ISL_423692 & 6.604199999999999E-4 & 8.617999999999972E-5 & 63 \\
\hline 4640 & 1.0 & EPI_ISL_421815 & 6.991499999999998E-4 & 8.617999999999972E-5 & 63 \\
\hline 4640 & 1.0 & EPI_ISL_423484 & 6.669599999999998E-4 & 8.617999999999972E-5 & 63 \\
\hline 4640 & 1.0 & EPI_ISL_423634 & 6.778599999999995E-4 & 8.617999999999972E-5 & 63 \\
\hline 4640 & 1.0 & EPI_ISL_421799 & 6.478499999999994E-4 & 8.617999999999972E-5 & 63 \\
\hline 4640 & 1.0 & EPI_ISL_421912 & 6.434899999999995E-4 & 8.617999999999972E-5 & 63 \\
\hline 4640 & 1.0 & EPI_ISL_420635 & 6.734999999999996E-4 & 8.617999999999972E-5 & 63 \\
\hline 4640 & 1.0 & EPI_ISL_425273 & 7.263099999999993E-4 & 8.617999999999972E-5 & 63 \\
\hline 4640 & 1.0 & EPI_ISL_420478 & 6.500299999999994E-4 & 8.617999999999972E-5 & 63 \\
\hline 4640 & 1.0 & EPI_ISL_424012 & 7.01839999999999E-4 & 8.617999999999972E-5 & 63 \\
\hline 4640 & 1.0 & EPI_ISL_420727 & 7.034999999999998E-4 & 8.617999999999972E-5 & 63 \\
\hline 4640 & 1.0 & EPI_ISL_421774 & 7.013299999999998E-4 & 8.617999999999972E-5 & 63 \\
\hline 4640 & 1.0 & EPI_ISL_423260 & 6.691399999999997E-4 & 8.617999999999972E-5 & 63 \\
\hline
\end{tabular}


Table S4: Clusters identified using a genetic distance threshold within $1 \%$ of the distribution of patristic distances within the entire tree. The minimum percentile threshold that maximized the number of clusters was chosen as the optimal threshold by performing multiple clustering runs on randomly sampled patristic distance distributions (1 million for each run) in Phylopart v2 (46).

\begin{tabular}{|c|c|c|c|c|c|}
\hline clustername & bootstrap & leafname & branchPath & medianOfDistances & sequencesperCluster \\
\hline 4640 & 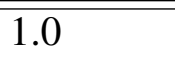 & "EPI_ISL_421895 & 6.843999999999994E-4 & 8.617999999999972E-5 & $\overline{63}$ \\
\hline 4640 & 1.0 & EPI_ISL_421775 & 6.947899999999999E-4 & 8.617999999999972E-5 & 63 \\
\hline 4640 & 1.0 & EPI_ISL_421779 & 7.01839999999999E-4 & 8.617999999999972E-5 & 63 \\
\hline 4640 & 1.0 & EPI_ISL_423296 & 6.711999999999997E-4 & 8.617999999999972E-5 & 63 \\
\hline 4640 & 1.0 & EPI_ISL_423987 & 6.745599999999997E-4 & 8.617999999999972E-5 & 63 \\
\hline 4640 & 1.0 & EPI_ISL_423446 & 6.369499999999996E-4 & 8.617999999999972E-5 & 63 \\
\hline 4640 & 1.0 & EPI_ISL_423265 & 6.800399999999995E-4 & 8.617999999999972E-5 & 63 \\
\hline 4640 & 1.0 & EPI_ISL_419998 & 7.215799999999994E-4 & 8.617999999999972E-5 & 63 \\
\hline 4640 & 1.0 & EPI_ISL_425336 & 7.162699999999995E-4 & 8.617999999999972E-5 & 63 \\
\hline 4640 & 1.0 & EPI_ISL_423693 & 6.999799999999998E-4 & 8.617999999999972E-5 & 63 \\
\hline 4640 & 1.0 & EPI_ISL_423264 & 6.931199999999992E-4 & 8.617999999999972E-5 & 63 \\
\hline 4640 & 1.0 & EPI_ISL_418756 & 6.522099999999993E-4 & 8.617999999999972E-5 & 63 \\
\hline 4640 & 1.0 & EPI_ISL_421798 & 6.778599999999995E-4 & 8.617999999999972E-5 & 63 \\
\hline 4640 & 1.0 & EPI_ISL_423453 & 6.865899999999994E-4 & 8.617999999999972E-5 & 63 \\
\hline 4640 & 1.0 & EPI_ISL_421796 & 7.013299999999998E-4 & 8.617999999999972E-5 & 63 \\
\hline 4640 & 1.0 & EPI_ISL_423256 & 6.865799999999994E-4 & 8.617999999999972E-5 & 63 \\
\hline 4640 & 1.0 & EPI_ISL_428930 & 6.522099999999993E-4 & 8.617999999999972E-5 & 63 \\
\hline 4640 & 1.0 & EPI_ISL_421819 & 6.369499999999996E-4 & 8.617999999999972E-5 & 63 \\
\hline 4640 & 1.0 & EPI_ISL_423741 & 6.996599999999991E-4 & 8.617999999999972E-5 & 63 \\
\hline 4640 & 1.0 & EPI_ISL_423691 & 6.625999999999998E-4 & 8.617999999999972E-5 & 63 \\
\hline 4768 & 1.0 & EPI_ISL_423394 & 6.990699999999999E-4 & 6.539999999999975E-6 & 3 \\
\hline 4768 & 1.0 & EPI_ISL_423399 & 6.990699999999999E-4 & 6.539999999999975E-6 & 3 \\
\hline 4768 & 1.0 & EPI_ISL_423276 & 6.968899999999999E-4 & 6.539999999999975E-6 & 3 \\
\hline 4783 & 1.0 & EPI_ISL_430066 & 7.034199999999997E-4 & 6.539999999999758E-6 & 4 \\
\hline 4783 & 1.0 & EPI_ISL_427081 & 7.034199999999997E-4 & 6.539999999999758E-6 & 4 \\
\hline 4783 & 1.0 & EPI_ISL_420876 & 7.034199999999997E-4 & 6.539999999999758E-6 & 4 \\
\hline
\end{tabular}


Table S4: Clusters identified using a genetic distance threshold within $1 \%$ of the distribution of patristic distances within the entire tree. The minimum percentile threshold that maximized the number of clusters was chosen as the optimal threshold by performing multiple clustering runs on randomly sampled patristic distance distributions (1 million for each run) in Phylopart v2 (46).

\begin{tabular}{|c|c|c|c|c|c|}
\hline clustername & bootstrap & leafname & branchPath & medianOfDistances & sequencesperCluster \\
\hline 4783 & 1.0 & EPI_ISL_427088 & 7.012399999999998E-4 & 6.539999999999758E-6 & 4 \\
\hline 4791 & 1.0 & EPI_ISL_420045 & $6.8818 \mathrm{E}-4$ & $7.312000000000009 \mathrm{E}-5$ & 2 \\
\hline 4791 & 1.0 & EPI_ISL_428358 & $6.8818 \mathrm{E}-4$ & 7.312000000000009E-5 & 2 \\
\hline 4794 & 1.0 & EPI_ISL_420034 & $5.7861 \mathrm{E}-4$ & 6.539999999999975E-6 & 3 \\
\hline 4794 & 1.0 & EPI_ISL_420839 & $5.8079 \mathrm{E}-4$ & 6.539999999999975E-6 & 3 \\
\hline 4794 & 1.0 & EPI_ISL_417440 & $5.8079 \mathrm{E}-4$ & 6.539999999999975E-6 & 3 \\
\hline 4803 & 1.0 & EPI_ISL_419929 & 6.282299999999998E-4 & 4.582999999999996E-5 & 15 \\
\hline 4803 & 1.0 & EPI_ISL_419177 & 6.330399999999998E-4 & 4.582999999999996E-5 & 15 \\
\hline 4803 & 1.0 & EPI_ISL_428712 & 6.309699999999998E-4 & 4.582999999999996E-5 & 15 \\
\hline 4803 & 1.0 & EPI_ISL_423345 & 5.851399999999999E-4 & 4.582999999999996E-5 & 15 \\
\hline 4803 & 1.0 & EPI_ISL_428962 & $6.264399999999998 \mathrm{E}-4$ & 4.582999999999996E-5 & 15 \\
\hline 4803 & 1.0 & EPI_ISL_420606 & 6.025799999999996E-4 & 4.582999999999996E-5 & 15 \\
\hline
\end{tabular}

